\title{
THE INTERSECTION MOTIVE OF THE MODULI STACK OF SHTUKAS
}

\author{
TIMO RICHARZ ${ }^{1}$ and JAKOB SCHOLBACH ${ }^{\circledR 2}$ \\ ${ }^{1}$ TU Darmstadt, Germany; \\ email: richarz@mathematik.tu-darmstadt.de \\ ${ }^{2}$ Universität Münster, Germany; \\ email: jakob.scholbach@uni-muenster.de
}

Received 17 January 2019; accepted 29 August 2019

\begin{abstract}
For a split reductive group $G$ over a finite field, we show that the intersection (cohomology) motive of the moduli stack of iterated $G$-shtukas with bounded modification and level structure is defined independently of the standard conjectures on motivic $t$-structures on triangulated categories of motives. This is in accordance with general expectations on the independence of $\ell$ in the Langlands correspondence for function fields.
\end{abstract}

2010 Mathematics Subject Classification: 20G05 (primary); 14M15, 19E15 (secondary)

\section{Introduction}

1.1. Motivation and goals. Let $X$ be a variety over a field $k$, and fix a prime number $\ell \in \mathbf{Z}$ invertible on $k$. Since Grothendieck's construction of the $\ell$-adic étale cohomology groups $\mathrm{H}_{\mathrm{et}}^{i}\left(X, \mathbf{Q}_{\ell}\right)$, a central question is whether these are independent of the auxiliary prime number $\ell$, or even whether it is possible to find 'natural' rational structures on them. This leads to the theory of motives. Envisioned by Beilinson, and realized by Voevodsky [Voe00], Levine [Lev98], and Hanamura [Han95] for motives over $S=\operatorname{Spec}(k)$, and extended by Ayoub [Ayo07a, Ayo07b, Ayo14] and Cisinski and Déglise [CD09, CD16] to motives over general base schemes $S$, there now exists a theory of motivic sheaves, that is, a full six-functor formalism for suitable triangulated categories $\operatorname{DM}(S)=\operatorname{DM}(S, \mathbf{Q})$ of motives with rational coefficients over $S$. By construction this theory of motives is independent of $\ell$, but explicit computations are difficult.

(c) The Author(s) 2020. This is an Open Access article, distributed under the terms of the Creative Commons Attribution licence (http://creativecommons.org/licenses/by/4.0/), which permits unrestricted re-use, distribution, and reproduction in any medium, provided the original work is properly cited. 
One of the main obstacles is the lack of a motivic $t$-structure on these categories, that is, the existence of an object $\mathrm{h}^{i}(X) \in \mathrm{DM}(\operatorname{Spec} k)$ whose $\ell$-adic realization would be $\mathrm{H}_{\mathrm{et}}^{i}\left(X, \mathbf{Q}_{\ell}\right)$. This is part of the package of standard conjectures on motives which seem to be out of reach at the moment.

However, Levine [Lev93] showed that a $t$-structure does exist on the subcategory $\operatorname{DTM}(S) \subset \operatorname{DM}(S)$ of Tate motives for certain 'nice' $S$, for example, $S=\operatorname{Spec}\left(\mathbf{F}_{q}\right)$ or a smooth curve over $\mathbf{F}_{q}$, cf. Example 3.2.3. By definition, $\operatorname{DTM}(S)$ is the subcategory generated by motives of $\mathbf{P}_{S}^{n}(n \geqslant 0)$ and their duals. Soergel and Wendt [SW18] have extended Levine's observation to the case when $X$ is an $S$-scheme equipped with a so-called cellular Whitney-Tate stratification: loosely speaking, this condition means that the strata of $X$ are built out of products of $\mathbf{G}_{m, S}$ or $\mathbf{A}_{S}^{1}$, and that one needs to be able to control the singularities of the closures of the strata. While this condition is rather restrictive in general, it turns out that several varieties $X$ of interest in geometric representation theory do carry such stratifications. For example, the flag variety $X=G / B$ associated with a split reductive $k$-group $G$ and a Borel subgroup $B$, equipped with its stratification by $B$-orbits, has this property [SW18, Proposition 4.10]. In this situation, the category DTM $(X)$ of stratified Tate motives, that is, those which are Tate motives on each stratum, carries a $t$-structure whose heart is the abelian category of mixed stratified Tate motives $\operatorname{MTM}(X)$. The simple objects in $\operatorname{MTM}(X)$ are Tate twists of the intersection motives on the closures of the orbits of the left $B$-action on $X$.

The present paper is the first in a series aiming toward systematically applying the theory of motives as above to the constructions in the work of Lafforgue [Laf18] on the Langlands correspondence over function fields. Here, we have two goals:

(i) We provide a framework to handle motives on a large class of 'geometric objects', namely prestacks.

(ii) We show that the intersection cohomology motive of the moduli stack of iterated $G$-shtukas with bounded modification and level structure is unconditionally defined, that is, without reference to the standard conjectures.

Part (i) follows ideas of Gaitsgory and Rozenblyum [GR17, Section 3, 0.1.1], and of Raskin [Ras]. A benefit of Definition 2.2.1 is that categories of motives on objects such as $X=L^{+} G \backslash L G / L^{+} G$ (the double quotient of the loop group by the positive loop group) are well-defined independently of choices of presentations of the ind-scheme $L G$ or the pro-algebraic group $L^{+} G$. Here we provide only as much of the general theory as needed in order to construct (ii).

Given (i), the construction in (ii) ultimately boils down (cf. Sections 5, 6) to an extension of the methods of [SW18] and of Soergel et al. [SVW18] from 
ordinary schemes with an action of an algebraic group to ind-schemes with an action of a pro-algebraic group. Our constructions are also applied in forthcoming work [RS19] to construct a motivic Satake equivalence in this set-up.

We note that (ii) is in accordance with Lafforgue's conjecture [Laf18, Conjecture 12.12] which says that the decomposition of the space of cusp forms obtained there over $\overline{\mathbf{Q}}_{\ell}$ is in fact defined over $\overline{\mathbf{Q}}$ and does neither depend on the embedding $\overline{\mathbf{Q}} \hookrightarrow \overline{\mathbf{Q}}_{\ell}$ nor on the chosen prime number $\ell$.

1.2. Statement of results. Let $S$ be the spectrum of a field, or the spectrum of the integers (or more generally as in Notation 2.0.1). Let $\mathrm{AffSch}_{S}$ (respectively $\mathrm{AffSch}_{S}^{\mathrm{ft}}$ ) be the category of affine schemes equipped with a map (respectively finite type map) to $S$. For each $X \in \operatorname{AffSch}_{S}^{\mathrm{ft}}$, we let $\operatorname{DM}(X)=\operatorname{DM}(X, \mathbf{Q})$ be the triangulated category of motives with rational coefficients à la Ayoub and Cisinski-Déglise, cf. Section 2.1. For a map $f: X \rightarrow Y$ in $\operatorname{AffSch}_{S}^{\mathrm{ft}}$, there are pairs of adjoint functors $\left(f^{*}, f_{*}\right),\left(f_{!}, f^{!}\right)$ satisfying the usual compatibilities, cf. Synopsis 2.1.1. For our purposes, it is convenient to view $\operatorname{DM}(X)$ as a presentable stable $\infty$-category, and following Hoyois and Khan the pair of adjoint functors $\left(f^{*}, f_{*}\right),\left(f_{!}, f^{!}\right)$can be viewed as colimit-preserving functors between these categories. We need to consider motives on quite general geometric objects, for example ind-Artin stacks, or stacky quotients by pro-algebraic groups. Following ideas of GaitsgoryRozenblyum and Raskin, it is convenient to use the notion of $\infty$-prestacks: The category of prestacks is the functor category in the sense of Lurie

$$
\operatorname{PreStk}_{S} \stackrel{\text { def }}{=} \operatorname{Fun}\left(\left(\mathrm{AffSch}_{S}\right)^{\mathrm{op}}, \infty-\mathrm{Gpd}\right) .
$$

The existence of all (homotopy) limits and colimits in $\operatorname{PreStk}_{S}$ has the advantage that ind-objects (ind-schemes, ind-Artin stacks), pro-objects (pro-algebraic groups), or quotients under group actions are prestacks.

Theorem A. (i) The presheaf of $\infty$-categories DM: $\left(\mathrm{AffSch}_{S}^{\mathrm{ft}}\right)^{\mathrm{op}} \rightarrow \mathrm{Cat}_{\infty}$ given by

$$
X \mapsto \operatorname{DM}(X), \quad f \mapsto f^{!}
$$

can be upgraded to a presheaf $\mathrm{DM}:$ PreStk $_{S} \rightarrow$ DGCat $_{\text {cont }}$ where the target is the $\infty$-category of presentable stable $\mathbf{Q}$-linear $d g-\infty$-categories with continuous functors. In particular, for any map of prestacks $f: X \rightarrow Y$ there is a functor $f^{!}: \mathrm{DM}(Y) \rightarrow \operatorname{DM}(X)$ in $\mathrm{DGCat}_{\text {cont }}$ (see Section 2.2).

(ii) The presheaf $\mathrm{DM}: \operatorname{PreStk}_{S} \rightarrow \mathrm{DGCat}_{\text {cont }}$ is a sheaf of $\infty$-categories for Voevodsky's h-topology (see 2.1.13, 2.1.14, 2.2.16). 
(iii) For any map $f: X \rightarrow Y$ of strict ind-Artin stacks ind-(locally of finite type) over $S$, there is an adjunction

$$
f_{!}: \mathrm{DM}(X) \leftrightarrows \mathrm{DM}(Y): f^{!}
$$

For any prime $\ell \in \mathbf{Z}$ invertible on $S$, this adjunction agrees under the $\ell$-adic étale realization functor with the adjunction constructed in the work of Liu-Zheng in the case of Artin stacks $X$ and $Y$ [LZ11, LZ14] (see 2.3.7).

(iv) For strict ind-schemes of ind-(finite type) over $S$, there is a six-functor formalism under certain restrictions on the *-pullback functor (see 2.4.2).

(v) Hom-sets in the category $\operatorname{DM}(X / G)$ of equivariant motives reproduce equivariant higher Chow groups as introduced by Totaro et al. [Tot99, Tot16, EG98] (see 2.2.10).

We comment on the result: in (i), it is also possible to upgrade the presheaf $X \mapsto \operatorname{DM}(X), f \mapsto f^{*}$ to prestacks. We work with the !-pullback because of the following result of Lurie (cf. Lemma 2.3.2 below): Let $X=\operatorname{colim}_{i} X_{i}$ where $X_{i}$ are Artin stacks locally of finite type over $S$, and the transition maps $t_{i, j}: X_{i} \rightarrow X_{j}$ are closed immersions. Then there are natural equivalences (in DGCat ${ }_{\text {cont }}$ )

$$
\operatorname{DM}(X)=\lim _{t^{!}} \operatorname{DM}\left(X_{i}\right)=\underset{t_{!}}{\operatorname{colim}} \operatorname{DM}\left(X_{i}\right) .
$$

This is in accordance with ad hoc definitions of say bounded derived categories of étale constructible sheaves on strict ind-Artin stacks or strict ind-schemes as commonly used. Part (ii) shows that the category of motives on prestacks is insensitive to $\tau$-stackification (in the $\infty$-sense) where $\tau$ is a Grothendieck topology contained in the $h$-topology. More precisely, for any prestack $X$, the !-pullback along the canonical map $X \rightarrow X^{\tau}$ induces an equivalence (in DGCat $_{\text {cont }}$ )

$$
\operatorname{DM}\left(X^{\tau}\right)=\operatorname{DM}(X) .
$$

In particular (take $\tau=$ Zar), it shows that for all Noetherian schemes of finite Krull dimension $X$ (equipped with a map to $S$ ) the category $\operatorname{DM}(X)$ is the category of motives as defined in Cisinski and Déglise [CD09], cf. Remark 2.2.2(v). Part (ii) is similar to [Hoy17, Proposition 6.23]. Part (iii) allows to conveniently define the motive of an ind-Artin stack $f: X \rightarrow S$ ind-(locally of finite type) as

$$
M(X) \stackrel{\text { def }}{=} f_{!} f^{!}(X) \in \mathrm{DM}(S) .
$$

This recovers the motive $\mathrm{M}(X)$ of finite type schemes $X$ over a perfect field defined by Voevodsky, and puts computations of the motive of the affine 
Grassmannian in [Bac19], or of the motive of the moduli stack of vector bundles on a curve in [HL18] into a more functorial context. Part (iv) concludes the basic framework as needed in the constructions of the present manuscript. To keep the manuscript at a reasonable length, we chose not to discuss extensions of the full six-functor formalism, say to (higher) Artin stacks as provided in the étale set-up by [LZ11, LZ14].

With applications to geometric representation theory in mind, and more specifically to [Laf18], we aim to construct intersection motives. Their existence is predicted by the standard conjectures. In order to make our results unconditional, we need to drastically restrict the class of objects, cf. Section 3. Here, we consider a strict ind-scheme of ind-finite type over $S$ equipped with a stratification into locally closed strata

$$
\iota: X^{+}:=\bigsqcup_{w \in W} X_{w} \rightarrow X,
$$

where each stratum $X_{w}$ is a cellular $S$-scheme, that is, each $X_{w} \rightarrow S$ is smooth, and can be stratified further by products of $\mathbf{G}_{m, S}$ or $\mathbf{A}_{S}^{1}$. Let $\operatorname{DTM}\left(X^{+}\right) \subset \operatorname{DM}\left(X^{+}\right)$ be the full subcategory generated by Tate motives, that is, by all unit motives $1_{X_{w}}(n)[m]$, for all $w \in W, n, m \in \mathbf{Z}$, cf. Definition 3.1.8. Following Soergel and Wendt [SW18, Definition 4.5] (who consider the case of finite type schemes over fields), the stratification $\iota: X^{+} \rightarrow X$ is called Whitney-Tate if

$$
\iota^{*} \iota_{*} 1_{X^{+}} \in \operatorname{DTM}\left(X^{+}\right),
$$

that is, $\left(\left.\iota\right|_{X_{v}}\right)^{*}\left(\left.\iota\right|_{X_{w}}\right)_{*} 1_{X_{w}}$ is a Tate motive on $X_{v}$ for each $v, w \in W$. As is well known [BBD82, 1.4.9], this condition ensures that the categories of Tate motives on the single strata can be glued together. Applications of Whitney-Tate stratified motives to representation theory have also been studied by Eberhardt and Kelly [EK19].

THEOREM B. Let $X$ be an ind-scheme of ind-finite type over $S$ equipped with a cellular Whitney-Tate stratification $\iota: X^{+} \rightarrow X$.

(i) There is a well-defined stable full $\infty$-subcategory $\operatorname{DTM}(X) \subset \operatorname{DM}(X)$ of stratified Tate motives. It consists of those motives $M$ such that the pullback $\iota^{*} M$ or, equivalently $\iota^{!} M$ is Tate, that is, lies in $\operatorname{DTM}\left(X^{+}\right)$(see 3.1.11).

(ii) If $S$ satisfies the Beilinson-Soulé vanishing conjecture, there is a self-dual motivic $t$-structure on $\operatorname{DTM}(X)$. Its heart $\operatorname{MTM}(X)$ is generated by the intersection motives

$$
\mathrm{IC}_{w}(n) \stackrel{\text { def }}{=} j_{w, ! *}\left(1_{X_{w}}(n)\left[d_{w}\right]\right) \in \operatorname{DTM}(X)
$$


supported on the closure $\bar{X}_{w}$, where $j_{w}: X_{w} \rightarrow \bar{X}_{w}, w \in W, n \in \mathbf{Z}$. These are precisely the simple objects in $\operatorname{MTM}(X)$, and their $\ell$-adic realizations are the intersection complexes defined by the middle perverse extension of the constant $\ell$-adic sheaves $\mathbf{Q}_{\ell}(n)$ on the strata $X_{w}$ (see 3.2.6, 3.3.8).

(iii) Let $G=\lim _{i \geqslant 0} G_{i}$ be a pro-smooth affine $S$-group scheme acting on $X$ compatibly with the stratification. Assume that the scheme underlying each $G_{i}$ is cellular, and that each $\operatorname{ker}\left(G_{i+1} \rightarrow G_{i}\right)$ is a vector group. There is a well-defined stable full $\infty$-subcategory $\operatorname{DTM}_{G}(X) \subset \mathrm{DM}(G \backslash X)$ of equivariant stratified Tate motives where $G \backslash X$ is the prestack quotient. If $S$ is as in (ii), then there is a self-dual motivic $t$-structure on $\operatorname{DTM}_{G}(X)$. Its heart $\mathrm{MTM}_{G}(X)$ contains the intersection motives $\mathrm{IC}_{w}(n)$ which map under the forgetful functor

$$
\operatorname{MTM}_{G}(X) \rightarrow \operatorname{MTM}(X)
$$

to the intersection motives as in (ii). If $G$ has connected fibers, this forgetful functor is fully faithful. If in addition the stabilizers of the G-action are connected, then $\operatorname{MTM}_{G}(X)$ is also generated by the $\operatorname{IC}_{w}(n), w \in W, n \in \mathbf{Z}$ (see 3.1.21, 3.1.27, 3.2.15, 3.2.20, 3.2.23).

Having the six-functor formalism for motives on ind-schemes, the proofs of (i) and (ii) are immediate from [SW18]. We note that the Beilinson-Soulé conjecture is known by Quillen's, Borel's, and Harder's work, for $S$ being the spectrum of a finite field, a number field or localizations of its ring of integers, for a smooth curve over a finite or its function field, and finally for filtered colimits of such objects, cf. Example 3.2.3. We now comment on (iii). The notation $\operatorname{DTM}_{G}(X)$ (as opposed to $\operatorname{DTM}(G \backslash X))$ highlights the fact that this category depends on the chosen presentation of the prestack $G \backslash X$. Put differently, Tate motives do (by definition) not satisfy descent, cf. Example 3.1.25. The assumption on $\operatorname{ker}\left(G_{i+1} \rightarrow G_{i}\right)$ being a vector group ensures that $\operatorname{DM}\left(G \backslash \bar{X}_{w}\right)=\operatorname{DM}\left(G_{i} \backslash \bar{X}_{w}\right)$ for $i \gg 0$. It is satisfied for an interesting class of pro-smooth affine group schemes which are constructed as 'positive loop groups' (a.k.a. 'jet groups'), cf. Proposition A.9.

We now give two closely related applications of the theory developed so far, cf. Sections 5-6. Let $G$ be a split reductive group over the integers $\mathbf{Z}$ (a.k.a. a Chevalley group), and fix a Borel pair $T \subset B$. The loop group is the functor

$$
L G: \text { Rings } \rightarrow \text { Grps, } \quad R \mapsto G(R((\varpi))),
$$

where $R((\varpi))$ denotes the Laurent series ring in the formal variable $\varpi$. The group functor $L G$ is represented by an ind-affine ind-scheme over $\mathbf{Z}$. Associated with each facet $\mathbf{f}$ in the standard apartment $\mathscr{A}=\mathscr{A}(G, T)$ of the Bruhat-Tits building 
is the closed $\mathbf{Z}$-subgroup

$$
\mathcal{P}_{\mathbf{f}} \subset L G,
$$

called parahoric subgroup. The group $\mathcal{P}_{\mathbf{f}}=\lim _{i \geqslant 0} \mathcal{P}_{\mathbf{f}, i}$ is a pro-smooth affine $\mathbf{Z}$-group scheme with connected fibers such that each $\operatorname{ker}\left(\mathcal{P}_{\mathbf{f}, i+1} \rightarrow \mathcal{P}_{\mathbf{f}, i}\right)$ is a vector group. If $\mathbf{f}=\{0\} \subset \mathscr{A}$ is the base point, then $L^{+} G=\mathcal{P}_{\{0\}}$ is the positive loop group given by $L^{+} G(R)=G(R \llbracket \varpi \rrbracket)$. If $\mathbf{f}=\mathbf{a}_{0}$ is the standard alcove determined by $B$, then $\mathcal{P}_{\mathbf{a}_{0}}$ is the (standard) Iwahori subgroup given as the preimage of $B$ under the reduction map $L^{+} G \rightarrow G, \varpi \mapsto 0$. For general facets, these groups can be described in terms of Bruhat-Tits theory.

Now fix two facets $\mathbf{f}, \mathbf{f}^{\prime} \subset \mathscr{A}$ which are contained in the closure of the standard alcove. We are interested in motives on the double quotient

$$
\mathcal{P}_{\mathbf{f}^{\prime}} \backslash L G / \mathcal{P}_{\mathbf{f}} \in \operatorname{PreStk}_{\mathrm{Spec}(\mathbf{Z})} .
$$

We recover the set-up of Theorem $B$ by considering the left- $\mathcal{P}_{\mathbf{f}^{\prime}}$-action on the ind-

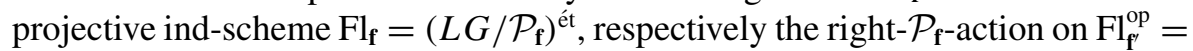

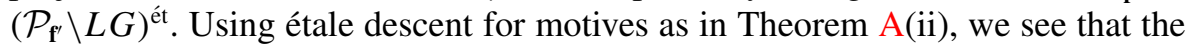
resulting equivariant categories are the same, and equal to $\operatorname{DM}\left(\mathcal{P}_{\mathbf{f}^{\prime}} \backslash L G / \mathcal{P}_{\mathbf{f}}\right)$. For convenience of the reader, we note that the étale stackification $\left(\mathcal{P}_{\mathbf{f}^{\prime}} \backslash L G / \mathcal{P}_{\mathbf{f}}\right)^{\text {et }}$ automatically is an fpqc stack, cf. Lemma 5.3.2.

THEOREM C. (i) The stratification of $\mathrm{Fl}_{\mathbf{f}}\left(\right.$ respectively $\left.\mathrm{Fl}_{\mathbf{f}^{\prime}}^{\mathrm{op}}\right)$ by orbits of the left-

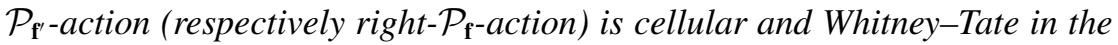
sense outlined above. Further, Theorem $\mathrm{B}(\mathrm{i})$-(iii) applies (see 5.1.1, 5.2.3).

(ii) There is an equivalence of the resulting categories of equivariant stratified Tate motives

$$
\operatorname{DTM}_{\mathcal{P}_{\mathbf{f}^{\prime}}}\left(\mathrm{Fl}_{\mathbf{f}}\right)=\operatorname{DTM}_{\mathcal{P}_{\mathbf{f}}}\left(\mathrm{Fl}_{\mathbf{f}^{\prime}}^{\mathrm{op}}\right)
$$

as full subcategories of $\operatorname{DM}\left(\mathcal{P}_{\mathbf{f}^{\prime}} \backslash L G / \mathcal{P}_{\mathbf{f}}\right)$. If $\mathbf{f}=\mathbf{f}^{\prime}$, then the $t$-structures delivered by part (i) (and Theorem $\mathrm{B}$ ) also agree, and their heart is the Q-linear abelian full subcategory

$$
\operatorname{MTM}\left(\mathcal{P}_{\mathbf{f}} \backslash L G / \mathcal{P}_{\mathbf{f}}\right) \subset \operatorname{DM}\left(\mathcal{P}_{\mathbf{f}} \backslash L G / \mathcal{P}_{\mathbf{f}}\right),
$$

which is the symmetric version of Theorem $\mathrm{B}(\mathrm{iii})$. It is generated by intersection motives of the Schubert varieties inside $\mathrm{Fl}_{\mathbf{f}}$ (see 5.3.4).

The basic geometric properties of affine flag varieties for Chevalley groups over $\mathbf{Z}$, respectively more general base schemes $S$ are given in Section 4 . In Theorem C, we can replace $\operatorname{Spec}(\mathbf{Z})$ by any regular base scheme $S$ which satisfies the Beilinson-Soulé vanishing conjecture as above. Also it seems possible to extend our results to twisted affine flag varieties in the sense of Pappas and 
Rapoport [PR08]. In [RS19], we take $\mathbf{f}=\mathbf{f}^{\prime}=\{0\}$ and use the category $\operatorname{MTM}\left(L^{+} G \backslash L G / L^{+} G\right)$ to establish a motivic Satake equivalence in this setup. A nice feature of the symmetry of the double quotient is that the operation $L G \rightarrow L G, g \mapsto g^{-1}$ induces an anti-involution on the category of stratified mixed Tate motives.

A version of Theorem $\mathrm{C}$ for Witt vector (or $p$-adic) affine flag varieties is contained in the first arxiv version of this paper, and will be published elsewhere together with a motivic Satake equivalence in this context.

With this general set-up in hand, we construct in Section 6 the intersection cohomology motive of the moduli stack of bounded shtukas. We view these constructions as a first step toward a motivic rendition of V. Lafforgue's work in the function field case of the Langlands program since it offers a geometric understanding of the $\ell$-adic intersection cohomology of the moduli stack of $G$-shtukas in a way which is independent of $\ell$. Further steps toward this goal, including the aforementioned Satake equivalence, a motivic Drinfeld lemma, a motivic construction of excursion operators, and their identification with Hecke operators, remain to be done.

Let $T \subset B \subset G$ be defined over the finite field $k=\mathbf{F}_{q}$. Let $X$ be a smooth projective geometrically connected curve over $k$. For any effective divisor $N \subset X$ and any partitioned finite index set $I=I_{1} \sqcup \cdots \sqcup I_{r}$, there is the moduli stack of iterated $G$-shtukas $\operatorname{Sht}_{N, I}^{\left(I_{1}, \ldots, I_{r}\right)}$ with level- $N$-structure

$$
\left\langle\left(\mathcal{E}_{r}, \beta_{r}\right) \underset{I_{r}}{\stackrel{\alpha_{r}}{\rightarrow}}\left(\mathcal{E}_{r-1}, \beta_{r-1}\right) \underset{I_{r-1}}{\stackrel{\alpha_{r}}{I_{-1}}} \cdots \underset{I_{2}}{\stackrel{\alpha_{2}}{\rightarrow}}\left(\mathcal{E}_{1}, \beta_{1}\right) \underset{I_{1}}{\stackrel{\alpha_{1}}{\rightarrow}}\left(\mathcal{E}_{0}, \alpha_{0}\right)=\left({ }^{\tau} \mathcal{E}_{r},{ }^{\tau} \alpha_{r}\right)\right\rangle,
$$

as considered in [Laf18, Définition 2.1], cf. Section 6.3 for notation. This moduli stack is equipped with a forgetful map $\operatorname{Sht}_{N, I}^{\left(I_{1}, \ldots, I_{r}\right)} \rightarrow(X \backslash N)^{I}$. For an admissible tuple $\underline{\mu}=\left(\mu_{i}\right)_{i \in I} \in X_{*}(T)_{+}^{I}$ of dominant cocharacters, we can bound the relative positions of each modification $\alpha_{j}$ by $\mu_{j}$ to get a closed substack $\operatorname{Sht}_{N, I, \underline{\mu}}^{\left(I_{1}, \ldots, I_{r}\right)} \subset$ $\mathrm{Sht}_{N, I}^{\left(I_{1}, \ldots, I_{r}\right)}$ which is representable by a (reduced) Deligne-Mumford stack locally of finite type over $k$. Varying $\underline{\mu}$, we see that $\operatorname{Sht}_{N, I}^{\left(I_{1}, \ldots, I_{r}\right)}$ has the structure of a strict ind-Deligne-Mumford stack ind-(locally of finite type) over $k$. In particular, Theorem A(iii) applies in this context. Fixing a total order on $I=\{1, \ldots, n\}$ which refines the partition $I=I_{1} \sqcup \cdots \sqcup I_{r}$, there are maps of étale sheaves of groupoids

$$
\operatorname{Sht}_{N, I}^{\left(I_{1}, \ldots, I_{r}\right)} \stackrel{\pi}{\longleftarrow} \operatorname{Sht}_{N, I}^{(\{1\}, \ldots,\{n\})} \stackrel{\mathrm{inv}}{\longrightarrow} \prod_{i=1, \ldots, n}\left(L_{\{i\}}^{+} G \backslash L_{\{i\}} G / L_{\{i\}}^{+} G\right)^{\mathrm{ét}},
$$

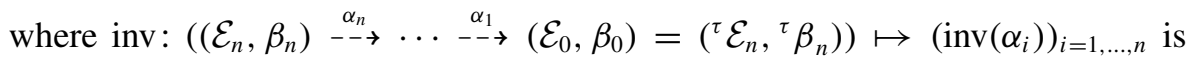
the relative position, $\mathrm{cf}$. (6.2.1). The target of the map inv is the stack of relative 
positions: each factor in the product has a forgetful map to $X$ whose fiber over $x \in X$ is $\left(L^{+} G \backslash L G / L^{+} G\right)^{\text {ét }} \bigotimes_{k} \kappa(x)$. Colloquially speaking, two such $G$-bundles $\mathcal{E}_{i}$ and $\mathcal{E}_{i-1}$ differ by an elementary modification $\alpha_{i}$ at some point of $X$, and the invariant $\operatorname{inv}\left(\alpha_{i}\right)$ is simply the double coset of the matrix changing the transition function from the one $G$-bundle to the other. Further, there is a canonical map

$$
\left(L_{\{i\}}^{+} G \backslash L_{\{i\}} G / L_{\{i\}}^{+} G\right)^{\text {ét }} \rightarrow\left(L^{+} \mathbf{G}_{m} \backslash\left(L^{+} G \backslash L G / L^{+} G\right)\right)^{\text {ét }},
$$

where $L^{+} \mathbf{G}_{m}$ acts on $L^{+} G \backslash L G / L^{+} G$ by changing the variable $\varpi$ used to form the loop groups. For each $\mu \in X_{*}(T)_{+}$, the intersection motive $\mathrm{IC}_{\mu} \in$ $\operatorname{MTM}\left(L^{+} G \backslash L G / L^{+} G\right)$ is $L^{+} \mathbf{G}_{m}$-equivariant, and its pullback via (1.2.1) is denoted $\mathrm{IC}_{\mu,\{i\}} \in \mathrm{DM}\left(\left(L_{\{i\}}^{+} G \backslash L_{\{i\}} G / L_{\{i\}}^{+} G\right)^{\text {ét }}\right)$. For each effective divisor $N \subset X$, and each admissible tuple $\underline{\mu}=\left(\mu_{i}\right)_{i \in I} \in X_{*}(T)_{+}$, we define

$$
\mathcal{F}_{N, I, \underline{\mu}}^{\left(I_{1}, \ldots, I_{r}\right)} \stackrel{\text { def }}{=} \pi_{!}\left(\operatorname{inv}^{!}\left(\bigotimes_{i=1}^{n} \mathrm{IC}_{\{i\}, \mu_{i}}\right)\right) \in \operatorname{DM}\left(\operatorname{Sht}_{N, I}^{\left(I_{1}, \ldots, I_{r}\right)}\right) .
$$

Note that the construction combines the intersection motives obtained by Theorem B (whose assumptions are satisfied by Theorem C) and the general functoriality given by Theorem A.

THEOREM D (See 6.3.5). The motive $\mathcal{F}_{N, I, \mu}^{\left(I_{1}, \ldots, I_{r}\right)}$ is supported on $\mathrm{Sht}_{N, I, \mu}^{\left(I_{1}, \ldots, I_{r}\right)}$, and its $\ell$-adic realization is (up to twist and the choice of a lattice in the adelic center) the intersection complex defined in [Laf18, Définition 2.14]. This defines the intersection cohomology motive

$$
\mathcal{H}_{N, I, \underline{\mu}} \stackrel{\text { def }}{=} p_{!}\left(\mathcal{F}_{N, I, \underline{\mu}}^{\left(I_{1}, \ldots, I_{r}\right)}\right) \in \operatorname{DM}\left((X \backslash N)^{I}\right),
$$

whose $\ell$-adic realization is (up to the normalizations above) the intersection cohomology complex defined in [Laf18, Définition 4.1].

The proof is immediate from results of Varshavsky. Namely, [Var04, Corollary 2.21] implies that the motives $\mathcal{F}_{N, I, \mu}^{\left(I_{1}, \ldots, I_{r}\right)}$ realize $\ell$-adically to the intersection complexes on $\mathrm{Sht}_{N, I, \underline{\mu}}^{\left(I_{1}, \ldots, I_{r}\right)}$ : Roughly, after cutting down the situation to finite-dimensional stacks, the map inv is the map onto the local model, cf. [Laf18, Propositions 2.8, 2.9]. This map is smooth, and thus pullback preserves the intersection complex (up to shift). Next $\pi$ is a small map with connected fibers (cf. [Laf18, Corollary 2.18]), and thus pushforward preserves the intersection complex as well. By construction, the motives $\mathcal{F}_{N, I, \underline{\mu}}^{\left(I_{1}, \ldots, I_{r}\right)}$ depend on a total ordering of $I$, whereas their $\ell$-adic realizations only depend on the ordered partition $I=I_{1} \sqcup \cdots \sqcup I_{r}$, cf. [Laf18, Theorem 1.17, Corollary 2.15, 2.16]. We expect that the same result holds true for the intersection motives, cf. Remark 6.3.7. 


\section{Motives}

In this section we develop motives in the generality we need. In Section 2.1, we list properties of motives on finite-dimensional schemes. This part is mostly expository, except for two aspects: the functors $f_{!}$and $f^{!}$are established for nonseparated maps. This is possible because of a conceptual formulation of $h$-descent for motives. In Section 2.2, we define motives on prestacks. Prestacks are very general 'geometric objects', encompassing (ind-)schemes, and quotients of them by (pro-)algebraic groups, and also (ind-)Artin stacks. In Section 2.3, we prove a comparison result with the !-pushforward for a map of Artin stacks locally of finite type under the $\ell$-adic étale realization. In Section 2.4, we construct a sixfunctor formalism for motives on ind-schemes.

Notation 2.0.1. Throughout Section 2, $S$ is a regular scheme which is of finite type over a scheme $B$ which is Noetherian, excellent, and of dimension at most 2 (for example of $B=\operatorname{Spec}(k)$, a field or the integers). The category of (not necessarily separated) finite type $S$-schemes is denoted $\operatorname{Sch}_{S}$.

2.1. Motives on finite-dimensional schemes. In this subsection, we recall several properties of the category of motives on a scheme $X$ with rational coefficients

$$
\operatorname{DM}(X) \stackrel{\text { def }}{=} \mathrm{DM}(X, \mathbf{Q}) \quad \text { for } X \in \operatorname{Sch}_{S}^{\mathrm{ft}} .
$$

Very briefly, the category $\operatorname{DM}(X)$ is constructed from (unbounded) complexes of étale sheaves of $\mathbf{Q}$-vector spaces on the site $\mathrm{Sm} / X$ of smooth $X$-schemes. In this category, the relation $Y \times{ }_{X} \mathbf{A}_{X}^{1} \cong Y$ is imposed for any $Y \in \mathrm{Sm} / X$. Moreover, tensoring with $\mathbf{P}^{1}$ is made invertible. This category is denoted by $\operatorname{DA}^{\text {ét }}(X, \mathbf{Q})$ in [Ayo14] and by $\mathrm{D}_{\mathbf{A}, \mathrm{e} t}(X, \mathbf{Q})$ in [CD09].

The power of this approach to motives lies in the existence of a sixfunctor formalism. It was first established by Ayoub [Ayo07a, Ayo07b]. Particularly relevant for this paper is the construction of DM due to Cisinski and Déglise [CD09]. These papers phrase many key results in terms of triangulated categories which is not sufficiently structured for the purposes of this paper. Instead, we need to use an $\infty$-categorical formalism, which was worked out by Hoyois [Hoy17] and Khan [Kha16] for the stable homotopy category SH. We now list properties of DM as needed in the paper.

SYNOPSIS 2.1.1. Let $f: X \rightarrow Y$ be a map in $\operatorname{Sch}_{S}^{\mathrm{ft}}$. The category DM of motives with rational coefficients satisfies the following properties.

(i) The category $\operatorname{DM}(X)$ is a stable, presentable, closed symmetric monoidal $\infty$-category with tensor structure denoted $\otimes$ and internal homomorphisms 
denoted Hom. Its monoidal unit is denoted 1 or $1_{X}$. It has all (homotopy) limits and colimits. As usual, the suspension functor is denoted by [1]. (These properties hold since DM is the $\infty$-category associated to the model category denoted $\mathrm{H}_{\mathrm{L}}-$ mod in [CD09, 14.2.7ff].)

(ii) The assignment $X \mapsto \mathrm{DM}(X)$ can be upgraded to a presheaf of symmetric monoidal $\infty$-categories [CD09, Definition 1.1.29]

$$
\mathrm{DM}^{*}:\left(\mathrm{Sch}_{S}^{\mathrm{ft}}\right)^{\mathrm{op}} \rightarrow \mathrm{Cat}_{\infty}^{\otimes}, \quad X \mapsto \mathrm{DM}(X), \quad f \mapsto f^{*}
$$

For each $f$, there is an adjunction

$$
f^{*}: \mathrm{DM}(Y) \rightleftarrows \mathrm{DM}(X): f_{*} .
$$

(iii) If $f$ is smooth, then $f^{*}$ has a left adjoint, denoted $f_{\sharp}$ ([CD09, Definition 1.1.2] with $\mathcal{P}$ consisting of smooth morphisms.)

(iv) The assignment $X \mapsto \operatorname{DM}(X)$ can be upgraded to a presheaf of $\infty$ categories

$$
\mathrm{DM}:\left(\mathrm{Sch}_{S}^{\mathrm{ft}}\right)^{\mathrm{op}} \rightarrow \mathrm{Cat}_{\infty}, \quad X \mapsto \operatorname{DM}(X), \quad f \mapsto f^{!} .
$$

(In [CD09], whenever $f^{!}$or $f_{\text {! }}$ are concerned, $f$ is required to be separated. See Proposition 2.1.14 how to drop this assumption in this point and also the points (vii)-(x), (xii), and (xvi) below.) For each $f$, there is an adjunction

$$
f_{!}: \mathrm{DM}(X) \rightleftarrows \mathrm{DM}(Y): f^{!} .
$$

For any factorization $f=p \circ j$ with $j$ an open immersion and $p$ a proper map, there is a natural equivalence $f_{!} \stackrel{\cong}{\rightarrow} p_{*} j_{\sharp}$. In particular, for $p$ proper, $p !$ is left adjoint to $p_{!}=p_{*}$. (This pair of functors is the hardest to construct. See [Ayo07a, Section 1.6.5] for quasiprojective maps, [CD09, Theorem. 2.4.50, Proposition 2.2.7] in general. These statements are using the language of triangulated categories. See [Hoy17, Section 6.2] or [Kha16, Section 5.2] in the context of $\infty$-categories.)

(v) For the projection $p: \mathbf{G}_{\mathrm{m}, X} \rightarrow X$, and any $M \in \mathrm{DM}(X)$, the map $p_{\sharp} p^{*} M[-1] \rightarrow M[-1]$ in $\operatorname{DM}(X)$ is a split monomorphism. The complementary summand is denoted by $M(1)$. The functor $M \mapsto M(1)$ is an equivalence with inverse denoted by $M \mapsto M(-1)$ [CD09, Definition 2.4.17].

(vi) The category $\operatorname{DM}(X)$ is compactly generated by the objects $t_{\sharp} 1(n), t$ : $T \rightarrow X$ smooth and $n \in \mathbf{Z}$ [Ayo07b, Theorem 4.5.67]. In particular, the monoidal unit $1_{X} \in \operatorname{DM}(X)$ is compact. The functors $f_{\sharp}, f_{*}, f^{*}, f_{!}$, and $f^{!}$preserve compact objects [CD09, Proposition 15.1.4, Theorem 15.2.1]. 
These functors also preserve arbitrary (homotopy) colimits: for $f_{*}$ and $f^{!}$ this follows from compact generation of DM and preservation of compact objects under $f^{*}$ and $f_{!}$, respectively.

(vii) There is a projection formula $\left(f_{!} M\right) \otimes N=f_{!}\left(M \otimes f^{*} N\right)$ [CD09, Theorem 2.4.50].

(viii) If $p: X \rightarrow S$ denotes the structural map, then the dualising functor

$$
\mathrm{D}_{X} \stackrel{\text { def }}{=} \underline{\operatorname{Hom}}\left(-, p^{!} 1\right)
$$

is a (contravariant) involution on the subcategory $\operatorname{DM}(X)^{\mathrm{c}}$ of compact objects, that is, $\mathrm{D}_{X} \circ \mathrm{D}_{X}=$ id. Furthermore, on compact objects, there are equivalences ([CD09, Theorem 15.2.4], this uses all the assumptions in Notation 2.0.1)

$$
\mathrm{D}_{Y} f_{!}=f_{*} \mathrm{D}_{X}, f^{*} \mathrm{D}_{Y}=\mathrm{D}_{X} f^{!} .
$$

(ix) For a closed immersion $i: Z \rightarrow X$ with complement $j: U \rightarrow X$, the (co)units of the adjunctions above assemble into so-called localization homotopy fiber sequences (see around [CD09, Proposition 2.3.3]):

$$
\begin{aligned}
& i_{!} i^{!} \rightarrow \mathrm{id} \rightarrow j_{*} j^{*} \stackrel{[1]}{\rightarrow}, \\
& j_{!} j^{!} \rightarrow \mathrm{id} \rightarrow i_{*} i^{*} \stackrel{[1]}{\rightarrow} .
\end{aligned}
$$

(x) For a cartesian diagram in $\mathrm{Sch}_{S}^{\mathrm{ft}}$

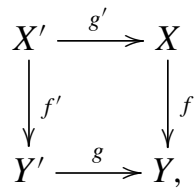

there are natural equivalences (called base change) [CD09, Theorem 2.4.50]:

$$
\begin{aligned}
& g^{!} f_{*} \stackrel{\cong}{\longrightarrow} f_{*}^{\prime} g^{\prime !}, \\
& f^{*} g_{!} \cong g_{!}^{\prime} f^{\prime *} .
\end{aligned}
$$

(xi) The category DM is homotopy-invariant in the sense that for the projection map $p: \mathbf{A}_{X}^{n} \rightarrow X$ for any $n \in \mathbf{Z}_{\geqslant 0}$, the counit and unit maps $p_{\sharp} p^{*} \rightarrow$ id and id $\rightarrow p_{*} p^{*}$ are functorial equivalences in $\operatorname{DM}(X)$ [CD09, 2.1.3].

(xii) If $f$ is smooth of relative dimension $d$, there is a functorial equivalence (called relative purity)

$$
f^{!}=f^{*}(d)[2 d]
$$


(See [Ayo07a, Section 1.6.3] or [CD09, Theorem 2.4.50] or [Hoy17, pages 272-273] for a formulation in the language of $\infty$-categories. The identification of the Thom equivalence as stated follows from the orientability of DM [CD09, Section 14.1.5].)

(xiii) If $X$ is regular,

$$
\operatorname{Hom}_{\operatorname{DM}(X)}\left(1_{X}, 1_{X}(n)[m]\right)=\left(K_{2 n-m}(X) \otimes \mathbf{Q}\right)^{(n)},
$$

where the right term denotes the $n$th Adams eigenspace in the rationalized algebraic $K$-theory of $X$ [CD09, Section 14]. Here $\operatorname{Hom}_{\mathrm{DM}(X)}$ denotes the set of morphisms in the homotopy category. If $X$ is of finite type over a field, this group also identifies with Bloch's higher Chow group $\mathrm{CH}^{n}(X, 2 n-m)_{\mathbf{Q}}$, see [Blo86].

(xiv) The presheaves $\mathrm{DM}^{*}$ and $\mathrm{DM}^{!}$are sheaves for the h-topology (for which see, for example, [Ryd10, Section 8]). That is, if $f: X \rightarrow Y$ is an $h$ covering in $\operatorname{Sch}_{S}^{\mathrm{ft}}$ with Čech nerve $C_{f}^{\bullet} \rightarrow Y, C_{f}^{n}=X^{\times_{Y} n+1}$, the natural map

$$
\operatorname{DM}(Y) \rightarrow \operatorname{DM}^{*}\left(C_{f}^{\bullet}\right):=\lim _{n \in\left(\Delta_{s}\right)^{\text {op }}} \operatorname{DM}\left(C_{f}^{n}\right)
$$

is an equivalence and likewise for $\mathrm{DM}^{!}$. Here lim denotes the limit in the $\infty$-bicategory Cat Cf $_{\infty}$ o-categories. The category $\Delta_{s}$ is the subcategory of the usual simplex category $\Delta$ consisting of injective order-preserving maps. We refer to this property as $h$-descent. (This is discussed in Theorem 2.1.13 below.)

(xv) Suppose $S$ is an excellent scheme and of Krull dimension at most 2 and $X / S$ is separated. The category $\operatorname{DM}(X)$ is equipped with a weight structure $\left(\operatorname{DM}(X)^{\mathrm{w} \leqslant 0}, \operatorname{DM}(X)^{\mathrm{w} \geqslant 0}\right)$. The subcategory $\operatorname{DM}(X)^{\mathrm{w} \leqslant 0}$ is generatedunder extensions, shifts $[n]$ with $n \leqslant 0$, and arbitrary coproducts-by objects of the form $f_{1} 1(n)[2 n]$, where $f: T \rightarrow X$ is proper, $T$ is regular, and $n \in \mathbf{Z}$. Similarly $\operatorname{DM}(X)^{\mathrm{w} \geqslant 0}$ is generated by these objects using shifts $[n]$ for $n \geqslant 0$ instead. If $X$ is regular, $1_{X}$ is in the heart $\operatorname{DM}(X)^{\mathrm{w}=0}=\operatorname{DM}(X)^{\mathrm{w} \leqslant 0} \cap \operatorname{DM}(X)^{\mathrm{w} \geqslant 0}$ of this weight structure. Moreover, $f^{*}$ and $f_{!}$are weight-left exact (preserve ' $\mathrm{w} \leqslant 0$ ') while $f_{*}$ and $f^{!}$are weight-right exact (preserve ' $w \geqslant 0$ '). The weight structure on compact objects is constructed in [Héb11, Theorem 3.8] or [Bon14, Theorem 2.2.1]. See [BS19, Theorem 2.2.1] for how to extend a weight structure on a stable $\infty$-category to its Ind-completion.

(xvi) Let $\ell$ be a prime number invertible on $S$. Then $S$ admits an $\ell$-adic realization functor

$$
\rho_{\ell}: \operatorname{DM}(X) \rightarrow \mathrm{D}_{\text {ét }}\left(X, \mathbf{Q}_{\ell}\right):=\operatorname{Ind}\left(\mathrm{D}_{\text {cons }}^{\mathrm{b}}\left(X, \mathbf{Q}_{\ell}\right)\right)
$$


taking values in the ind-completion of the bounded derived category of constructible $\mathbf{Q}_{\ell}$-adic étale sheaves. This functor is outlined in Section 2.1.2. It commutes with the six functors $f_{*}, f^{*}, f_{!}, f^{!}, \otimes, \underline{\text { Hom. }}$.

REMARK 2.1.10. We emphasize that all work done here is with rational coefficients, cf. Synopsis (xiii). If we were to work with motives with integral coefficients, one can use the category of étale motives which also satisfies the properties listed in Synopsis 2.1.1, except for (xiii) and (xv), cf. [Ayo14, CD16]. Using upcoming work of Spitzweck on $t$-structures on integral Tate motives, the constructions in this paper should carry over and produce a category of mixed motives on the affine Grassmannian $\operatorname{MTM}(\mathrm{Gr}, \mathbf{Z})$ whose rationalization is $\operatorname{MTM}(\mathrm{Gr}, \mathbf{Q})$.

\subsection{1. h-descent and!-functoriality for nonseparated maps.}

LEMMA 2.1.11. Let $F:\left(\mathrm{Sch}_{S}^{\mathrm{ft}}\right)^{\mathrm{op}} \rightarrow \mathrm{Cat}_{\infty}$ be a presheaf such that for any map $f \in \operatorname{Sch}_{S}^{\mathrm{ft}}, f^{+}:=F(f)$ has a left adjoint, denoted $f_{+}$.

Let now $f: X \rightarrow Y$ be a map with Čech nerve $C_{f}^{\bullet}$, that is, $C_{f}^{n}=X^{\times_{Y} n+1}$. If $f$ is such that $f^{+}$is conservative and satisfying the Beck-Chevalley condition, that is, such that for any cartesian diagram

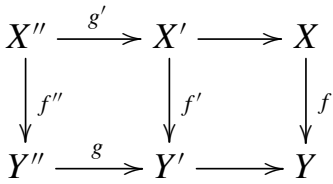

the natural transformation $f_{+}^{\prime \prime} g^{\prime+} \rightarrow g^{+} f_{+}^{\prime}$ is an equivalence, then $F$ satisfies descent with respect to $f$, that is, the following natural functor is an equivalence of $\infty$-categories:

$$
F(Y) \stackrel{\simeq}{\longrightarrow} \lim F\left(C_{f}^{\bullet}\right) .
$$

Proof. This is a slight reformulation of [GR17, Section II.4.7]: the BeckChevalley condition for $F\left(C_{f}^{\bullet}\right)$ is satisfied by assumption, so that Proposition 7.2.2(a) there carries over and shows that $F(Y) \rightarrow \lim F\left(C_{f}^{\bullet}\right)$ is the right adjoint of a monadic adjunction for the functor $\left(p_{2}\right)_{+} p_{1}^{+}: F(X) \rightarrow F(X)$, where $p_{i}: X \times_{Y} X \rightarrow X, i=1,2$ are the projections. Again invoking the BeckChevalley condition, the proof of 7.2.2(b) there carries over and shows that $f_{+}: F(X) \rightleftarrows F(Y): f^{+}$is a monadic adjunction (using that $f^{+}$is continuous and conservative). Both monads are equivalent by invoking the assumption again. 
THEOREM 2.1.13. The presheaves $\mathrm{DM}^{*}$ and $\mathrm{DM}^{!}$are sheaves for the h-topology on $\mathrm{Sch}_{S}^{\mathrm{ft}}$, that is, for an h-covering $f: X \rightarrow Y$, the natural map

$$
\operatorname{DM}(Y) \rightarrow \lim \mathrm{DM}^{!}\left(C_{f}^{\bullet}\right) \quad\left(\text { respectively } \operatorname{DM}(Y) \rightarrow \lim \mathrm{DM}^{*}\left(C_{f}^{\bullet}\right)\right)
$$

are equivalences, where $C_{f}^{\bullet}$ is the $\check{C}$ ech nerve of $f$.

Proof. We apply Lemma 2.1.11. By [CD09, Theorem 14.3.3], $f^{*}$ is conservative for any surjective map. The same proof, dualized, shows the conservativity of $f^{!}$. Since the h-topology is generated by Zariski coverings and proper coverings [Ryd10, Theorem 8.4], it is enough to check the Beck-Chevalley condition in these cases separately.

We begin with $\mathrm{DM}^{!}$: in this case $f_{!}$is left adjoint to $f^{!}$for any separated map $f$ by [CD09, Theorem 2.4.50]. If $f$ is proper, the Beck-Chevalley condition in Lemma 2.1 .11 for $g^{\prime !}$ vs. $f_{!}^{\prime}=f_{*}^{\prime}$ holds true by base change. If $f$ is a disjoint union of open embeddings, $f^{!}=f^{*}$ has the left adjoint $f_{\sharp}$, and again base change holds. For the proper descent for $\mathrm{DM}^{*}$, we apply Lemma 2.1.11 to $\mathrm{DM}^{\text {op }}$, the opposite category. Then the Beck-Chevalley condition is satisfied by proper base change, that is, the equivalence $g^{*} f_{*}^{\prime} \rightarrow f_{*}^{\prime \prime} g^{\prime *}$ for $f$ proper.

In [Ayo07a, Ayo07b, CD09, Hoy17, Kha16], the existence and abovementioned properties of $f_{!}$and $f^{!}$are stated for separated maps $f$ of finite type between schemes. In Theorem 2.3.7, which is applied in Section 6 to the stack of $G$-shtukas, we need the !-pushforward for Deligne-Mumford stacks locally of finite type. The following application of Zariski descent allows to drop this hypothesis, similarly to [LZ11, Example 4.1.10].

PROPOSITION 2.1.14. The adjunction (2.1.3) exists for any (not necessarily separated) map $f: X \rightarrow Y$ in $\mathrm{Sch}_{S}^{\mathrm{ft}}$ in a way such that properties (iv), (vi)(x), (xii) and (xvi) in the above synopsis continue to hold. (Concerning the weight structure in $(x v)$ we do not claim one can drop the separation hypothesis on $X$ since we do not know if de Jong's resolution of singularities can be extended to nonseparated schemes.)

Proof. Given $f$, we can pick open covers $X=\bigcup U_{i}$ and $Y=\bigcup V_{j}$ with $U_{i}$, $V_{j} \in \operatorname{AffSch}_{S}^{\mathrm{ft}}$ so that $f$ restricts to $U_{i} \rightarrow V_{j}$. The latter map is separated, since $U_{i}$ and $V_{j}$ are affine. Let $X_{\bullet}$ and $Y_{\bullet}$ be the Čech nerves of these covers. Each simplicial component $f_{n}$ of the map $f_{\bullet}: X_{\bullet} \rightarrow Y_{\bullet}$ is separated and of finite type.

By Zariski descent (Theorem 2.1.13), $\operatorname{DM}(X)=\lim _{\Delta^{\text {op }}} \operatorname{DM}^{*}\left(X_{\bullet}\right)$. (The * indicates that transition functors in the limit are *-pullbacks along the open immersions.) Using that $\left(f_{n}\right)$ ! and $\left(f_{n}\right)^{!}$commute with $j^{*}$ for an open immersion $j$, $f_{\text {! }}$ (and likewise for $f^{!}$) can be defined to be the unique functors so that the 
following diagram commutes:

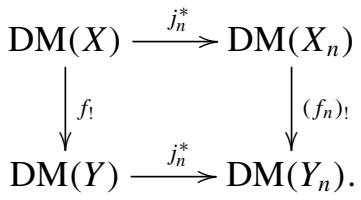

Since (for a Noetherian scheme $X$ ) compactness of an object in $\operatorname{DM}(X)$ is a local condition on $X$, property (vi) extends. Property (viii) extends since $j^{*}$ commutes with Hom [CD09, Theorem 2.4.50(5)] and with $f_{*}$ and $f^{*}$. The latter commutation also saves (xii). Part (ix) carries over as one sees by using in addition that restriction along $\sqcup U_{i} \rightarrow X$ (for an open cover) creates colimits in DM. Likewise (x) continues to hold by taking a covering, as above, of the map $g$ and considering its pullback along $f$. Part (xvi) again carries over by construction of $\mathrm{D}_{\text {et }}\left(X, \mathbf{Q}_{\ell}\right)$ in the nonseparated case in $[\mathbf{L Z 1 1}]$ and the fact that $\rho_{\ell}$ commutes with $j^{*}$.

2.1.2. The $\ell$-adic realization. With $S$ as in Notation 2.0.1, fix a prime number $\ell$ which is invertible in $\mathcal{O}_{S}$. In order to discuss the $\ell$-adic realization we need to use a category of motives with $R$-coefficients, where $R$ is either $\mathbf{Z}$ or $\mathbf{Z} / \ell^{n}$ (as opposed to $R=\mathbf{Q}$ in Synopsis 2.1.1). By the work of Cisinski-Déglise (whose work extends similar results by Ayoub [Ayo14] to more general base schemes), the bulk of the properties in Synopsis 2.1.1 also holds for the category $\mathrm{DM}_{\mathrm{h}}(X, R)$ : (i)-(iii), (v), (xi) hold by construction in [CD16, Section 5.1.1], (iv), (vii), (ix), (x), and (xii) hold by [CD16, Theorem 5.6.2]. As for (vi), the identification of compact objects and constructible objects in $\operatorname{DM}(X, R)$ does hold by [CD16, Theorem 5.6.4] if (and by [CD16, Remark 5.5.11] also only if) the étale cohomological dimensions (for $R$-coefficients) of all residue fields of $X$ are finite. This excludes, say, $X=\operatorname{Spec} \mathbf{Q}$, which is a case we are interested in this paper. However, the following results in [CD16] suggest the slogan that constructible motives should take over the role of compact objects: as for (viii), the dualising functor D is an involution for constructible h-motives by [CD16, Corollary 6.3.15], the remaining formulas in (viii) hold (for arbitrary motives) by [CD16, Theorem 5.6.2]. The identification of Hom-sets with $K$-theory in (xiii) and the weight structure in (xv) do not work for $R=\mathbf{Z}$ or $\mathbf{Z} / \ell^{n}$. As for (xiv), the proof of the h-descent property in Theorem 2.1.13 carries over, the only remaining point being the conservativity of $f^{*}$ for surjective maps $f$. By [CD09, Proposition 2.3.9], we only need to consider finite étale covers and finite surjective radicial maps $f$. For the conservativity in the latter case use [CD16, Proposition 6.3.16]. As is well known, in the former case it holds by construction of $\mathrm{DM}_{h}$ : first embed $\mathrm{DM}_{h}$ in $\underline{D M}_{h}$ [CD16, Definition 5.1.2], 
which is defined as a Bousfield localization (implementing the $\mathbf{P}^{1}$-stabilization) of a category of modules, denoted $R$-mod, in symmetric sequences [CD09, Sections 7.2, 5.3.d]. The left adjoint $f_{\sharp}$ of $f^{*}$ is a left Quillen functor with respect to the stable model structure on the module category $R-$ mod, hence the derived right adjoint of the Bousfield localization commutes with $f^{*}$. The same argument applies for the $\mathbf{A}^{1}$-localization. We hence reduce to modules in symmetric sequences of complexes of $\mathrm{h}$-sheaves. The forgetful functor forgetting the module structure is again conservative and commutes with $f^{*}$, so we end up stating the conservativity for $f^{*}$ (for finite étale maps) on $\operatorname{Shv}_{\mathrm{h}}(\mathrm{Sch} / X)$, which is clear, noting that any h-sheaf is in particular an étale sheaf and vanishing of étale sheaves can be tested stalkwise.

By [CD16, (5.4.1.c), Corollary 5.5.4, Theorem 6.3.11], the étale realization functor for $\ell$-torsion coefficients $\mathrm{DM}_{\mathrm{h}, \text { cons }}(X, \mathbf{Z}) \rightarrow \mathrm{D}_{\text {cons }}^{\mathrm{b}}\left(X, \mathbf{Z} / \ell^{n}\right)$ takes values in the subcategory (always meant to be an $\infty$-category) of complexes of finite Tor-dimension with cohomology sheaves which are constructible in the sense of [SGA73, Exposé IX, Définition 2.3] and vanish in almost all degrees. (At least if $\ell$ is odd and the $\ell$-cohomological dimension of all residue fields of $S$ is finite, it also results from [Ayo14, Theorem 9.7] using the equivalence in [CD16, Corollary 5.5.7, see also Remark 5.5.8].) The category $\mathrm{D}_{\text {cons }}^{\mathrm{b}}\left(X, \mathbf{Z}_{\ell}\right)$ is defined as the full subcategory of $\mathrm{D}\left(X, \mathbf{Z}_{\ell}\right):=\lim \mathrm{D}\left(X, \mathbf{Z} / \ell^{n}\right)$ [LZ14, Sections 0.1 , 1.3] of objects which are of finite Tor-dimension, bounded and constructible in each $\mathbf{Z} / \ell^{n}$-degree [LZ11, Definition 6.3.1]. Taking the limit over $n$, the above realization functor takes values in $\mathrm{D}_{\text {cons }}^{\mathrm{b}}\left(X, \mathbf{Z}_{\ell}\right)$ [Ayo14, Theorem 6.9]. With rational coefficients, the categories $\mathrm{DM}_{\mathrm{h}}^{\mathrm{c}}$ and $\mathrm{DM}^{\mathrm{c}}$ as sketched in the beginning of Section 2.1 are equivalent by [CD16, Theorem 5.2.2, Corollary 5.5.7], so the rationalization of the $\mathbf{Z}_{\ell}$-linear realization gives a functor $\operatorname{DM}(X)^{\mathrm{c}} \rightarrow \mathrm{D}_{\text {cons }}^{\mathrm{b}}(X$, $\mathbf{Q}_{\ell}$ ) [CD16, Remark 7.2.25]. Taking the ind-completion, we arrive at a functor

$$
\rho_{\ell}: \operatorname{DM}(X, \mathbf{Q})=\operatorname{Ind}\left(\operatorname{DM}(X, \mathbf{Q})^{\text {cons }}\right) \rightarrow \operatorname{Ind}\left(\mathrm{D}_{\text {cons }}^{\mathrm{b}}\left(X, \mathbf{Q}_{\ell}\right)\right)=: \operatorname{D}\left(X_{\text {ét }}, \mathbf{Q}_{\ell}\right) .
$$

THEOREM 2.1.15. Suppose S satisfies the assumptions in Notation 2.0.1. Then the presheaf

$$
\mathrm{D}_{\text {ét }}^{!}\left(-, \mathbf{Q}_{\ell}\right):\left(\operatorname{Sch}_{S}^{\mathrm{ft}}\right)^{\mathrm{op}} \rightarrow \mathrm{Cat}_{\infty}, X \mapsto \mathrm{D}_{\text {ét }}\left(X, \mathbf{Q}_{\ell}\right), f \mapsto f^{!}
$$

is a sheaf for the h-topology and likewise for $\mathrm{D}_{\mathrm{cons}}^{\mathrm{b}}\left(-, \mathbf{Q}_{\ell}\right), \mathrm{D}_{\mathrm{cons}}^{\mathrm{b}}\left(-, \mathbf{Z}_{\ell}\right)$ and $\mathrm{D}_{\text {cons }}^{\mathrm{b}}\left(-, \mathbf{Z} / \ell^{n}\right)$.

Proof. Note that $f^{!}$preserves bounded constructible complexes, so the statement makes sense to begin with. By Gabber's work [ILO12, Exposé XIII, Theorem 4.2.3], $f_{*}$ and therefore also $f_{!}$preserves bounded constructible 
complexes for any map $f$ in $\operatorname{Sch}_{S}^{\mathrm{ft}}$. This allows us to adapt the proof of Theorem 2.1.13 to the presheaf given by $\mathrm{D}_{\text {cons }}^{\mathrm{b}}\left(X, \mathbf{Q}_{\ell}\right)$ : for a surjective map $f$, the conservativity of $f^{!}$is reduced to $\mathrm{D}_{\text {cons }}^{\mathrm{b}}\left(X, \mathbf{Z}_{\ell}\right)$ and then to $\mathbf{Z} / \ell^{n}$-coefficients. There, it is reduced to the conservativity of $f^{*}$ using that $f^{!} \mathrm{D}=\mathrm{D} f^{*}$, where $\mathrm{D}$ denotes the dualizing functor, which is an involution on constructible complexes ([ILO12, Exposé XVII, Theorem 0.9], note that the assumptions on the base scheme there are weaker than those in Notation 2.0.1). The conservativity of $f^{*}$ holds since isomorphisms of étale sheaves are detected stalkwise at each geometric point. The Beck-Chevalley condition holds, by proper and smooth base change (again, first for $\mathbf{Z} / \ell^{n}$-coefficients, which formally implies the one for $\mathbf{Z}_{\ell^{-}}$, and then $\mathbf{Q}_{\ell^{-} \text {-coefficients). }}$

This shows the h-descent property for $\mathrm{D}_{\text {cons }}^{\mathrm{b}}\left(X, \mathbf{Q}_{\ell}\right)$. In other words (see the proof of Lemma 2.1.11), for an h-covering map $f: X \rightarrow Y, \mathrm{D}_{\text {cons }}^{\mathrm{b}}\left(Y, \mathbf{Q}_{\ell}\right)=$ $\operatorname{Mod}_{T}\left(\mathrm{D}_{\text {cons }}^{\mathrm{b}}\left(X, \mathbf{Q}_{\ell}\right)\right)$, where $T$ is the monad on the sheaf category on $X$ given by $f^{!} f_{!}$. The functor $f^{!}\left(:=\operatorname{Ind}\left(f^{!}\right)\right): \operatorname{IndD}_{\text {cons }}^{\mathrm{b}}\left(Y, \mathbf{Q}_{\ell}\right) \rightarrow \operatorname{IndD}_{\text {cons }}^{\mathrm{b}}\left(X, \mathbf{Q}_{\ell}\right)$ is conservative by Lemma 2.1.16. The Beck-Chevalley condition for the functors on the ind-completed categories follows formally from the one on $\mathrm{D}_{\text {cons }}^{\mathrm{b}}\left(-, \mathbf{Q}_{\ell}\right)$.

LEMMA 2.1.16. Let $T$ be a monad on an $\infty$-category $\mathcal{D}$. Then the right adjoint $\tilde{U}$ of the adjunction

$$
\tilde{F}: \operatorname{Ind} \mathcal{D} \rightleftarrows \operatorname{IndMod}_{T}(\mathcal{D}): \tilde{U}
$$

(obtained by applying Ind to the free-forgetful adjunction) is conservative.

Proof. It is enough to show that the image of $\tilde{F}$, which is the Ind-extension of the free $T$-module functor $F$, generates $\operatorname{IndMod}_{T}(\mathcal{D})$ under colimits. Indeed, any object in $\operatorname{IndMod}_{T}(\mathcal{D})$ is the (filtered) colimit of objects in $\operatorname{Mod}_{T}(\mathcal{D})$, and any object $d \in \operatorname{Mod}_{T}(\mathcal{D})$ is the colimit of the diagram $\cdots \rightrightarrows(F U)^{2}(d)$ $\rightrightarrows F U(d)$.

2.2. Motives on prestacks. In order to deal with motives on $L^{+} G \backslash L G / L^{+} G$, that is, the double quotient of the loop group by the positive loop group, it is convenient to define motives on a large class of geometric objects, namely prestacks. We follow the method proposed by Gaitsgory and Rozenblyum in the context of quasicoherent and ind-coherent sheaves [GR17, Ch. 3, 0.1.1] and by Raskin for $D$-modules [Ras]. Derived categories of sheaves on more general base 'spaces' have also been used by Scholze [Sch17] in his work on a six-functor formalism for the derived category of étale sheaves on small v-stacks.

2.2.1. Definitions and first properties. Let $\mathrm{AffSch}_{S}^{\mathrm{ft}}$ be the category of schemes $X$ which are affine, and equipped with a map $X \rightarrow S$ of finite type. 
Such schemes $X$ are Noetherian, of finite Krull dimension, and any map $X \rightarrow Y$ in $\operatorname{AffSch}_{S}^{\mathrm{ft}}$ is separated and of finite type. Any (not necessarily affine) scheme $X \rightarrow S$ which is locally of finite type has a Zariski covering by objects in $\mathrm{AffSch}_{S}^{\mathrm{ft}}$. The category $\mathrm{AffSch}_{S}^{\mathrm{ft}}$ is essentially small. Throughout, we replace this category by a small skeleton containing the objects of interest to us. Fixing some regular cardinal $\kappa$, we embed $\mathrm{AffSch}_{S}^{\mathrm{ft}}$ into two larger $\infty$-categories:

$$
\operatorname{AffSch}_{S}^{\mathrm{ft}} \subset \operatorname{AffSch}_{S}^{\kappa} \subset \operatorname{PreStk}_{S}^{\kappa}:=\operatorname{Fun}\left(\left(\mathrm{AffSch}_{S}^{\kappa}\right)^{\mathrm{op}}, \infty-\mathrm{Gpd}\right) .
$$

The (ordinary) category in the middle consists of affine $S$-schemes which are obtained as $\kappa$-filtered limits of objects in $\operatorname{AffSch}_{S}^{\mathrm{ft}}$. Equivalently, $\operatorname{AffSch}_{S}^{\kappa}=$ $\operatorname{Pro}_{\kappa}\left(\mathrm{AffSch}_{S}^{\mathrm{ft}}\right)$ is the $\kappa$-pro-completion. Again, this category is small. Finally, the category of prestacks on the right is the presheaf category in the $\infty$-sense [Lur09, Section 5.1] on this category. It contains the category of affine schemes via the Yoneda embedding, and is generated under colimits by them, cf. [Lur09, Corollary 5.1.5.8].

We now define motives on prestacks adapting [Ras, Definition 2.3]. Following [GR17, Ch. 1, 0.6.11], we write DGCat cont $_{\text {for }}$ fo-category of presentable, stable Q-linear dg- $\infty$-categories with continuous (that is, colimitpreserving) functors.

DEFINITION 2.2.1. The functor

$$
\mathrm{DM}_{\kappa}^{!}:\left(\operatorname{AffSch}_{S}^{\kappa}\right)^{\mathrm{op}} \rightarrow \mathrm{DGCat}_{\mathrm{cont}}
$$

is the left Kan extension of the functor $\mathrm{DM}_{\kappa}^{!}:\left(\mathrm{AffSch}_{S}^{\mathrm{ft}}\right)^{\text {op }} \rightarrow$ DGCat $_{\text {cont }}$ mentioned in Synopsis 2.1.1, (ii). The functor

$$
\mathrm{DM}_{\kappa}^{!}:\left(\operatorname{PreStk}_{S}^{\kappa}\right)^{\mathrm{op}} \rightarrow \text { DGCat }_{\mathrm{cont}}
$$

is the right Kan extension of this functor along the Yoneda embedding.

The Kan extensions exist by [Lur09, Theorem 5.1.5.6] since DGCat cont $_{\text {is }}$ bicomplete [GR17, Section I.1, Corollary 5.3.4], and the category $\operatorname{AffSch}_{S}^{\kappa}$ is small (this is the purpose of the size restriction using $\kappa$ ). Choosing a larger cardinal $\kappa^{\prime}>\kappa$, the restriction of $\mathrm{DM}_{\kappa^{\prime}}^{!}$on $\left(\operatorname{PreStk}_{S}^{\kappa^{\prime}}\right)^{\text {op }}$ to $\left(\operatorname{PreStk}_{S}^{\kappa}\right)^{\text {op }}$ is equivalent to $\mathrm{DM}_{\kappa}$. In this sense, the choice of $\kappa$ does not matter as long $\kappa$ is large enough so that $\operatorname{AffSch}_{S}^{\kappa}$ contains all affine schemes of interest to us. For the purposes of this paper, it is enough to choose $\kappa$ to be the countable cardinal, since the only nonfinite type objects we encounter are countably indexed. We now fix $\kappa$ throughout the document, and drop it from the notation. 
REMARK 2.2.2. (i) For a prestack $X, \operatorname{DM}(X)=\lim _{T \rightarrow X} \operatorname{DM}^{!}(T)$, where the limit is over the category of $S$-maps $T \rightarrow X$ for any $T \in \mathrm{AffSch}_{S}$. Thus, a motive $M$ on $X$ can be thought of system of motives $M_{f}$ for any $T \stackrel{f}{\rightarrow} X$, compatible under !-pullback (and these compatibilities are subject to higher coherence conditions). Next, $\operatorname{DM}(T)=\operatorname{colim}_{T \rightarrow T^{\prime}} \mathrm{DM}^{!}\left(T^{\prime}\right)$, where the colimit is over the category of $S$-maps $T \rightarrow T^{\prime}$ for any $T^{\prime} \in \operatorname{AffSch}_{S}^{\mathrm{ft}}$. The (co)limits are taken in DGCat ${ }_{\text {cont }}$ and are formed using !-pullbacks as transition functors. The inclusion DGCat cont $\subset \mathrm{Cat}_{\infty}$ preserves limits, so that the limit above, and also the one in Theorem 2.1.13 can be taken in DGCat ${ }_{\text {cont }}$

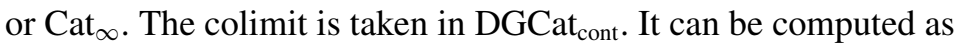

$$
\underset{\text { DGCat }_{\text {cont }}}{\operatorname{colim}} \operatorname{DM}\left(T^{\prime}\right)=\underset{\text { Cat }_{\infty}}{\operatorname{Ind}}\left(\operatorname{colim}_{\cos } \operatorname{DM}\left(T^{\prime}\right)^{\mathrm{c}}\right) .
$$

This is a special case of [GR17, Ch. 1, Corollary 7.2.7], according to which

$$
\underset{\text { DGCat }_{\text {cont }}}{\operatorname{colim}} \operatorname{Ind}\left(\mathcal{C}_{i}\right)=\underset{\operatorname{Cat}_{\infty}}{\operatorname{Ind}}\left(\underset{\mathcal{C}_{i}}{\operatorname{colim}}\right) .
$$

Thus, a compact object $M \in \mathrm{DM}(T)$ can be thought of as being pulled back from some object in $\operatorname{DM}\left(T^{\prime}\right)$ for some $T \rightarrow T^{\prime}$ with $T^{\prime} \in \operatorname{AffSch}_{S}^{\mathrm{ft}}$.

(ii) By construction, there is a functor $f^{!}: \operatorname{DM}(Y) \rightarrow \operatorname{DM}(X)$ for any map of prestacks.

(iii) Let $X=\operatorname{colim}_{i \in I} X_{i}$ be a colimit of prestacks. (Here and elsewhere, unless otherwise mentioned, (co)limits are meant in the $\infty$-categorical sense, also called homotopy (co)limits.) The universal property of the category of $\infty$-presheaves [Lur09, Theorem 5.1.5.6] yields an equivalence

$$
\operatorname{DM}(X)=\lim _{i \in I} \operatorname{DM}^{!}\left(X_{i}\right) .
$$

(iv) It is possible to left/right Kan extend the presheaf $\mathrm{DM}^{*}$ in Synopsis 2.1.1, (ii) to PreStk ${ }_{S}$. However, the computation of motives on an ind-scheme or more generally an ind-Artin stack $X$ in Corollary 2.3.4 only works for DM! We therefore rarely consider the presheaf $\mathrm{DM}^{*}$, and will soon just write $\mathrm{DM}$ instead of $\mathrm{DM}^{!}$. However, there are cases of prestacks $X$ where $\operatorname{DM}^{*}(X)$ and $\mathrm{DM}^{!}(X)$ are equivalent. To give just one example, consider $X=\mathbf{A}_{S}^{\infty}=$ $\lim \left(\cdots \stackrel{p}{\rightarrow} \mathbf{A}_{S}^{2} \stackrel{p}{\rightarrow} \mathbf{A}_{S}^{1} \stackrel{p}{\rightarrow} S\right)$, where the maps are the standard projection maps. Then $\mathbf{A}_{S}^{\infty} \rightarrow S$ is an $S$-affine scheme which is pro-( $S$-smooth). There is an equivalence

$$
\operatorname{DM}^{!}\left(\mathbf{A}_{S}^{\infty}\right)^{\mathrm{c}}=\underset{\mathrm{Cat}_{\infty}}{\operatorname{colim}}\left(\mathrm{DM}(S) \stackrel{p^{!}}{\rightarrow} \operatorname{DM}\left(\mathbf{A}_{S}^{1}\right) \stackrel{p^{!}}{\rightarrow} \cdots\right)
$$


and likewise with ! exchanged by * throughout. Using the natural equivalence $p^{*}(-) \otimes p^{!} 1 \stackrel{\cong}{\Rightarrow} p^{!}(-)$and the $\otimes$-invertibility of $p^{!} 1=1(1)[2]$, we get an equivalence of the above with $\operatorname{DM}^{*}\left(\mathbf{A}^{\infty}\right)^{\mathrm{c}}$, which implies the claim by passing to the ind-completion.

(v) For $X \in \operatorname{Sch}_{S}^{\mathrm{ft}}$, $\mathrm{DM}(X)$ as recalled in Synopsis 2.1.1 agrees with $\mathrm{DM}(X)$ as given by Definition 2.2.1: if $X$ is affine, this is tautological, in general it holds by Zariski descent. Likewise the a priori ambiguity for $\operatorname{DM}(X)$ for $X \in \mathrm{AffSch}_{S}$ Noetherian and of finite Krull dimension (but not necessarily of finite type over $S$ ) is harmless, since $\operatorname{DM}(X)$ as discussed in Synopsis 2.1.1 is equivalent to the $\operatorname{DM}(X)$ obtained by Definition 2.2.1. This is a consequence of the continuity of DM (on finite type $S$-schemes) [CD09, Proposition 14.3.1] and Remark 2.2.2(i).

(vi) Let $X: I \rightarrow$ Sch be a diagram of (Noetherian, finite-dimensional) schemes. Ayoub [Ayo07a] constructs the stable homotopy category $\mathrm{SH}(X)$. Ayoub's approach is based on the category $\mathrm{Sm} / X$ which he defines as the lax colimit of the categories $\mathrm{Sm} / X_{i}(i \in I)$. We did not investigate the precise relation, but one might speculate that once we replace the lax colimit by the colimit and run the usual steps in the construction of SH (taking presheaves, $\mathbf{A}^{1}$ - and Nisnevich localization, $\mathbf{P}^{1}$-stabilization), we would get a category equivalent to $\mathrm{SH}^{*}\left(X_{i}\right):=\operatorname{colim} \mathrm{SH}\left(X_{i}\right)$, where the transition functors are given by the *-pullbacks along the maps in the diagram $X$.

REMARK 2.2.5. Any functor $\left(\mathrm{AffSch}_{S}\right)^{\mathrm{op}} \rightarrow$ Sets is a prestack by regarding it as a simplicially constant presheaf. This includes schemes, ind-schemes (for which see Section 2.4), and also (ind-)algebraic spaces. Likewise, any functor $\left(\mathrm{AffSch}_{S}\right)^{\text {op }} \rightarrow$ Gpd to classical groupoids is a prestack by identifying classical groupoids with 1-truncated spaces. This includes (ind-)Deligne-Mumford or (ind-)Artin stacks. More generally, $n$-geometric stacks [TV08, Section 1.3] (and again, their ind-variants) are prestacks as well.

2.2.2. Equivariant motives. The category PreStk $=\operatorname{PreStk}_{S}$ carries a cartesian monoidal structure. Group objects in PreStk in the sense of [Lur17, Definition 7.2.2.1] are referred to as group prestacks. The monoidal $\infty$-category PreStk is the underlying $\infty$-category of the model category $\mathcal{P} \stackrel{\text { def }}{=} \operatorname{sPSh}\left(\mathrm{AffSch}_{S}\right)$ of simplicial presheaves on $\mathrm{AffSch}_{S}$, equipped with the injective model structure and the pointwise product. This forms a combinatorial, symmetric monoidal model category in which all objects are cofibrant. Thus for a nonsymmetric colored operad $O$ in $\mathcal{P}$, the $\infty$-category of $O$-algebras in the $\infty$-sense is presented by the model category of $O$-algebras in the strict sense [Hau15, Theorem 2.15]. 
In particular, any group object $G \in$ PreStk in the sense of [Lur17, Definition 7.2.2.1] can be strictified and, for given $G$, any action of $G$ on some $X \in \operatorname{PreStk}$ can also be strictified.

The only group prestacks we need in this paper (for example the positive loop group $L^{+} G$ ), are ordinary presheaves of (discrete) groups, which are regarded as simplicially constant prestacks, and therefore group objects in PreStk. However, other interesting examples such as the Picard groupoid operating on the moduli stack of vector bundles on a curve, can also be treated using the notion of group prestacks.

DEFINITION 2.2.6. Suppose a group prestack $G$ acts on the left on a prestack $X$. We write $G \backslash X$ for the homotopy colimit of the $G$-action or, equivalently, the image of $X$ under the left adjoint to the functor PreStk $\rightarrow \operatorname{PreStk}_{G}$ which equips a prestack with a trivial $G$-action. The category of $G$-equivariant motives on $X$ is defined as

$$
\operatorname{DM}(G \backslash X) \text {. }
$$

It is well known (see, for example, [GJ09, Example IV.1.10]) that $G \backslash X$ can be computed as the (homotopy) colimit of the bar construction $\operatorname{Bar}(G, X)$ which is the semisimplicial prestack built out of action maps and projection maps:

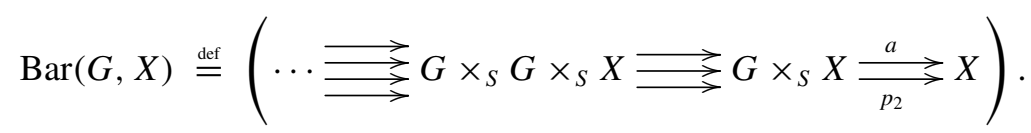

This characterization of $G \backslash X$ and Remark 2.2.2(iii) yield the following result:

LEMMA 2.2.7. If a group prestack $G$ acts on a prestack $X$, there is an equivalence

$$
\operatorname{DM}(G \backslash X) \stackrel{\cong}{\longrightarrow} \lim \operatorname{DM}^{!}(\operatorname{Bar}(G, X)) .
$$

Colloquially speaking, a $G$-equivariant motive is a collection of objects $M_{n} \in$ $\operatorname{DM}\left(G^{n} \times_{S} X\right), n \in \mathbf{Z}_{\geqslant 0}$ together with equivalences $a^{!} M_{0} \stackrel{\simeq}{\longrightarrow} M_{1}, p_{2}^{!} M_{0} \stackrel{\simeq}{\longrightarrow}$ $M_{1}$, and so forth; one for any map in the bar construction, subject to the natural compatibility conditions induced by the relations between the maps.

As was indicated in Remark 2.2.2(iv), it is possible to apply the same considerations to the presheaf $\mathrm{DM}^{*}$ instead of $\mathrm{DM}^{!}$, in which case a $G$-equivariant motive would amount to specifying equivalences $a^{*} M_{0} \cong M_{1}$, and so forth. If $G$ is a smooth algebraic group, the two notions agree, up to equivalence, by the relative purity isomorphism (2.1.8), cf. also the discussion in Remark 3.2.19. 
The next result shows that the existence of adjoint functors on prestack quotients can be checked on the prestacks themselves.

Lemma 2.2.9. Let $G$ be a group prestack, and let $f: X \rightarrow Y$ be a $G$ equivariant map of prestacks. Let $\bar{f}: G \backslash X \rightarrow G \backslash Y$ be the induced map. If $f^{!}: \operatorname{DM}(Y) \rightarrow \operatorname{DM}(X)$ has a left (respectively right adjoint), then the same is true for $\bar{f}^{!}: \operatorname{DM}(G \backslash Y) \rightarrow \operatorname{DM}(G \backslash X)$. This process can be iterated, for example if the left adjoint $f_{!}$of $f^{!}$has another left adjoint, the same holds for the left adjoint $\bar{f}$ ! of $\bar{f}$.

Proof. The categories DM(-) being presentable, we can use the adjoint functor theorem [Lur09, Corollary 5.5.2.9] to construct adjoints. The forgetful functor $\operatorname{DM}(G \backslash X) \rightarrow \operatorname{DM}(X)$ is conservative and preserves (co)limits and therefore also creates them. Thus, the preservation of (co)limits of a functor between categories of $G$-equivariant motives can be checked after forgetting the $G$-equivariance, so that the existence of the adjoints in the nonequivariant setting shows the claim.

Totaro [Tot99, Tot16] and Edidin-Graham [EG98] have introduced equivariant higher Chow groups. We now show that the above equivariant category of motives reproduces these groups (with rational coefficients). To focus on the essential point, suppose $X$ is a smooth finite type scheme of dimension $n$ over a field $k$, equipped with an action of a smooth affine $k$-group scheme $G$ so that the one of the assumptions (1)-(3) in [EG98, Proposition 23] is satisfied. Let $s, t \in \mathbf{Z}$. These assumptions ensure that we may choose a $G$-representation $V$ (viewed as an affine $k$-scheme) and an open subscheme $j: U \subset V$ on which $G$ acts freely such that the reduced complement $i: Z \rightarrow V$ satisfies $c:=\operatorname{codim}_{X} Z>s$ and such that the quotient $(X \times U) / G$ exists in the category of schemes. Let $l:=\operatorname{dim} V / k, g:=\operatorname{dim} G / k$ denote the Krull dimensions. In this case, the definition by Edidin-Graham is in terms of Bloch's higher Chow groups

$$
\mathrm{CH}_{n-s}^{G}(X, t) \stackrel{\text { def }}{=} \mathrm{CH}_{n+l-s-g}((X \times U) / G, t) .
$$

THEOREM 2.2.10. In the above situation, there is an isomorphism

$$
\operatorname{Hom}_{\mathrm{DM}(X / G)}(1,1(s)[2 s-t])=\mathrm{CH}_{\operatorname{dim} X-s}^{G}(X, t) \otimes_{\mathbf{Z}} \mathbf{Q}, \quad s, t \in \mathbf{Z} .
$$

Proof. The freeness assumption ensures that the (homotopy) quotient $(X \times U) / G$ computed in the $\infty$-category of prestacks agrees with the ordinary quotient. Let $p: X \times V \rightarrow V$ be the projection. Using Lemma 2.2.9 (including the notation $\bar{i}:=(i / G)$ for the quotients, and so forth), there is a localization cofiber 
sequence $\bar{i}_{*} \bar{i}^{!} \rightarrow \mathrm{id} \rightarrow \bar{j}_{*} \bar{j}^{\prime}$. By homotopy invariance, $p^{*}$ and therefore $\bar{p}^{*}$ is fully faithful. In order to show that

$$
\begin{aligned}
\operatorname{Hom}_{X / G}(1,1(s)[2 s-t]) & \stackrel{\bar{p}^{*}}{\rightrightarrows} \operatorname{Hom}_{(X \times V) / G}(1,1(s)[2 s-t]) \\
& \stackrel{\bar{j}^{*}}{\rightarrow} \operatorname{Hom}_{(X \times U) / G}(1,1(s)[2 s-t])
\end{aligned}
$$

is an isomorphism, we need to show that the groups $\operatorname{Hom}_{(X \times Z) / G}\left(1, \vec{i}^{!} 1(s)[r]\right)$ vanish for all $r \in \mathbf{Z}$. It is enough to show the same vanishing in $\operatorname{DM}\left(X \times Z \times G^{a}\right)$ for all $a \geqslant 0$. Since the regular locus in $Z$ is nonempty and open [Sta17, Tag 07R5], there exists by Noetherian induction a stratification of $Z$ by regular subschemes (whose codimension in $V$ can only grow), and hence we may assume $Z$ to be regular (use induction and the localization sequence). In this case we have $i^{!} 1=1(-c)[-2 c]$ by absolute purity and the group $\operatorname{Hom}_{\mathrm{DM}\left(X \times Z \times G^{a}\right)}(1,1(s-c)$ $[r-2 c])$ vanishes since $s-c<0$. As $X \times U$ is regular, so is $(X \times U) / G$. We then conclude using $\operatorname{Hom}_{X \times U / G}(1,1(s)[2 s-t])=\mathrm{CH}^{s}(X \times U / G, t) \otimes \mathbf{Q}=$ $\mathrm{CH}_{n+l-g-s}(X \times U / G, t) \otimes \mathbf{Q}$.

For a map of group prestacks $\pi: H \rightarrow G$ and $G$ acting on a prestack $X$ (hence $H$ acts via $\pi)$, there is a restriction functor $\pi^{!}: \operatorname{DM}(G \backslash X) \rightarrow \operatorname{DM}(H \backslash X)$. In the above description, this functor sends a $G$-equivariant motive $\left(M_{n}\right)$ to the !-pullback along $H^{\times{ }_{S}^{n}} \times{ }_{S} X \rightarrow G^{\times s^{n}} \times{ }_{S} X$ in the $n$th component.

The next result serves to cut down the size of $G$ from certain pro-algebraic to algebraic groups. For our conventions on strictly pro-algebraic groups, we refer the reader to Section A.2.

Proposition 2.2.11. Let $G=\lim G_{i}$ be a strictly pro-algebraic $S$-group such that $U:=\operatorname{ker}\left(G \stackrel{\pi}{\rightarrow} G_{0}\right.$ ) is split pro-unipotent (by Definition A.5 a possibly countably infinite successive extension of vector groups). Suppose $G_{0}$ acts on an $S$-scheme $X$. Then the restriction functor $\operatorname{DM}\left(G_{0} \backslash X\right) \rightarrow \operatorname{DM}(G \backslash X)$ is an equivalence.

Proof. We apply Lemma 2.2.12 below to $\pi^{!}: \mathrm{DM}^{!}\left(\operatorname{Bar}\left(G_{0}, X\right)\right) \rightarrow \mathrm{DM}^{!}(\operatorname{Bar}$ $(G, X))$ which in the $m$ th simplicial level is the !-pullback along $\pi^{\times m}: G^{\times m} \times$ $X \rightarrow G_{0}^{\times m} \times X$. Replacing $G_{0}$ by $G_{0}^{m} \times X$ (and $S$ by $X$ ), and so forth, it remains to show that $\pi^{!}: \operatorname{DM}\left(G_{0}\right) \rightarrow \operatorname{DM}(G)$ is fully faithful. By assumption, the map $\pi: G \rightarrow G_{0}$ is a torsor under the split pro-unipotent group $U$. Since the claim is Zariski local on $S$, we may assume that this torsor is trivial by Proposition A.6, so that the map $\pi$ is on the underlying schemes isomorphic to the projection $G_{0} \times$ $U \rightarrow G_{0}$. Replacing $G_{0}$ by $S$, it remains to show that $\pi^{!}: \operatorname{DM}(S) \rightarrow \operatorname{DM}(U)$ is fully faithful. By Definition A.5, we can write $U=\lim U_{i}$ where $\operatorname{ker}\left(U_{i+1} \rightarrow U_{i}\right)$ $=\mathbb{V}\left(\mathcal{E}_{i}\right)$ is a vector group for some $S$-vector bundle $\mathcal{E}_{i}$, see (A.4) for notation. 
By Definition 2.2.1, we have $\operatorname{DM}(U)=\operatorname{colim} \operatorname{DM}\left(U_{i}\right)$, and $\pi^{!}$is the canonical functor into this colimit. Arguing as before, each transition functor is the !-pullback from a vector bundle, and thus fully faithful by homotopy invariance of DM, cf. Synopsis 2.1.1, (xi). Using the equivalence colim DGCat $_{\text {cont }} \operatorname{DM}\left(U_{i}\right)=$ $\operatorname{Ind}\left(\operatorname{colim}_{\mathrm{Cat}_{\infty}} \mathrm{DM}\left(U_{i}\right)^{\mathrm{c}}\right)$ from Remark 2.2.2(i), the full faithfulness of $\pi^{!}$follows from the one of $\operatorname{DM}(S)^{\mathrm{c}} \rightarrow \operatorname{colim}_{\mathrm{Cat}_{\infty}}\left(\operatorname{DM}\left(U_{i}\right)^{\mathrm{c}}\right)$, which in turn follows from the description of the filtered colimit of $\infty$-categories in [Roz] and the full faithfulness of the $\pi_{i}^{!}$.

Lemma 2.2.12 [BN19, Lemma B.6]. Let $C, C^{\prime}: \Delta \rightarrow$ Cat $_{\infty}$ be two cosimplicial $\infty$-categories. Let $F: C \rightarrow C^{\prime}$ be a natural transformation between them, such that each $F_{n}$ is fully faithful and $F_{0}$ is an equivalence. Then $\lim _{\Delta} C \rightarrow \lim _{\Delta} C^{\prime}$ is an equivalence.

EXAMPLE 2.2.13. (i) For a split unipotent $S$-group scheme $U$ (that is, a smooth affine $S$-group scheme which is a successive extension of vector groups) acting trivially on $X$, Proposition 2.2.11 shows that $\operatorname{DM}_{U}(X)=\operatorname{DM}(X)$.

(ii) Proposition 2.2.11 applies to any parahoric subgroup $\mathcal{P}=\lim _{i} \mathcal{P}_{i}$ of $L G$ by Lemma 4.2.7 below. More generally, it applies to any pro-algebraic group which is constructed as a positive loop group along some Cartier divisor, cf. Proposition A.9 and Example A.12 below.

2.2.3. Descent for motives. We now study consequences of descent. Let $\tau$ be a Grothendieck topology on $\mathrm{AffSch}_{S}$ such that each covering family has a refinement by a finite covering family. We now fix $S$, and write $\operatorname{Stk}^{\tau}=\mathrm{Stk}_{S}^{\tau}$ for the category of $\tau$-stacks. By definition [Lur09, Definition 6.2.2.6], this is the full subcategory of PreStk of objects $X$ : $\left(\mathrm{AffSch}_{S}\right)^{\text {op }} \rightarrow \infty$-Gpd which commute with finite coproducts, and such the natural map

$$
X(T) \rightarrow \lim \left(X(U) \Longrightarrow X\left(U \times_{T} U\right) \stackrel{\rightrightarrows}{\rightrightarrows}\right)
$$

is an equivalence for all $\tau$-covers $U \rightarrow T$ in $\operatorname{AffSch}_{S}$. We denote the sheafification (or localization) functor PreStk $\rightarrow \operatorname{Stk}^{\tau}$ by $X \mapsto X^{\tau}$ which is left adjoint to the inclusion $\mathrm{Stk}^{\tau} \subset$ PreStk, cf. [Lur09, Proposition 6.2.2.7].

We are interested in topologies $\tau$ contained in the $h$-topology for which we recall the definition from [Ryd10, Definition 8.1].

Definition 2.2.15. The $h$-topology on $\mathrm{AffSch}_{S}$ (respectively on $\mathrm{Sch}_{S}$ ) is the minimal Grothendieck topology generated by the following covering families 
- Families of open immersions $\left\{U_{i} \stackrel{f_{i}}{\rightarrow} T\right\}$ such that $T=\cup f_{i}\left(U_{i}\right)$.

- Finite families $\left\{U_{i} \stackrel{f_{i}}{\rightarrow} T\right\}$ such that $\sqcup f_{i}: \sqcup U_{i} \rightarrow T$ is universally subtrusive (that is, a $v$-cover) and of finite presentation.

By [Ryd10, Theorem 8.4], any $h$-covering $\left\{U_{i} \rightarrow T\right\}$ of a quasicompact scheme can be refined by a finite covering family $\left\{V_{i} \rightarrow T\right\}$. Thus, as each object in $\mathrm{AffSch}_{S}$ is quasicompact, the $h$-topology on $\mathrm{AffSch}_{S}$ is generated by finite covering families.

THEOREM 2.2.16. Suppose $\tau$ is a Grothendieck topology which is contained in the h-topology, and such that each covering family admits a refinement by a finite covering family (for example, the fppf, étale or Zariski topology, but also the qfh, cdh or h-topology). For any prestack $X \in$ PreStk $_{S}$, !-pullback along the natural map $X \rightarrow X^{\tau}$ yields an equivalence

$$
\mathrm{DM}\left(X^{\tau}\right) \stackrel{\cong}{\longrightarrow} \mathrm{DM}(X) .
$$

Proof. Since the map $X \rightarrow X^{\tau}$ is an equivalence after $h$-sheafification, we may assume that $\tau$ equals the $h$-topology. Now let $T \in \mathrm{AffSch}_{S}$, and suppose that $u: U \rightarrow T$ is an $h$-cover of schemes with $U$ being quasicompact. We claim that the natural map

$$
\operatorname{DM}(T) \rightarrow \lim \operatorname{DM}\left(C_{u}^{*}\right)
$$

is an equivalence, where $C_{u}^{\bullet}$ is the Čech nerve of $u$. If $T$ (and hence $U$ ) is of finite type over $S$, this is a special case of Theorem 2.1.13. Now the proof in [Ras, Proposition 3.12.1] carries over. We briefly recall Raskin's argument: as $U \rightarrow T$ is an $h$-cover of qcqs schemes, one may treat the case of proper surjective morphisms, and finitely presented Zariski coverings separately, cf. [Ryd10, Theorem 8.4]. One shows that $u^{!}$has a left adjoint $u_{\text {! }}$ (for $u$ proper), respectively a right adjoint denoted $u_{*}$ (for $u$ being a quasicompact Zariski covering map). The existence of these adjoints follows from proper, respectively smooth base change

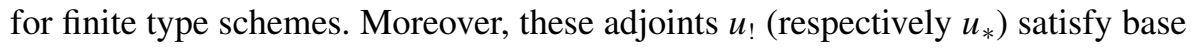
change with respect to $f^{!}$for arbitrary maps of qcqs schemes. Then, the BeckChevalley type argument already used in the proof of Theorem 2.1.13 finishes the proof of (2.2.18).

The universal property of the sheaf category $\mathrm{Stk}_{S}^{\tau}$ [Lur09, Proposition 5.5.4.15, Definition 6.2.2.6] states that it is the localization of PreStk ${ }_{S}$ with respect to maps colim $C_{u}^{\bullet} \rightarrow T$ from the Čech nerve of $h$-coverings $u$ as above, and with respect to finite coproducts $\amalg j\left(T_{i}\right) \rightarrow j\left(\amalg T_{i}\right)$, where $j: \mathrm{AffSch}_{S} \rightarrow \mathrm{PreStk}_{S}$ is the Yoneda embedding and $T_{i} \in \mathrm{AffSch}_{S}$. By (2.2.18), the functor DM! $\mathrm{PreStk}_{S}^{\mathrm{op}} \rightarrow$ DGCat $_{\text {cont }}$ sends the map $\operatorname{colim} C_{u}^{\bullet} \rightarrow T$ to equivalences. Moreover, DM sends 
(finite) coproducts of affine schemes to products, so that DM factors over the sheafification $X \mapsto X^{\tau}$.

EXAMPLE 2.2.19. Let $f: X \rightarrow Y$ be a schematic $h$-covering of prestacks, that is, for any affine scheme $T \rightarrow Y, X \times_{Y} T$ is a scheme, which is an $h$-cover of $T$ in the usual sense. Let $C_{f}^{\bullet}$ be the Čech nerve of $f$. Then there is an equivalence

$$
\operatorname{DM}^{!}(Y) \stackrel{\cong}{\longrightarrow} \lim \operatorname{DM}^{!}\left(C_{f}^{\bullet}\right) .
$$

Indeed, this follows from Remark 2.2.2(iii) and Theorem 2.2.16 using that colim $C_{f}^{\bullet} \rightarrow Y$ is an equivalence after $\tau$-sheafification.

For later use we record the following lemma.

LEMMA 2.2.21. Let $\tau$ be a Grothendieck topology as in Theorem 2.2.16. Let $H \subset$ $G$ be an inclusion of group $\tau$-stacks, and consider the quotient $X:=(G / H)^{\tau}$. Let $e: S / H \rightarrow G \backslash X$ be the map in PreStk induced by the base point $S \rightarrow X$. Then there is an equivalence

$$
e^{!}: \operatorname{DM}(G \backslash X) \rightarrow \operatorname{DM}(S / H) .
$$

Proof. The map $G / H \rightarrow X$ is an equivalence after $\tau$-stackification, hence so is $e: S / H=G \backslash G / H \rightarrow G \backslash X$. The lemma follows from Theorem 2.2.16.

In the definition of $G \backslash X$, there is no consideration of a topology on $\operatorname{AffSch}_{S}$. Consequently, $G \backslash X$ is only a prestack. Now suppose $G \in \mathrm{Stk}^{\tau}$ for some Grothendieck topology $\tau$. The category of $\tau$-stacks with $G$-action $\operatorname{Stk}_{G}^{\tau}$ is the full subcategory of PreStk ${ }_{G}$ whose objects are $\tau$-stacks. The following general statement about principal bundles in an $\infty$-topos is well known, see for example [NSS15, Proposition 3.7] for the implication (i) $\Rightarrow$ (ii).

LEMmA 2.2.22. Let $X \in \mathrm{Stk}^{\tau}$ equipped with the trivial $G$-action. The following data are equivalent:

(i) An object $P \in \mathrm{Stk}_{G}^{\tau}$ together with an equivalence $\alpha$ : $(G \backslash P)^{\tau} \simeq X$.

(ii) An effective epimorphism $p: P \rightarrow X$ in $\operatorname{Stk}_{G}^{\tau}$ such that $G \times P \simeq P \times_{X} P$, $(g, p) \mapsto(g p, p)$ is an equivalence.

In this case, the object $P \rightarrow X$ is called a G-torsor in the $\tau$-topology.

Proof. Let $P \rightarrow X$ be any map in an $(\infty, 1)$-topos with Čech nerve $P^{\bullet}$. By [Lur09, Corollary 6.2.3.5], $P \rightarrow X$ is an effective epimorphism if and only if the canonical map colim $P^{\bullet} \rightarrow X$ is an equivalence. Now consider a pair 
as in (i). Being a left adjoint, $\tau$-sheafification commutes with colimits so that $\pi: P \rightarrow(G \backslash P)^{\tau}$ is an effective epimorphism (the Čech nerve of $P \rightarrow G \backslash P$ is the bar construction, and $G \backslash P$ its colimit). Hence, passing to the Čech nerve of $\pi$ the equivalence $\alpha$ induces on the first simplicial level the equivalence $G \times P \simeq P \times_{X} P$. Conversely, having $P \rightarrow X$ as in (ii), we use that both the bar construction and the Čech nerve are 1-coskeletal and conclude that all simplicial levels in the Čech nerve are equivalent to the bar construction. Taking colimits, we find a pair as in (i).

A morphism between $G$-torsors $P \rightarrow P^{\prime}$ over $X$ is a morphism in the slice category $\mathrm{Stk}_{G}^{\tau} / X$. A $G$-torsor equivalent to $G \times X$ is called trivial. Clearly, this happens if and only if $P \rightarrow X$ admits a section (use the equivalence in Lemma 2.2.22(ii)).

Lemma 2.2.23. Let $T$ be an affine scheme. Then any $G$-torsor $P \rightarrow T$ in the $\tau$-topology is $\tau$-locally on $T$ trivial.

Proof. View $T \simeq(G \backslash P)^{\tau}$ as an element in $s_{T} \in(G \backslash P)^{\tau}(T)$. By the description of sheafification in [Lur09, Section 6.5.3, page 673 and proof of Proposition 6.2.2.7], there exists a $\tau$-cover $T^{\prime} \rightarrow T$, and a lift $s_{T^{\prime}}$ to $G\left(T^{\prime}\right) \backslash P\left(T^{\prime}\right)$, and thus $P\left(T^{\prime}\right) \neq \varnothing$. This shows that the base change $P_{T^{\prime}} \rightarrow T^{\prime}$ is trivial.

COROLlary 2.2.24. Let $X$ be any $\tau$-stack. Then every morphism of $G$-torsors $P \rightarrow P^{\prime}$ over $X$ is an equivalence.

Proof. This can be tested after pullback $T \rightarrow X$ for $T \rightarrow$ AffSch $_{S}$. By descent, we may work $\tau$-locally on $T$. By Lemma 2.2.23, we may assume that both torsors $P, P^{\prime}$ are trivial. Then the corollary is clear.

Similarly to [Sta17, Tag 04WL], we consider for each $T \in \mathrm{AffSch}_{S}$ the $\infty$ category $[G \backslash X]^{\tau}(T)$ defined as the full subcategory of the slice $\mathrm{Stk}_{G}^{\tau} / X \times T$ of those objects $b \times a: P \rightarrow X \times T$ where $a$ is a $G$-torsor in the $\tau$-topology. By [Lur09, Corollary 2.4.7.12], the formation $T \mapsto \mathrm{Stk}_{G}^{\tau} / X \times T \in \mathrm{Cat}_{\infty}$ is functorial in the $\infty$-sense, so that $[G \backslash X]^{\tau}$ is a presheaf of $\infty$-categories, and by Corollary 2.2.24 it takes values in $\infty$-groupoids. Also being a $\tau$-sheaf, we see that $[G \backslash X]^{\tau}$ defines $\tau$-stack.

Proposition 2.2.25. Let $X \in \mathrm{Stk}_{G}^{\tau}$. In the above situation, the natural map given by the trivial $G$-torsor on $X$,

$$
X \rightarrow[G \backslash X]^{\tau},
$$


is a $G$-torsor in the $\tau$-topology, so that (by Lemma 2.2.22) $(G \backslash X)^{\tau}=[G \backslash X]^{\tau}$. In particular, if $\tau$ is contained in the h-topology (Theorem 2.2.16), there is an equivalence

$$
\mathrm{DM}\left([G \backslash X]^{\tau}\right) \stackrel{\cong}{\longrightarrow} \mathrm{DM}(G \backslash X) .
$$

Proof. We check Lemma 2.2.22(ii). Clearly, $G \times X \simeq X \times_{[G \backslash X]^{\tau}} X$, and it remains to show that $X \rightarrow[G \backslash X]^{\tau}$ is an effective epimorphism. By [Lur09, Proposition 7.2.1.14], this can be tested on the 0-truncation, that is, on its underlying 1-topos. But a map of ordinary $\tau$-sheaves is an effective epimorphism if it is an epimorphism. Thus, Lemma 2.2.23 implies the proposition.

REMARK 2.2.26. We apply Proposition 2.2.25 to quotients like $L^{+} G \backslash L G / L^{+} G$, that is, the double quotient of the loop group by the positive loop group. In virtue of Section A.4 below each $L^{+} G$-torsor for the fpqc topology admits sections étale-locally so that it is enough to consider étale sheafifications, cf. Lemma 5.3.2 below.

2.3. Motives on ind-Artin stacks. In Section 6 below, we construct intersection motives on moduli stacks of shtukas. The following framework is convenient for our constructions.

Let IndArt ${ }_{S}^{\text {lft }}$ be the category of strict ind-Artin stacks ind-(locally of finite type) over $S$. By definition, every object $X \in \operatorname{IndArt}_{S}$ admits a presentation over a countable filtered index set

$$
X=\underset{i \in I}{\operatorname{colim}} X_{i}
$$

by $S$-Artin stacks locally of finite type as defined in [Sta17, Tag 0260] with transition maps $t_{i, j}: X_{i} \rightarrow X_{j}$ being closed immersions for all $i \leqslant j$. The category $\operatorname{IndArt}_{S}^{\mathrm{lft}}$ is, by definition, a full subcategory of the $(2,1)$-category of presheaves of ordinary groupoids on $\mathrm{AffSch}_{S}$. As was mentioned in Remark 2.2.5, we regard it as a full subcategory of PreStk ${ }_{S}$. In (2.3.1), colim denotes the colimit of presheaves of ordinary groupoids. The inclusion $\tau_{\leqslant 1} \operatorname{PreStk}_{S} \subset \operatorname{PreStk}_{S}$ preserves filtered colimits [Lur09, Corollary 5.5.7.4], so we will not distinguish between them. Any object (2.3.1) is automatically a sheaf of groupoids in the fppf topology on $\mathrm{AffSch}_{S}$ because each $X_{i}$ is by definition, and the colimit by [EG15, Lemma 4.2.6] (each object in $\mathrm{AffSch}_{S}$ is quasicompact). (Even in the fpqc topology if the diagonal of each $X_{i}$ is quasiaffine.)

LEMMA 2.3.2 (Lurie [Gai, Lemmas 1.3.3, 1.3.6]). Let I be an $\infty$-category and $F: I \rightarrow$ DGCat $_{\text {cont }}$ a functor. For a map $\alpha: i \rightarrow j$ in $I$, the right adjoint of $F(\alpha)$ (which exists by the adjoint functor theorem) is denoted $G(\alpha)$. 
(i) The evaluation functors $\lim _{I^{\mathrm{op}}} G \stackrel{\mathrm{ev}_{i}}{\longrightarrow} G(i)$ admit left adjoints. These left adjoints assemble to an equivalence of $\infty$-categories

$$
\underset{I}{\operatorname{colim}} F \stackrel{\cong}{\longrightarrow} \lim _{I^{\mathrm{op}}} G \text {. }
$$

Here and below, the colimit is taken in the $\infty$-category DGCat cont $_{\text {of }}$ presentable DG-categories with continuous (that is, colimit-preserving) functors.

(ii) If $I$ is filtered and the $G(\alpha)$ are also continuous, then the following composition of the natural functors and this equivalence, $F(j) \stackrel{\text { ins }_{j}}{\longrightarrow}$ $\operatorname{colim}_{I} F \cong \lim _{I \text { op }} G \stackrel{\mathrm{ev}_{i}}{\longrightarrow} G(i)$, can be computed as

$$
\operatorname{colim}_{k \in I, \alpha: j \rightarrow k, \beta: i \rightarrow k} G(\beta) \circ F(\alpha) .
$$

Proposition 2.3.3. Let $f: X \rightarrow Y$ in IndArt $_{S}^{\text {lft }}$ be any map.

(i) The functor $f^{!}: \mathrm{DM}(Y) \rightarrow \operatorname{DM}(X)$ has a left adjoint $f_{!}$.

(ii) If $f$ is ind-proper, then $f_{!}$satisfies base change with respect to $g$ ! for any map $g: Y^{\prime} \rightarrow Y$ of prestacks.

(iii) If $f$ is representable by a closed immersion, $f_{!}$is fully faithful.

Proof. (i): All $\infty$-categories in sight are presentable, so we can apply the adjoint functor theorem [Lur09, Corollary 5.5.2.9] once we know that $f^{!}$preserves all limits and filtered colimits.

If $f$ is a map between $S$-schemes locally of finite type, we pick a (possibly infinite) Zariski cover $V=\bigsqcup V_{i} \stackrel{v}{\rightarrow} X$ by open subschemes $V_{i} \in \operatorname{AffSch}_{S}^{\mathrm{ft}}$, and similarly $u: U \rightarrow Y$ together with a map $g: V \rightarrow U$ compatible with $f$. Theorem 2.2.16 gives an equivalence $\operatorname{DM}(Y)=\lim \operatorname{DM}\left(C_{u}^{\bullet}\right)$ and likewise for $X$. Applying Lemma 2.3.2(i) to the composite

$$
\Delta \stackrel{C_{v}^{*}}{\longrightarrow} \operatorname{Sch}_{S}^{\text {op }} \stackrel{\text { DM! }^{!}}{\longrightarrow} \text { DGCat }_{\text {cont }}
$$

we see that the forgetful functor $\lim _{n} \operatorname{DM}\left(C_{v}^{n}\right) \rightarrow \mathrm{DM}(V)$ has a left adjoint, so it preserves limits. Being a functor in DGCat ${ }_{\text {cont }}$, it also preserves colimits. Being conservative, it therefore creates (co)limits. To check preservation of (co)limits

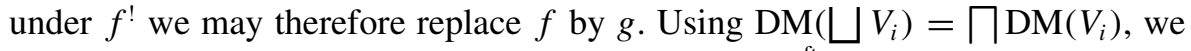
further reduce to the case that $X$ and $Y$ are in $\mathrm{AffSch}_{S}^{\mathrm{ft}}$, in which case we know the desired properties of $f^{!}$by Synopsis 2.1.1, (iv) and (vi). 
If $f$ is a map between algebraic spaces locally of finite type over $S$, we choose an étale cover $u: U \rightarrow Y$ by a scheme $U$ and an étale cover $v: V \rightarrow X$ and a map $g: V \rightarrow U$ so that $u \circ g=f \circ v$. Using the étale descent equivalences $\operatorname{DM}(X)=\lim \operatorname{DM}\left(C_{v}^{\bullet}\right)$ and likewise for $Y$, we repeat the above argument and reduce the claim to the previously considered case.

This reasoning can be repeated with Artin stacks instead of algebraic spaces. Here we use that any smooth cover of an Artin stack $u: U \rightarrow X$ by an algebraic space defines an effective epimorphism of étale stacks (by [Lur09, Proposition 7.2.1.14] this can be checked on the 0th truncation), so that the natural map colim $C_{u}^{\bullet} \rightarrow X$ is an equivalence after étale sheafification. Then by Theorem 2.2.16 and Remark 2.2.2, we obtain $\operatorname{DM}(X)=\operatorname{DM}\left(\left(\operatorname{colim} C_{u}^{\bullet}\right)^{\text {ét }}\right)=$ $\operatorname{DM}\left(\operatorname{colim} C_{u}^{\bullet}\right)=\lim \operatorname{DM}\left(C_{u}^{\bullet}\right)$, and we repeat the reasoning as before.

Finally, suppose both $X$ and $Y$ are ind-Artin stacks. Choosing suitable presentations of $X$ and $Y$, the map $f$ is a colimit of maps $f_{i}: X_{i} \rightarrow Y_{i}, i \in I$ over the same index set, cf. Appendix A.1. Again, the evaluation functors $\mathrm{ev}_{i}^{X}: \mathrm{DM}(X) \rightarrow \mathrm{DM}\left(X_{i}\right)$ and likewise for $Y_{i}$ preserve (co)limits. They satisfy $\operatorname{ev}_{i}^{X} f^{!}=f_{i}^{!} \mathrm{ev}_{i}^{Y}$. The family of the $\mathrm{ev}_{i}^{?}$ for all $i$ is conservative, so that again preservation of (co)limits under $f^{!}$can be checked for $f_{i}^{!}$instead.

The full faithfulness for a closed immersion $f$, that is, $f^{!} f_{!}=$id, is again checked on each $X_{i}$ in an ind-presentation (2.3.1), and then on smooth, respectively étale atlases. We then conclude using the corresponding property for motives on finite type $S$-schemes (combine Synopsis 2.1.1(ix) and (x)).

(ii): If $f$ is a proper map of locally finite type $S$-schemes, then the left adjoint $f !$ in the first step is given by

$$
\lim _{n}\left(\mathrm{DM}\left(C_{u}^{n}\right) \stackrel{g_{!}^{n}}{\longrightarrow} \mathrm{DM}\left(C_{v}^{n}\right)\right),
$$

where $g^{n}$ is the $n$th simplicial level of the map between the two Čech nerves. Indeed, this functor is well-defined by the proper base change (for finite type $S$-schemes) and it is left adjoint to $f^{!}$since this is true in each level of the Čech nerve. Applying this argument to each step in the above construction, we obtain our claim for $f$ being an ind-proper map in $\operatorname{IndArt}_{S}^{\mathrm{lft}}$.

This way, the claim $f^{!} f_{!}=$id for a closed immersion $f$ in $\operatorname{IndArt}_{S}^{\text {lft }}$ also reduces to the same claim for $f$ in $\operatorname{AffSch}_{S}^{\mathrm{ft}}$, where we know it from Synopsis 2.1.1(ix) and $(\mathrm{x})$.

The preceding statements allow to rephrase motives on ind-Artin stacks in a way which is more closely reminiscent of the usual definition of derived categories on ind-Artin stacks. 
COROLlary 2.3.4. For any presentation of an ind-Artin stack $X$ as in (2.3.1), the category of motives on $X$ can be computed in two ways:

$$
\underset{t !}{\operatorname{colim}} \operatorname{DM}\left(X_{i}\right)=\lim _{t^{!}} \operatorname{DM}\left(X_{i}\right)=\operatorname{DM}(X) .
$$

If an S-group prestack $G$ acts on $X$ and the presentation is $G$-equivariant, then there is an equivalence

$$
\underset{t !}{\operatorname{colim}} \operatorname{DM}\left(G \backslash X_{i}\right) \stackrel{\cong}{\longrightarrow} \lim _{t^{!}} \operatorname{DM}\left(G \backslash X_{i}\right) \stackrel{\cong}{\longrightarrow} \operatorname{DM}(G \backslash X) .
$$

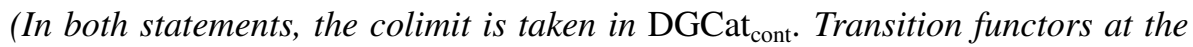
left are the $\left(t_{i j}\right)_{!}$. In the middle, the transition functors are the $t_{i j}^{!}$.)

Proof. The formulations using the limits follow from Remark 2.2.2(iii) because $G \backslash X=\operatorname{colim}\left(G \backslash X_{i}\right)$. Then, use Lemma 2.3.2.

By Corollary 2.3.4, motives $M \in \mathrm{DM}(X)$ can informally be thought of as sequences $M_{i} \in \mathrm{DM}\left(X_{i}\right)$, together with equivalences $M_{i} \rightarrow t_{i, j}^{!} M_{j}$ for $i \leqslant j$. These equivalences are subject to higher coherence conditions. Combining Lemma 2.3.2(ii) and the full faithfulness of the $\left(t_{i, j}\right)_{!}$, a motive of the form ins ${ }_{i}(N)$ is given by $t_{i, j}^{!} N$ in degrees $j \leqslant i$ and $\left(t_{i, j}\right)_{!} N$ for $j \geqslant i$. We say that $M$ is supported on $X_{i}$ if it is of the form $M=\operatorname{ins}_{i}(N)$ for some $N \in \operatorname{DM}\left(X_{i}\right)$.

LEMMA 2.3.6. For any ind-Artin stack $X$, the category $\mathrm{DM}(X)$ is compactly generated. An object $M \in \mathrm{DM}(X)$ is compact if and only if it is of the form $M=\operatorname{ins}_{i}(N)$ for some $i$ and some compact object $N \in \operatorname{DM}\left(X_{i}\right)^{\mathrm{c}}$. (Thus, it is supported on $X_{i}$, and is a compact object there.)

Proof. We retrace the proof of Proposition 2.3.3: if $X$ is an algebraic space with atlas $v: V \rightarrow X, \operatorname{DM}(X)=$ colim $\mathrm{DM}_{!}\left(C_{v}^{\bullet}\right)$ is compactly generated by (2.2.4). Here we use that the !-pushforwards and !-pullbacks along the maps in the Čech nerve $C_{v}^{\bullet}$ preserve compact objects. From there, we obtain the claim for Artin stacks $X$ in the same vein. Similarly, for an ind-Artin stack, use Corollary 2.3.4.

Since the pushforwards $\left(t_{i, j}\right)$ ! are fully faithful, the mapping space $\operatorname{Hom}_{\mathrm{DM}(X)}$ $(M, N)$ between two compact objects is given by $\operatorname{Hom}_{\mathrm{DM}\left(X_{i}\right)}\left(M_{i}, N_{i}\right)$ for some $i \gg 0$. Summarizing this discussion, we can say that a compact object in $\operatorname{DM}(X)$ is nothing but a motive $M \in \operatorname{DM}\left(X_{i}\right)^{\mathrm{c}}$ for some $i \in I$ (and it is identified with its image under $\left(t_{i, j}\right)$ ! for $j \geqslant i$ ).

The following theorem compares the motivic functor $f_{\text {! }}$ with its counterpart for étale $\ell$-adic sheaves due to Liu and Zheng. Liu-Zheng's work is a $\infty$-categorical 
refinement of constructions by Laszlo and Olsson [LO08]. The formalism for $\mathbf{Z}_{\ell}$-adic étale sheaves in [LZ14, Section 2.3] extends to the case of $\mathrm{D}_{\text {ét }}\left(X, \mathbf{Q}_{\ell}\right)$ in view of Theorem 2.1.15 and the fact that for smooth maps (which are the ones needed to cover Artin stacks by schemes) $f^{*}$ and $f^{!}$are equivalent up to twist and shift.

We only state the comparison for Artin stacks as opposed to ind-Artin stacks, since the authors in [LZ14] do not consider ind-objects. We apply the theorem to identify the $\ell$-adic realization of the intersection cohomology motives of moduli stacks of shtukas with the $\ell$-adic intersection cohomology complex as for example considered in [Laf18, Définition 4.1].

THEOREM 2.3.7. Let $f: X \rightarrow Y$ be a map in $\mathrm{Art}_{S}^{\mathrm{ft}}$, and let $\ell$ be a prime invertible on $S$.

(i) The $\ell$-adic realization functor $\rho:=\rho_{\ell}$ for motives on $S$-schemes of finite type (see Section 2.1.2) extends to an $\ell$-adic realization functor

$$
\rho_{X}: \operatorname{DM}(X) \rightarrow \mathrm{D}_{\text {ét }}\left(X, \mathbf{Q}_{\ell}\right):=\operatorname{Ind}\left(\mathrm{D}_{\text {cons }}^{\mathrm{b}}\left(X, \mathbf{Q}_{\ell}\right)\right)
$$

taking values in the ind-completion of the derived $\infty$-category of $\ell$-adic constructible sheaves constructed in [LZ14, Section 2.3].

(ii) The square

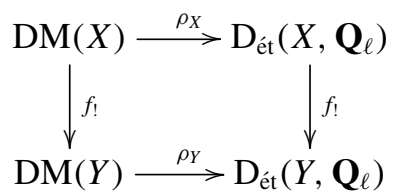

commutes up to equivalence, that is, there is an equivalence $f_{!} \circ \rho_{X} \stackrel{\cong}{\rightarrow}$ $\rho_{Y} \circ f_{!}$.

Proof. We instead show these claims when $\mathrm{D}_{\text {ét }}\left(-, \mathbf{Q}_{\ell}\right)$ is replaced by $\mathrm{D}(-, \mathbf{Z} / m)$, $m:=\ell^{n}$ and when DM is replaced by its integral analogue, denoted $\mathrm{DM}_{\mathrm{h}}$ in [CD16, Definition 5.1.3] (alternatively, in view of [CD16, Corollary 5.5.7], one may use Ayoub's category DAét of étale motives without transfers [Ayo14, Section 3] if $\ell$ is odd and the $\ell$-cohomological dimension of all residue fields in $S$ is finite). The rather formal extension to $\mathbf{Z}_{\ell^{-}}$and then $\mathbf{Q}_{\ell}$-adic coefficients is omitted. The claim for $\rho_{X}$ as stated above follows upon taking the ind-completion, using that $\operatorname{DM}(X)$ is compactly generated (Lemma 2.3.6).

As was recalled in the beginning of Section 2.1.2, the properties of DM listed in Synopsis 2.1.1, hold for the integral DM as well, except for (xiii) and $(\mathrm{xv})$, and provided that we replace the word 'compact' in Synopsis 2.1.1 by 'constructible'. 
Recall that, for a finite type scheme $U / S$, there is a pair of adjunctions so $\rho_{U}=$ $i_{*} \circ a^{*}$ (see [CD16, 4.5.3, 5.5.3] or [Ayo14, Section 5] under the above-mentioned condition on $S)$ :

$$
\mathrm{DM}(U) \underset{a_{*}}{\stackrel{a^{*}}{\rightleftarrows}} \mathrm{DM}(U, \mathbf{Z} / m) \underset{i_{*}}{\stackrel{i^{*}}{\cong}} \mathrm{D}_{\text {ét }}(U, \mathbf{Z} / m)
$$

The functor $a^{*}$ is obtained by applying the (derived) tensor product $-\otimes_{\mathbf{Z}} \mathbf{Z} / m$ to the coefficients, its right adjoint $a_{*}$ is the forgetful functor. The right hand equivalence is Ayoub's generalization of Suslin-Voevodsky's rigidity.

The proof of both (i) and (ii) proceeds in two steps: a first step in which $X$ is an algebraic space, and a second step in which $X$ is an Artin stack. In the first step, there is an étale covering $u: U \rightarrow X$ such that the transition maps in the Čech nerve $U^{\bullet}$ are étale maps of locally finite type $S$-schemes. In the second step, there is similarly a smooth covering by algebraic spaces. We only spell out the first step in the sequel, the second being analogous.

Throughout, we use the descent equivalence (Theorem 2.2.16):

$$
\operatorname{DM}(X) \cong \lim \mathrm{DM}^{!}\left(U^{\bullet}\right) \cong \lim \mathrm{DM}^{*}\left(U^{\bullet}\right) .
$$

At the right, the limit is taken over the same diagram, but using *-pullbacks instead. These two limits are equivalent by relative purity.

(i): Recall from [LZ11, Section 0.1] that

$$
\mathrm{D}_{\text {ét }}(X, \mathbf{Z} / m) \stackrel{\text { def }}{=} \lim \mathrm{D}_{\text {êt }}^{*}\left(U^{\bullet}, \mathbf{Z} / m\right) .
$$

Using (2.3.9), to construct $\rho_{X}$ it remains to observe that $\rho$ (on the category of $S$-schemes) is compatible with *-pullback.

(ii): Using (2.3.9) and (2.3.10) (for a smooth covering of $Y$ ) it is enough to show that (2.3.8) commutes in case $Y$ is a scheme. Consider the diagram

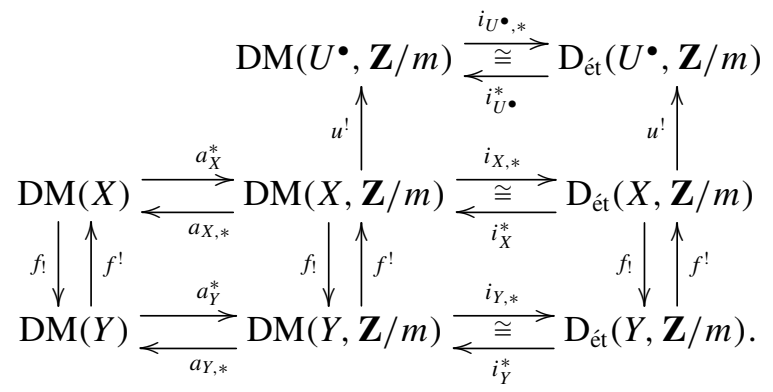

The $(-)^{*}$ and $(-)$ ! functors are the left adjoints, the others the right adjoints. To show the commutation of the large bottom rectangle involving left adjoints, it is 
enough to show the commutation of the two individual bottom squares composed of right adjoints. For the left, this follows from the natural transformation $a_{*}$ : $\mathrm{DM}^{!}(-, \mathbf{Z} / m) \rightarrow \mathrm{DM}^{!}(-, \mathbf{Z})$ of functors $\left(\mathrm{AffSch}_{S}^{\mathrm{ft}}\right)^{\text {op }} \rightarrow \mathrm{DGCat}_{\text {cont }}$, which in its turn follows from the transformation $a^{*}: \mathrm{DM}_{!}(-, \mathbf{Z}) \rightarrow \mathrm{DM}_{!}(-, \mathbf{Z} / m)$ of functors $\mathrm{AffSch}_{S}^{\mathrm{ft}} \rightarrow \mathrm{DGCat}_{\text {cont }}$ given by [Ayo14, Proposition 6.2(b)] or [CD16, Corollary 5.5.4]. To show the commutation of $f^{!}$and $i^{*}$, we use that $f^{!}: \mathrm{D}_{\text {et }}(Y, \mathbf{Z} / m) \rightarrow \mathrm{D}_{\text {ét }}(X, \mathbf{Z} / m)$ is (by functoriality of $f^{!}$) the unique functor whose composition with $u^{!}: \mathrm{D}_{\text {ét }}(X, \mathbf{Z} / m) \rightarrow \mathrm{D}_{\text {ét }}\left(U^{\bullet}, \mathbf{Z} / m\right)$ is the functor $(f \circ u)$ !. Thus, the commutativity of the lower right hand square of right adjoints is equivalent to the commutativity of the square involving the !-pullbacks along the map (of simplicial schemes) $f \circ u$. This, in turn, holds again by adjunction and the above-mentioned results in [Ayo14, CD16].

REMARK 2.3.11. It would be interesting to apply Definition 2.2.1 to the functor

$$
\mathrm{DM}_{\mathrm{et}}^{!}\left(-, \mathbf{Z} / \ell^{n}\right) \quad \text { or similarly with } \mathbf{Z}_{\ell}, \mathbf{Q}_{\ell}
$$

in order to obtain a six-functor formalism of torsion or $\ell$-adic sheaves in great generality. Another interesting question seems to investigate the relation of D-modules on prestacks as in [Ras] and $\mathrm{H}_{\mathrm{dR}}$-modules on prestacks, where $\mathrm{H}_{\mathrm{dR}}$ is the motivic ring spectrum representing de Rham cohomology.

Proposition 2.3.3 allows to conveniently define the motive of an ind-Artin stack ind-(locally of finite type) over $S$. This drops the exhaustiveness condition in [HL18, Definition 2.17] at the expense of getting only a motive in the étale topology, which is coarser than the motive in the Nisnevich topology constructed in [HL18].

Definition 2.3.12. The motive of an ind-Artin stack $f: X \rightarrow S$ in $\operatorname{IndArt}_{S}^{\mathrm{lft}}$ is defined as the object

$$
\mathrm{M}(X) \stackrel{\text { def }}{=} f_{!} f^{!} 1_{S} \in \mathrm{DM}(S) .
$$

For a presentation $X=\operatorname{colim} X_{i}$, the motive can be computed as $\mathrm{M}(X)=$ colim $\mathbf{M}\left(X_{i}\right)$, where $\mathbf{M}\left(X_{i}\right):=\left(f_{i}\right)_{!}\left(f_{i}\right)^{!} 1_{S}, f_{i}: X_{i} \rightarrow S$.

EXAMPLE 2.3.13. Let Gr be the affine Grassmannian (respectively Fl the full affine flag variety) attached to a split reductive group over the spectrum $S$ of any field, cf. Section 4 below. The standard computation of a proper $S$-scheme $X$ stratified by affine spaces $\left(X=\bigsqcup_{w \in W} \mathbf{A}_{S}^{d_{w}}\right)$ gives $\mathrm{M}(X)=\bigoplus_{w} 1\left(d_{w}\right)\left[2 d_{w}\right]$. Using the localization sequences for motives on ind-schemes (Theorem 2.4.2), this extends to ind-schemes, reproducing the computations in [Bac19, Corollary 23], 
with one summand for each affine space of a certain dimension $d_{\lambda}$ (respectively $d_{w}$ ) occurring in the stratification of $\mathrm{Gr}$ (respectively $\mathrm{Fl}$ ) by Iwahori orbits:

$$
\begin{aligned}
\mathrm{M}(\mathrm{Gr}) & =\bigoplus_{\lambda \in X_{*}(T)} 1\left(d_{\lambda}\right)\left[2 d_{\lambda}\right], \\
(\text { respectively } \mathrm{M}(\mathrm{Fl}) & \left.=\bigoplus_{w \in W} 1\left(d_{w}\right)\left[2 d_{w}\right] .\right)
\end{aligned}
$$

2.4. Motives on ind-schemes. In this section, we restrict our attention to motives on ind-schemes which are the objects of main interest in the present manuscript. Important examples are affine Grassmannians and affine flags varieties, see Section 4.3.

Let $\operatorname{IndSch}_{S}^{\mathrm{ft}}$ be the category of strict ind-schemes of ind-(finite type) over $S$. Every object $X \in \operatorname{IndSch}_{S}^{\mathrm{ft}}$ admits a presentation

$$
X=\underset{i \in I}{\operatorname{colim}} X_{i}
$$

by $S$-schemes of finite type with transition maps $t_{i, j}: X_{i} \rightarrow X_{j}$ being closed immersions for all $i \leqslant j$. The category $\operatorname{IndSch}_{S}^{\mathrm{ft}}$ is, by definition, a subcategory of the (ordinary) category of presheaves on $\mathrm{AffSch}_{S}$. We regard it as a full subcategory of PreStk ${ }_{S}$ by Remark 2.2.5. Note that $\operatorname{IndSch}_{S}^{\mathrm{ft}}$ is a full subcategory of IndArt ${ }_{S}^{\text {lft }}$, so that the results of Section 2.3 carry over to strict ind-schemes of ind-finite type over $S$. In particular, the colimit in (2.4.1) is the ordinary colimit of presheaves of sets, and each ind-scheme is an fpqc sheaf on $\mathrm{AffSch}_{S}$. Further, the computation of motives on ind-schemes reduces to the one of motives on schemes as in Corollary 2.3.4 and Lemma 2.3.6.

In order to define Whitney-Tate stratified ind-schemes (see Definition and Lemma 3.1.11), we use the six-functor formalism for motives on ind-schemes supplied by the next theorem.

THEOREM 2.4.2. Motives on ind-schemes of ind-(finite type) satisfy the properties (i)-(xii), (xiv)-(xvii) listed in Synopsis 2.1.1, with the following adjustments: (Property (xiii) does not carry over.)

- The functor $f^{*}$ is defined (and left adjoint to $f_{*}$ ) if the following condition is satisfied: there is a presentation $Y=\operatorname{colim}_{i} Y_{i}$ such that the underlying reduced locus $\left(X \times_{Y} Y_{i}\right)_{\text {red }}$ is a finite type $S$-scheme (as opposed to an ind-scheme).

- If $X$ is componentwise quasicompact, then $f^{*} 1_{S}$ is a monoidal unit, for the structural map $f: X \rightarrow S$. In general, $\operatorname{DM}(X)$ does not have a monoidal unit, cf. Example 2.4.3, but still has $\otimes$. 
- The term 'smooth' has to be replaced with 'schematic smooth' in (iii), (xii). The term 'proper' has to be replaced by 'ind-proper' in (iii), (iv). The term 'open immersion' (respectively 'closed immersion') has to be replaced with 'schematic open immersion' (respectively 'schematic closed immersion') in (iv), (ix) (respectively in (ix)). Item ( $x$ ) holds for a Cartesian diagram of ind-schemes whenever the corresponding functors are defined.

- For the descent statement in (xiv), we require a schematic h-covering $X \rightarrow Y$.

Proof. Throughout, let $f: X \rightarrow Y$ be a map in IndSch $_{S}^{\mathrm{ft}}$. Choosing suitable presentations of $X$ and $Y$, the map $f$ is a colimit of maps $f_{i}: X_{i} \rightarrow Y_{i}, i \in I$ over the same index set, cf. Appendix A.1. We write $\mathrm{ev}_{i}: \operatorname{DM}(X) \rightarrow \operatorname{DM}\left(X_{i}\right)$ and likewise for $Y_{i}$.

The functor $f^{!}$exists for any map of prestacks. It has a left adjoint $f_{\text {! }}$ by Proposition 2.3.3 which satisfies $\left(f_{i}\right)_{!} \mathrm{ev}_{i}=\mathrm{ev}_{i} f_{!}$. Here we use that the $t_{i j}: X_{i} \rightarrow$ $X_{j}$ are proper.

Tensor product and internal Hom extend by virtue of the presentation $\operatorname{DM}(X)=$ $\operatorname{colim}_{t_{*}} \operatorname{DM}\left(X_{i}\right)$ and the formulas $t_{*} M \otimes t_{*} N=t_{*}(M \otimes N)$ and $\underline{\operatorname{Hom}}\left(t_{*} M, t_{*} N\right)=$ $t_{*} \operatorname{Hom}(M, N)$, valid for a closed immersion $t$ [CD09, Theorem 2.4.50(5)].

Corollary 2.3.4 says that DM| $\left.\right|_{\text {IndSchs }_{S}^{\mathrm{ft}}}$ is also the left Kan extension of DM on $\mathrm{Sch}_{S}^{\mathrm{ft}}$, when equipped with $*$-pushforwards. We therefore obtain a functor $f_{*}$ for any map of ind-schemes. It satisfies ins ${ }_{i}\left(f_{i}\right)_{*}=f_{*}$ ins $_{i}$.

Now we construct $f^{*}$ under the above assumption. We note that $X_{\text {red }}=$ $\operatorname{colim}_{i} \tilde{X}_{i}, \tilde{X}_{i}:=\left(X \times_{Y} Y_{i}\right)_{\text {red }}$ is a presentation by finite type $S$-schemes. Using that $\operatorname{DM}\left(X \times_{Y} Y_{i}\right)=\operatorname{DM}\left(\tilde{X}_{i}\right)$ by localization, we get an equivalence

$$
\operatorname{DM}(X)=\underset{i}{\operatorname{colim}} \operatorname{DM}\left(\tilde{X}_{i}\right) \text {. }
$$

Hence, we may define $f^{*}$, noting that the $*$-pushforwards along the closed immersions given by the transition maps commute with $f^{*}$ by base change. One checks that the functor $f^{*}$ is left adjoint to $f_{*}$.

The existence of $f_{\sharp}$ for a schematic smooth map $f$ follows immediately from the case of schemes: after possibly replacing $X_{i}$ by $X \times_{Y} Y_{i}$, the map $f$ is the colimit of the smooth maps $f_{i}: X_{i} \rightarrow Y_{i}, i \in I$.

Finally, we define the subcategories $\operatorname{DM}(X)^{\mathrm{c}, \mathrm{w} \leqslant 0}$ to be the colimit (in Cat ${ }_{\infty}$ ) of the categories $\operatorname{DM}\left(X_{i}\right)^{\mathrm{c}, \mathrm{w} \leqslant 0}$ and similarly for ' $\mathrm{w} \geqslant 0$ ', using that $t_{*}=t_{\text {! }}$ is weight-exact. The existence of a weight truncation triangle $M^{\mathrm{w} \leqslant 0} \rightarrow M \rightarrow M^{\mathrm{w} \geqslant 1}$ for some compact object $M \in \operatorname{DM}(X)^{\mathrm{c}}$ follows from the above description of compact objects: $M$ is supported on some $X_{i}$, and a weight truncation triangle of $M$ in $\operatorname{DM}\left(X_{i}\right)$ gives rise to one in $\operatorname{DM}(X)$. The weight structure on compact objects extends to one on $\operatorname{DM}(X)=\operatorname{Ind}\left(\operatorname{DM}(X)^{\mathrm{c}}\right)$ by [BL16, Proposition 1.3.5]. 
EXAMPLE 2.4.3. There is no compact monoidal unit in $\operatorname{DM}\left(\mathbf{A}^{\infty}\right)$, where $\mathbf{A}^{\infty}=\operatorname{colim}\left(\mathbf{A}^{0} \stackrel{0}{\rightarrow} \mathbf{A}^{1} \stackrel{\text { id } \times 0}{\rightarrow} \mathbf{A}^{2} \ldots\right)$ is an infinite-dimensional affine space: by Lemma 2.3.6 it would be supported on some $\mathbf{A}^{i}$. However, using the complementary open immersion $u$ of the inclusion $t_{i, i+1}$, tensoring a motive supported on $\mathbf{A}^{i}$ with one of the form $u ! u^{*} M, M \in \mathrm{DM}\left(\mathbf{A}^{i+1}\right)$ gives zero. Indeed, by construction of $\otimes$ on $\operatorname{DM}\left(\mathbf{A}^{\infty}\right)$, the inclusion functors $\operatorname{DM}\left(\mathbf{A}^{i}\right) \rightarrow \operatorname{DM}\left(\mathbf{A}^{\infty}\right)$ are monoidal.

We finish this section by extending the exterior product of motives to the case of certain pro-algebraic group actions on ind-schemes. This will be needed in Section 6.

Proposition 2.4.4. For $j=1, \ldots, n$, let $X^{(j)}=\operatorname{colim}_{i} X_{i}^{(j)}$ in $\operatorname{IndSch}_{S}^{\mathrm{ft}}$ equipped with an action of a strictly pro-algebraic group $G^{(j)}=\lim G_{i}^{(j)}$ so that the $G^{(j)}$-action on $X_{i}^{(j)}$ factors over $G_{i}^{(j)}$. We assume that the kernels $\operatorname{ker}\left(G^{(j)} \rightarrow G_{i}^{(j)}\right)$ are split pro-unipotent. Then there is a functor

$$
\otimes: \operatorname{DM}\left(G^{(1)} \backslash X^{(1)}\right) \times \cdots \times \operatorname{DM}\left(G^{(n)} \backslash X^{(n)}\right) \rightarrow \operatorname{DM}\left(\prod_{j} G^{(j)} \backslash X^{(j)}\right) .
$$

Forgetting the actions of the $G^{(j)}$, and restricting to objects supported on some $X_{i_{j}}^{(j)}$, this functor agrees with the usual exterior product.

Proof. By Corollary 2.3.4, Proposition 2.2.11, and Lemma 2.2.7, there are equivalences of $\infty$-categories

$$
\begin{aligned}
& \operatorname{DM}_{G^{(j)}}\left(X^{(j)}\right)=\operatorname{colim}_{i} \mathrm{DM}_{G^{(j)}}\left(X_{i}^{(j)}\right) \\
& =\text { colim } \lim _{i} \mathrm{DM}_{i_{i^{\prime}}^{(j)}}\left(X_{i}^{(j)}\right) \\
& =\text { colim } \lim _{i} \lim \operatorname{DM}\left(\operatorname{Bar}\left(G_{i^{\prime}}^{(j)}, X_{i}^{(j)}\right)\right) \text {. }
\end{aligned}
$$

To define a functor with the asserted properties it is therefore enough to observe that $\otimes$ commutes with the !-pushforward along the closed immersions used to form the strict ind-schemes $X^{(j)}$ and then that $\nabla$ commutes with the !-pullback along the smooth action and projection maps in the bar construction $\operatorname{Bar}\left(G_{i^{\prime}}^{(j)}, X_{i}^{(j)}\right)$.

\section{Stratified Tate motives}

In this section, we discuss stratified Tate motives, that is, motives which are Tate motives on each stratum of a given stratification. We work in the generality 
of Tate motives on stratified ind-schemes relative to a base scheme, which extends the work of Soergel and Wendt [SW18, Sections 3-4].

Notation 3.0.1. Our base scheme $S$ is as in Notation 2.0.1. By convention, all ind-schemes are strict $S$-ind-schemes of ind-finite type. Recall from Section A.1 that we only consider ind-schemes indexed by countable index sets.

\subsection{Definitions and elementary construction principles.}

DEFINITION 3.1.1. (i) A stratified ind-scheme is a map of ind-schemes

$$
\iota: X^{+}=\bigsqcup_{w \in W} X_{w} \rightarrow X
$$

such that $\iota$ is bijective on the underlying sets, each stratum $X_{w}$ is a scheme, the restriction to each stratum $\iota_{X_{w}}$ is representable by a quasicompact immersion and the topological closure of each stratum $\iota\left(X_{w}\right)$ is a union of strata.

(ii) A map of stratified ind-schemes is a commutative diagram of (ind-)schemes

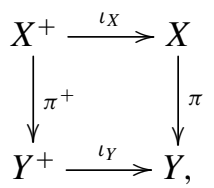

where $\pi$ is a schematic map of finite type, and $\pi^{+}$maps each stratum in $X^{+}$ into a stratum in $Y^{+}$. The latter condition is automatically satisfied whenever the strata are connected.

REMARK 3.1.3. (i) If $X$ happens to be a finite type $S$-scheme, then $W$ is necessarily finite. For an ind-scheme, $W$ may be countably infinite. However, all the $X_{w}$ are necessarily of finite type.

(ii) By the localization sequence in Synopsis 2.1.1(ix), the category $\operatorname{DM}(X)$ only depends on the underlying reduced ind-scheme structure. After possibly replacing $X$ (respectively $X^{+}$) by their reduced sub-ind-schemes, we may and do assume $X$ and $X^{+}$to be reduced.

(iii) We do not in general assume the strata $X^{+}=\bigsqcup_{w \in W} X_{w}$ to be regular (or smooth over $S$ ).

(iv) Let $\iota: X^{+}=\bigsqcup_{w \in W} X_{w} \rightarrow X$ be a stratified ind-scheme. Let $X=\operatorname{colim}_{i} X_{i}$ be any ind-presentation. For each $w \in W$, the map $\left.\iota\right|_{X_{w}}: X_{w} \rightarrow X$ factors 
as $X_{w} \rightarrow X_{i} \subset X$ for some $i \gg 0$ because $X_{w}$ is quasicompact (being a finite type $S$-scheme). Hence, the scheme-theoretic image $\overline{X_{w}} \subset X_{i}$ of $\iota_{X_{w}}$ is a separated $S$-scheme of finite type, and its underlying topological space agrees with the topological closure of $\iota\left(X_{w}\right)$, cf. [Sta17, Tag 01R8]. Hence, the base change map ${\overline{X_{w}}}^{+}:=X^{+} \times_{X} \overline{X_{w}} \rightarrow \overline{X_{w}}$ is a stratified scheme, and $\bar{X}_{w}^{+}=\bigsqcup_{v \leqslant w} X_{v}$ is an (automatically finite) union of strata. There is a presentation

$$
X=\underset{w \in W}{\operatorname{colim}} \overline{X_{w}},
$$

where $W$ is partially ordered by the closure relations of the strata. We can think about $\iota: X^{+} \rightarrow X$ as being the colimit of the stratified schemes $\bar{X}_{w}^{+}=$ $\bigsqcup_{v \leqslant w} X_{v} \rightarrow \overline{X_{w}}$.

All stratified (ind-)schemes we encounter in Section 5 are cellular in the following sense:

DEFINITION 3.1.5. An $S$-cell is an $S$-scheme isomorphic to $\mathbb{V}(\mathcal{E}) \times \prod_{i=1}^{r} \mathbb{V}^{\times}\left(\mathcal{E}_{i}\right)$ for some vector bundles $\mathcal{E}, \mathcal{E}_{i}$ on $S$ (see (A.4) for notation). A cellular $S$-scheme $X$ is a separated $S$-scheme of finite type which is smooth and admits a stratification into cells. A cellular stratified $S$-ind-scheme $\iota: X^{+}=\bigsqcup_{w \in W} X_{w} \rightarrow X$ is a stratified $S$-ind-scheme where $X_{w}$ is a cellular $S$-scheme for every $w \in W$.

EXAMPLE 3.1.6. The affine Grassmannian $\mathrm{Gr}_{G}$ for a split reductive group scheme $G$ over $S$, equipped with its stratification by $L^{+} G$-orbits, and more general ind-schemes are shown to be cellular in Section 4.3.

Our notion of cellularity is less restrictive than the one in [DI05], say, which requires a stratification by affine spaces. In particular any split reductive group $G$ over $S$ is cellular in our sense by means of the Bruhat stratification.

We just write $\iota: X^{+} \rightarrow X$ or even $X$ whenever $X^{+}=\bigsqcup_{w \in W} X_{w}$ or $\iota$ are clear from the context, and likewise for stratified maps. Given a stratified map (3.1.2), we have a commutative diagram of ind-schemes

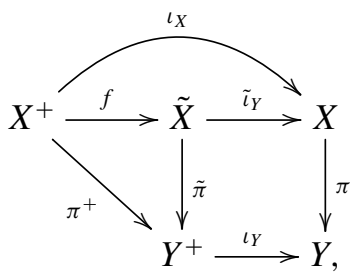


where the square is cartesian. It is easily checked that $\tilde{\iota}_{Y}: \tilde{X}=\bigsqcup_{w \in W_{Y}}\left(Y_{w} \times_{Y} X\right)$ $\rightarrow X$ and $f: X^{+}=\bigsqcup_{w \in W_{X}} X_{w} \rightarrow \tilde{X}$ are again stratifications. Thus, a stratified map amounts to possibly refining the preimage of the stratification on $Y$.

Definition 3.1.8. For a scheme $X$ of the form $X=\bigsqcup_{w \in W} X_{w}$, with $X_{w} \rightarrow S$ of finite type, the category of Tate motives

\section{$\operatorname{DTM}(X) \subset \operatorname{DM}(X)$}

is the stable cocomplete sub- $\infty$-category generated by the objects $1_{X}(n)$, for $n \in \mathbf{Z}$.

A map $\pi: X \rightarrow Y$ between such ind-schemes is called a Tate map if $\pi_{*} 1_{X} \in \operatorname{DTM}(Y)$. This holds true if and only if the adjunction (2.1.2) restricts to an adjunction

$$
\pi^{*}: \operatorname{DTM}(Y) \rightleftarrows \operatorname{DTM}(X): \pi_{*} .
$$

REMARK 3.1.9. By definition, $\operatorname{DTM}(X)$ is large in the sense that it contains arbitrary coproducts. This implies in particular that it is idempotent complete, that is, stable under taking direct summands. For example, if $\iota: X_{w} \rightarrow X$ is the inclusion of a connected component of $X$, then $\operatorname{DTM}(X)$ contains $\iota_{*} 1_{X_{w}}(n)$ because it is a direct summand of $1_{X}(n)$. We often apply this remark in the case where $X$ is the disjoint union of strata in some scheme. If $X$ has infinitely many (finite type) connected components $X_{w}, 1_{X}$ is not compact. Indeed, by Lemma 2.3.6 motive is compact if and only if its support is contained in finitely many $X_{w}$ 's and is a compact object in the usual sense there.

EXAMPLE 3.1.10. For any vector bundles $\mathcal{E}, \mathcal{E}_{1}, \ldots, \mathcal{E}_{b}$ on $Y$, the projection $\pi: X:=\mathbb{V}(\mathcal{E}) \times \prod_{i=1}^{b} \mathbb{V}^{\times}\left(\mathcal{E}_{i}\right) \rightarrow Y$ (see (A.4) for notation) is a Tate map, by the definition of Tate objects, homotopy invariance and localization [Dég08, 4.20].

The following condition, introduced by Soergel and Wendt [SW18, Section 4] for Tate motives on stratified schemes (as opposed to ind-schemes), ensures a well-behaved notion of stratified Tate motives:

Definition And Lemma 3.1.11. Let $\iota: X^{+}=\bigsqcup_{w \in W} X_{w} \rightarrow X$ be a stratified ind-scheme. The following are equivalent:

(i) $\iota^{*} \iota_{*} 1_{X^{+}} \in \operatorname{DTM}\left(X^{+}\right)$;

(ii) $\iota ! \iota_{!} 1_{X^{+}} \in \operatorname{DTM}\left(X^{+}\right)$.

If either (i) or (ii) holds true, then $\iota: X^{+} \rightarrow X$ is called a Whitney-Tate stratification. In this case, the following subcategories (a)-(d) of $\operatorname{DM}(X)$ are all the same: 
(a) $\left\langle\iota_{w, *} 1_{X_{w}}(n) ; w \in W\right\rangle$, where $\iota_{w}:=\left.\iota\right|_{X_{w}}: X_{w} \rightarrow X$ denotes the inclusion of a stratum, and ' $\langle-\rangle$ ' denotes the stable sub- $\infty$-category generated under arbitrary (homotopy) colimits, suspensions and desuspensions. (In the language of triangulated categories, this corresponds to the closure under arbitrary shifts, extensions, and coproducts.)

(b) $\left\langle\iota_{w, !} 1_{X_{w}}(n) ; w \in W\right\rangle$,

(c) $\left\{M \in \mathrm{DM}(X), \iota^{!} M \in \mathrm{DTM}\left(X^{+}\right)\right\}$,

(d) $\left\{M \in \operatorname{DM}(X), \iota^{*} M \in \operatorname{DTM}\left(X^{+}\right)\right\}$.

This category is denoted $\operatorname{DTM}\left(X, X^{+}\right)$or just $\operatorname{DTM}(X)$ if the stratification is clear from the context.

Proof. For finite type schemes $X$ with regular $X^{+}$, this is due to [SW18, Section 4]. The regularity assumption is unnecessary, by replacing the usage of Verdier duality by localization arguments such as the cofiber sequence $i^{!} \rightarrow i^{*} \rightarrow$ $i^{*} j_{*} j^{*}$ (obtained by applying $i^{*}$ to (2.1.4) for two complementary closed and open embeddings $i$ and $j$. The extension from finite type schemes to ind-schemes is formal, using the following remark: write $X=\operatorname{colim}_{w} \bar{X}_{w}$ as in (3.1.4). Condition (i) is equivalent to the condition (i) for all $w \in W: \iota_{w}^{*} \iota_{*} 1 \in \operatorname{DTM}\left(X_{w}\right)$. Using that the closure $\overline{X_{w}}$ in $X$ consists only of finitely many strata, Condition (i) $)_{\mathrm{w}}$ for $X$ is equivalent to Condition (i) w $_{\mathrm{w}}$ for $\overline{X_{w}}$. Similarly for (ii), which reduces the equivalence (i) $\Leftrightarrow$ (ii) to the case of schemes, and accomplishes the proof. The agreement of (a)-(d) can also be reduced to the case of schemes in a similar manner.

REMARK 3.1.12. Following up Remark 3.1.9, DTM $\left(X, X^{+}\right)$is again cocomplete. The consideration of these large categories is merely a matter of convenience. We could instead consider its subcategory of compact objects, which by Lemma 2.3.6 consists precisely of those Tate motives (in the above sense) whose support is contained in finitely many strata and is compact there.

EXAMPLE 3.1.13. Let $k$ be a field, and let $G$ be a split reductive $k$-group with Borel subgroup $B$. Then the Bruhat stratification by $B$-orbits on $G / B$ is a cellular Whitney-Tate stratification by [SW18, Proposition 4.10]. We reprove and extend this statement to the case of partial affine flag varieties in Theorem 5.1.1.

REMARK 3.1.14. Let $X^{+} \rightarrow X$ be a Whitney-Tate stratified ind-scheme with $X^{+}$being a regular scheme. Then $\operatorname{DTM}(X) \subset \operatorname{DM}(X)$ is stable under the dualising functor D (Synopsis 2.1.1(viii)). Indeed, $\mathrm{D}\left(\iota^{*} \iota_{*} 1\right)=\iota^{!} \iota_{!} \mathrm{D}(1)=$ $\iota^{!} \iota ! 1\left(\operatorname{dim} X^{+}\right)\left[2 \operatorname{dim} X^{+}\right]$where $\operatorname{dim} X^{+}:\left|X^{+}\right| \rightarrow \mathbf{Z}_{\geqslant 0}$ is viewed as a locally constant function. 
Definition 3.1.15. A stratified map $\pi:\left(X, X^{+}\right) \rightarrow\left(Y, Y^{+}\right)$of Whitney-Tate stratified ind-schemes is a Whitney-Tate map if (2.1.2) restricts to an adjunction

$$
\pi^{*}: \operatorname{DTM}\left(Y, Y^{+}\right) \rightleftarrows \operatorname{DTM}\left(X, X^{+}\right): \pi_{*} .
$$

REMARK 3.1.16. (i) Definition 3.1.15 is in effect only a condition on $\pi_{*}$ since $\pi^{*}$ preserves Tate motives for any stratified map: using the notation of (3.1.7), $\iota_{X}^{*} \pi^{*} \iota_{Y, !} 1_{Y^{+}}=f^{*} \tilde{\iota}_{Y}^{*} \tilde{l}_{Y, !} 1_{\tilde{X}}$. By localization, $1_{\tilde{X}}$ lies in the smallest subcategory generated by $f_{\tilde{\tau}} 1$ under extensions and retracts. Indeed, each connected component of $\tilde{X}$ is of finite type and is therefore stratified by finitely many $X_{w}$. We conclude the claim from the localization sequence (2.1.5). Hence the above motive is obtained by extensions and direct summands from $\iota_{X}^{*} \iota_{X, !} 1 \in \operatorname{DTM}\left(X^{+}\right)$.

(ii) In addition, if $X^{+}$and $Y^{+}$are regular, then the Whitney-Tate condition on $\pi$ is equivalent to the existence of an adjunction

$$
\pi_{!}: \operatorname{DTM}\left(X, X^{+}\right) \rightleftarrows \operatorname{DTM}\left(Y, Y^{+}\right): \pi^{!} .
$$

This follows from Remark 3.1.14. Finally, if in addition $\pi$ is smooth, then the Whitney-Tate condition can also be expressed using $\pi_{\sharp}$ (using the equivalence $\pi_{\sharp}=\pi_{!}(d)[2 d], d$ being the relative dimension of $\left.\pi\right)$.

EXAMPLE 3.1.17. (i) If a schematic smooth map $\pi: X \rightarrow Y$ of finite type and a Whitney-Tate stratification $\iota_{Y}: Y^{+} \rightarrow Y$ is given, then the preimage stratification $\iota_{X}: X^{+}:=X \times_{Y} Y^{+} \rightarrow X$ is again Whitney-Tate. Indeed, $\iota_{X}^{*} \iota_{X, *} 1=\pi^{+, *} \iota_{Y}^{*} \iota_{Y, *} 1$ using smooth base change, which is a Tate motive since $Y$ is Whitney-Tate.

(ii) If, in addition, $\pi^{+}: X^{+} \rightarrow Y^{+}$is a Tate map, then $\pi$ is a Whitney-Tate map.

The following lemmas give relations between (partial) Whitney-Tate properties of source and target of a proper map. They will be used to show that partial affine flag varieties are Whitney-Tate, cf. Section 5.

LEMmA 3.1.18. Let $\pi: X \rightarrow Y$ be a map of stratified ind-schemes such that $\pi^{+}$ is a Tate map. We assume that either $\pi^{+}$is smooth or that $X^{+}$and $Y^{+}$are both regular. (We do not assume the stratifications on $X$ or $Y$ are Whitney-Tate.) If $M \in \mathrm{DM}(X)$ is such that $\iota_{X}^{!} M \in \operatorname{DTM}\left(X^{+}\right)$, then $\iota_{X}^{!} \pi^{!} \pi_{*} M \in \operatorname{DTM}\left(X^{+}\right)$.

Proof. We use the notation of (3.1.7) and compute $\iota_{X}^{!} \pi^{!} \pi_{*} M=\left(\pi^{+}\right) ! \tilde{\pi}_{*} \tilde{l}_{Y}^{\prime} M$. Any $N \in \operatorname{DTM}(\tilde{X})$ lies in the subcategory generated by the summands (corresponding 
to the connected components of $\left.X^{+}\right)$of $f_{*} f^{!} N$ by localization. We may thus consider $\left(\pi^{+}\right) ! \tilde{\pi}_{*} f_{*} f^{!} \tilde{l}_{Y}^{!} M=\left(\pi^{+}\right)^{!} \pi_{*}^{+} \iota_{X}^{!} M$ instead. This is a Tate motive by one of the assumptions.

LEMma 3.1.19. Let $\pi: X \rightarrow Y$ be a proper map of stratified ind-schemes such that the map $\pi^{+}: X^{+} \rightarrow Y^{+}$has a section $s^{+}: Y^{+} \rightarrow X^{+}$. Assume that $s^{+}$is an open and closed immersion which identifies the strata of $Y^{+}$with some strata of $X^{+}$. Further, assume that $X$ is Whitney-Tate, and that $\pi^{+}$is a Tate map between regular schemes. Then $Y$ is also Whitney-Tate, and $\pi$ is a Whitney-Tate map.

Proof. We have to show that $\iota_{Y}^{*} \iota_{Y, *} 1 \in \mathrm{DTM}\left(Y^{+}\right)$. Using the notation of (3.1.7), we compute

$$
\begin{aligned}
\iota_{Y}^{*} \iota_{Y, *} 1 & =\iota_{Y}^{*} \pi_{*} \tilde{l}_{Y, *} f_{*} s_{*}^{+} 1 \\
& =\tilde{\pi}_{*} \tilde{l}_{Y}^{*} \tilde{\iota}_{Y, *} f_{*} s_{*}^{+} 1 \quad \text { (proper base change) } .
\end{aligned}
$$

The map $s^{+}$identifies, by assumption, the strata of $Y^{+}$with strata of $X^{+}$, and therefore $s_{*}^{+} 1 \in \operatorname{DTM}\left(X^{+}\right)$. Let $M \in \operatorname{DM}(\tilde{X})^{\mathrm{c}}$ be any compact object. The localization property of motives (Synopsis 2.1.1(ix), together with an induction on the finite number of strata on which $M$ is supported) implies that $\tilde{\pi}_{!} M$ is an extension of direct summands of $\pi_{1}^{+} f^{*} M$. It is thus enough to show that $\pi_{!}^{+} f^{*} \tilde{l}_{Y}^{*} \tilde{l}_{Y, *} f_{*} s_{*}^{+} 1$ is a Tate motive. This holds since $\pi_{!}^{+}$and $\iota_{X}^{*} \iota_{X, *}=f^{*} \tilde{l}_{Y}^{*} \tilde{l}_{Y, *} f_{*}$ preserve Tate motives by assumption and Remark 3.1.16. This shows that $Y$ is Whitney-Tate.

In order to see that $\pi$ is Whitney-Tate, we use that $\pi_{*} \tilde{\imath}_{Y, *} f_{*} 1=\pi_{*}^{+} \iota_{Y, *} 1$ is a Tate motive on $Y$ because $\pi^{+}$and $\iota_{Y}$ are both Tate maps by assumption and the previous step, respectively. This proves the lemma.

LEMMA 3.1.20. Let $\pi: X \rightarrow Y$ be a smooth stratified map of stratified WhitneyTate ind-schemes such that $X^{+}$and $Y^{+}$are regular, $\pi^{+}$is a Tate map, and such that in the notation in (3.1.7) the adjunction map $\tilde{\pi}_{\sharp} 1 \rightarrow 1$ admits a section (this holds true if $\tilde{\pi}$ or, a fortiori, $\pi^{+}$admits a section). Then $\pi^{*}$ preserves and detects Tate motives in the following sense: for $M \in \mathrm{DM}(Y)$ one has

$$
\pi^{*} M \in \operatorname{DTM}(X) \Leftrightarrow M \in \operatorname{DTM}(Y) .
$$

Proof. The implication $\Leftarrow$ follows from Remark 3.1.16. Conversely, we use the projection formula [CD09, 1.1.26] (extended to motives over ind-schemes as discussed in Section 2.4) and smooth base change to see that the adjunction map

$$
\tilde{\pi}_{\sharp} \tilde{\pi}^{*} 1 \otimes \iota_{Y}^{*} M=\tilde{\pi}_{\sharp} \tilde{\pi}^{*} \iota_{Y}^{*} M=\iota_{Y}^{*} \pi_{\sharp} \pi^{*} M \rightarrow \iota_{Y}^{*} M
$$


admits a section by assumption. Since $\pi_{\sharp}$ preserves stratified Tate motives (by assumption and Remark 3.1.16(ii)), we are done.

DEFINITION 3.1.21. Suppose an ordinary presheaf of $S$-groups $G$ acts on a stratified ind-scheme $X$. If the stratification on $X$ is Whitney-Tate, then we define the category $\operatorname{DTM}_{G}(X)$ of $G$-equivariant stratified Tate motives as the homotopy pullback

$$
\operatorname{DTM}_{G}(X) \stackrel{\text { def }}{=} \operatorname{DTM}(X) \times_{\operatorname{DM}(X)} \operatorname{DM}(G \backslash X),
$$

that is, as the full subcategory of $\operatorname{DM}(G \backslash X)$ whose underlying object in $\operatorname{DM}(X)$ is in $\operatorname{DTM}(X)$.

REMARK 3.1.22. The category $\operatorname{DTM}_{G}(X)$ is defined even if the $G$-action on $X$ does not preserve the stratification. For example, we can consider the category $\mathrm{DTM}_{L^{+} G}\left(\mathrm{Gr}_{G}\right)$ where $\mathrm{Gr}_{G}$ is equipped with the stratification into either $L^{+} G$-orbits or Iwahori orbits. In the latter case, the $L^{+} G$-action does not preserve the strata, but it turns out that the resulting categories are the same.

The notation $\operatorname{DTM}_{G}(X)$ (as opposed to $\operatorname{DTM}(G \backslash X)$ ) highlights the fact that the category of stratified Tate motives does depend on the given presentation of the prestack quotient $G \backslash X$. While descent does hold for DM, it does not hold for DTM: being a Tate motive is a property of motives which does not in general descend. However, here is a sufficient condition which ensures such a descent behavior.

Proposition 3.1.23. Let $H \subset G$ be an inclusion of ordinary $\tau$-sheaves of $S$-groups where $\tau$ is a Grothendieck topology as in Theorem 2.2.16. Let $X:=$ $(G / H)^{\tau}$ be the quotient of $\tau$-sheaves which we assume to be a smooth finite type $S$-scheme. We suppose X carries a Whitney-Tate stratification such that the base point $S \rightarrow X$ is a stratified map, that is, factors through $X^{+}$. Then the equivalence $\mathrm{DM}_{G}(X)=\mathrm{DM}_{H}(S)$ established in Lemma 2.2.21 restricts to an equivalence

$$
\operatorname{DTM}_{G}(X) \stackrel{\simeq}{\longrightarrow} \operatorname{DTM}_{H}(S)
$$

In particular, the left hand category is insensitive to the choice of the stratification (under the above assumption).

Proof. We have a commutative diagram of prestacks, where the vertical maps are the standard quotient maps and $\pi$ is the structural map of $S$, and $\sim$ indicates that the map $\gamma$ becomes an isomorphism after $\tau$-sheafification so that $\gamma$ ! is an 
equivalence by Theorem 2.2.16:

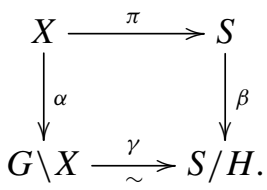

We have the following commutative diagram of $\infty$-categories:

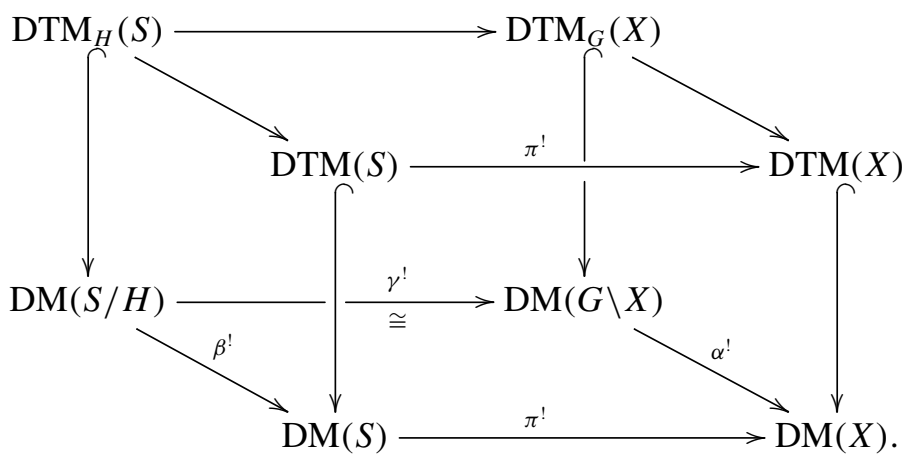

The left and right face is cartesian by definition of equivariant Tate motives. The front face is cartesian by Lemma 3.1.20 (which uses the assumption on the stratified section). We conclude that the back square is cartesian, so we get our claim.

EXAMPLE 3.1.25. The existence of a section in the above proposition is crucial: consider a finite Galois extension $K / k$ with Galois group $G$ and let $X:=\operatorname{Spec} K \stackrel{\pi}{\rightarrow} \operatorname{Spec} k=: S$. Then $(G \backslash X)^{\text {ét }}=S$, but the natural functor $\operatorname{DTM}(S) \rightarrow \operatorname{DTM}_{G}(X)$ (whose composite with the forgetful functor to $\operatorname{DTM}(X)$ is just $\pi^{!}$) is fully faithful, but not essentially surjective, since $1 \in \operatorname{DTM}_{G}(X)$ is not in the image. In fact, $\mathrm{DTM}_{G}(X)$ can be identified with the category $M$ of Artin-Tate motives on $S$ such that $\pi^{!} M$ is a Tate motive.

DEFINITION 3.1.26. A stratified $G$-action of an ordinary group presheaf $G$ on a stratified ind-scheme $\left(X, X^{+}\right)$is an action $G \times{ }_{S} X \rightarrow X$ which restricts to an action (necessarily in a unique way) $G \times_{S} X^{+} \rightarrow X^{+}$. If $G$ is algebraic and fiberwise connected, this is equivalent to requiring that each stratum $X_{w}$ is $G$-stable.

Let $G=\lim _{i \geqslant 0} G_{i}$ be strictly pro-algebraic, cf. Section A.2. By taking suitable unions of $\overline{X_{w}}, w \in W$ there exists a presentation $X=\operatorname{colim}_{i \geqslant 0} X_{i}$ with the 
following properties (cf. Lemma A.2): for every $i \geqslant 0$, the $G$-action on $X_{i}$ factors through $G_{i}$, and the map $\iota_{i}: X^{+} \times_{X} X_{i} \rightarrow X_{i}$ is a stratified scheme of the form $X_{i}^{+}=\bigsqcup_{w \in W_{i}} X_{w}$ for a suitable finite subset $W_{i} \subset W$.

Proposition 3.1.27. Let $X=\operatorname{colim}_{i} X_{i}$ be a stratified Whitney-Tate indscheme equipped with a stratified $G$-action of a strictly pro-algebraic group $G=\lim G_{i}$ so that the $G$-action on $X_{i}$ factors over $G_{i}$. We assume that the kernels $\operatorname{ker}\left(G \rightarrow G_{i}\right)$ are split pro-unipotent. Then there are equivalences of $\infty$-categories

$$
\begin{aligned}
\operatorname{DTM}_{G}(X) & =\lim _{i} \operatorname{DTM}_{G}\left(X_{i}\right) \\
& =\lim _{i} \lim _{j \geqslant i} \operatorname{DTM}_{G_{j}}\left(X_{i}\right) \\
& =\lim _{i} \lim _{j \geqslant i} \lim \operatorname{DTM}^{!}\left(\operatorname{Bar}\left(G_{j}, X_{i}\right)\right),
\end{aligned}
$$

where all the limits are formed by !-pullbacks.

Proof. The first equivalence follows from the definitions and the commutation of homotopy pullbacks of $\infty$-categories with limits. The second equivalence follows from Proposition 2.2.11. (The restriction functors $\operatorname{DTM}_{G_{j}}\left(X_{i}\right) \rightarrow \mathrm{DTM}_{G_{j^{\prime}}}\left(X_{i}\right)$ are equivalences for all $j^{\prime} \geqslant j \geqslant i$, but we keep them in order to be able to take the colimit over $i$.) To simplify notation, we now write $G$ for $G_{j}$ and $X$ for $X_{i}$. For each $n \geqslant 0$, the stratification $G^{n} \times{ }_{S} X^{+} \rightarrow G^{n} \times{ }_{S} X$ obtained by pulling back the one on $X$ is Whitney-Tate by Example 3.1.17(i), so the categories DTM $\left(G^{n} \times{ }_{S} X\right)$ are well-defined. In addition, all maps appearing in $\operatorname{Bar}(G, X)$ are maps of stratified schemes since the action (respectively projection) map $G \times{ }_{S} X \rightarrow X$ is smooth and is stratified by assumption (respectively construction). Hence, the !-pullback along these maps preserves the subcategories $\operatorname{DTM}\left(G^{n} \times{ }_{S} X\right)$ by Remark 3.1.16(i). Thus DTM! $(\operatorname{Bar}(G, X))$ is well-defined.

By Lemma 2.2.12, there is an equivalence of $\infty$-categories

$$
\lim \operatorname{DTM}^{!}(\operatorname{Bar}(G, X))=\lim \left(\operatorname{DTM}(X) \times_{\operatorname{DM}(X)} \operatorname{DM}^{!}(\operatorname{Bar}(G, X))\right) .
$$

Indeed, in both limits, the category at the 0 -vertex is $\operatorname{DTM}(X)$. The right hand side computes $\operatorname{DTM}_{G}(X)$ by the commutation of limits with pullbacks.

\subsection{Stratified mixed Tate motives.}

Notation 3.2.1. Throughout Section 3.2, $S$ is a scheme satisfying the conditions in Notation 2.0.1, and moreover satisfies the Beilinson-Soulé vanishing conjecture as in (3.2.2). 
Moreover, $\iota: X^{+} \rightarrow X$ is a cellular Whitney-Tate stratified ind-scheme in the sense of Definitions 3.1.5, 3.1.11.

Recall from [Lur17, Proposition 1.2.1.16], that a $t$-structure amounts to giving a full subcategory $\mathcal{C}{ }^{\geqslant} \subset \mathcal{C}$ which is closed under extensions and such that the inclusion functor incl admits a left adjoint $\tau \geqslant 0$. An exact functor $F$ between two stable categories with $t$-structures is left t-exact if it preserves the ' $\geqslant 0$ '-subcategories. The subcategory $\mathcal{C}^{\leqslant-1}:=\operatorname{ker} \tau^{\geqslant 0}=\left\{M \in \mathcal{C}, \tau^{\geqslant 0} M=0\right\}$ agrees with the subcategory spanned by the objects $M$ such that the mapping space $\operatorname{Hom}_{\mathcal{C}}(M, \mathcal{C} \geqslant 0)$ is contractible. Then $F$ is called right t-exact if it preserves the ' $\leqslant-1$ ' subcategories. A functor is $t$-exact if it is both rightand left t-exact.

3.2.1. The motivic t-structure. In this section we introduce the motivic $t$-structure and use it to cut down the category $\operatorname{DTM}(X)$, for a Whitney-Tate stratified scheme $X$, to the abelian category $\operatorname{MTM}(X)$ of stratified mixed Tate motives. The consideration of Tate motives in this paper has two reasons:

(i) The motivic $t$-structure on $\operatorname{DM}(X)$, predicted by the 'standard' conjectures seems to be out of reach at the moment. By contrast, this $t$-structure is known to exist for the subcategory $\operatorname{DTM}(X) \subset \operatorname{DM}(X)$, under some (rather severe) restrictions on the base scheme $X$, see Lemma 3.2.4.

(ii) The realization functor is known to be conservative on Tate motives, see Lemma 3.2.8.

Recall that the Beilinson-Soulé vanishing conjecture holds for $S$ if

$$
\operatorname{Hom}_{\operatorname{DM}(S)}(1,1(n)[m])=K_{2 n-m}(S)_{\mathbf{Q}}^{(n)} \stackrel{!}{=} 0
$$

holds for $m<0$ and also for $m=0$ and $n \neq 0$.

EXAMPLE 3.2.3. The Beilinson-Soulé conjecture is known by Quillen's, Borel's, and Harder's work, for $S$ being the spectrum of a finite field, a number field or localizations of its ring of integers, and finally for a smooth curve over a finite or its function field. Since $K$-theory commutes with filtered colimits, it also holds for filtered colimits of such rings; for example, for the algebraic closures $\overline{\mathbf{F}}_{p}$ or $\overline{\mathbf{Q}}$. See the references cited in [SW18, Remark 3.10] and [DG05, 1.6].

The following lemma is a mild extension of [SW18, Theorem 3.7]: we consider infinite disjoint unions of cellular schemes, and we also allow noncompact objects. The proof is the same as there, using in addition that a $t$-structure on a stable $\infty$-category yields a $t$-structure on its ind-completion. 
LEMMA 3.2.4. With $S$ as in Notation 3.2.1, every cellular $S$-scheme also satisfies the Beilinson-Soulé vanishing conjecture. If $X^{+}=\bigsqcup_{w \in W} X_{w}$ is a possibly infinite disjoint union of cellular schemes, then one has:

(i) The category $\operatorname{DTM}\left(X^{+}\right)$carries a unique t-structure such that both the dualising functor $\mathrm{D}_{X^{+}}$and the twisting functors $M \mapsto M(n), n \in \mathbf{Z}$ are exact. Equivalently, the heart is the subcategory of $\mathrm{DM}(X)$ which is generated by means of extensions and arbitrary coproducts by the objects

$$
1_{X_{w}}(n)\left[d_{w}\right], \quad(n \in \mathbf{Z}),
$$

where $d_{w}:\left|X_{w}\right| \rightarrow \mathbf{Z}_{\geqslant 0}$ is the relative dimension of $X_{w} \rightarrow S$ viewed as a locally constant function. The $t$-structure is called the motivic $t$-structure, and its heart $\operatorname{MTM}\left(X^{+}\right)$is the $\mathbf{Q}$-linear abelian category of mixed Tate motives. This $t$-structure restricts to one on the subcategory $\operatorname{DTM}(X)^{\mathrm{c}}$ of compact objects.

(ii) The category $\mathrm{DTM}\left(X^{+}\right)$has a weight structure whose heart is generated-by means of coproducts and extensions - by the objects $1(n)[2 n], n \in \mathbf{Z}$.

(iii) The $t$-structure is transversal to the weight structure in the sense of [Bon12, Section 1.2]. In particular, any compact object $M \in \operatorname{MTM}\left(X^{+}\right)^{\mathrm{c}}$ has a functorial weight filtration, that is, a finite sequence of subobjects

$$
0=M_{0} \subset M_{1} \subset \cdots \subset M_{n}=M,
$$

with $M_{i} \in \operatorname{MTM}\left(X^{+}\right)^{\mathrm{c}}$ such that $M_{i} / M_{i-1}$ is a finite direct sum of $1_{X_{w}}\left[d_{w}\right]\left(\left(d_{w}-i\right) / 2\right)$ (if $d_{w}-i$ is odd, this object is to be interpreted as 0$)$ for $w \in W$.

The $t$-structures on Tate motives on individual strata can be glued together:

COROLlARY 3.2.6. The category $\operatorname{DTM}\left(X, X^{+}\right)$carries the motivic $t$-structure which is glued from the motivic $t$-structures on the strata $X^{+}=\bigsqcup_{w \in W} X_{w}$ (cf. Lemma 3.2.4). That is, the motivic $t$-structure satisfies

$$
\begin{aligned}
\operatorname{DTM}(X)^{\leqslant 0} & =\left\{M \in \operatorname{DTM}(X), \iota^{*} M \in \operatorname{DTM}\left(X^{+}\right)^{\leqslant 0}\right\} \\
& =\left\{M, \iota_{w}^{*} M \in \operatorname{DTM}\left(X_{w}^{+}\right)^{\leqslant 0} \text { for all } w\right\}, \\
\operatorname{DTM}(X)^{\geqslant 0} & =\left\{M \in \operatorname{DTM}(X), \iota^{!} M \in \operatorname{DTM}\left(X^{+}\right)^{\geqslant 0}\right\} \\
& =\left\{M, \iota_{w}^{*} M \in \operatorname{DTM}\left(X_{w}^{+}\right)^{\geqslant 0} \text { for all } w\right\} .
\end{aligned}
$$

The heart $\operatorname{MTM}(X)=\operatorname{MTM}\left(X, X^{+}\right)$of the motivic $t$-structure is the category of (stratified) mixed Tate motives. It is abelian and $\mathbf{Q}$-linear. This $t$-structure is 
conservative (a.k.a. nondegenerate): an object $M \in \mathrm{DTM}(X)$ is 0 if and only if ${ }^{\mathrm{m}} \mathrm{H}^{i} M=0$ for all $i \in \mathbf{Z}$ (truncation with respect to the motivic $t$-structure). This $t$-structure restricts to one on the subcategory $\mathrm{DTM}(X)^{\mathrm{c}}$ of compact objects.

Proof. As in [SW18, Theorem 10.3], we can glue the individual motivic $t$ structures on the strata $X_{w}$, using [BBD82, Theorem 1.4.10]. The conservativity of the $t$-structure is immediate from the fact that $M=0$ if and only if $\iota_{w}^{*} M=0$ for all $w \in W$ if and only if $\iota_{w}^{!} M=0$ for all $w \in W$ and the conservativity of the $t$-structure on DTM $\left(X_{w}\right)$.

REMARK 3.2.7. In the proof of Proposition 3.2.20, we also use the classical $t$-structure on $\operatorname{DTM}(X)$. The name 'classical' refers to the analogy with the classical (respectively standard) $t$-structure on sheaves. For an individual stratum $X_{w}, w \in W$, we declare the classical $t$-structure on $\operatorname{DTM}\left(X_{w}\right)$ to be the unique $t$-structure such that $1_{X_{w}}(n), n \in \mathbf{Z}$, is in its heart. This differs from (3.2.5) only by a shift. Then, the same way as for the motivic $t$-structure, the classical $t$-structure on $\operatorname{DTM}(X)$ is obtained by glueing the $t$-structures on the strata $X_{w}$. If necessary, we distinguish between these $t$-structures by using 'cl' (for example, $\tau \geqslant 0$,cl for the classical one), and ' $m$ ' for the motivic one.

The following lemma allows to lift the exactness properties of the six functors from the $\ell$-adic world to stratified Tate motives.

LEMMA 3.2.8. We assume that $S$ (in addition to the conditions in Notation 3.2.1) admits an $\ell$-adic realization functor $\rho_{\ell}$ (see Synopsis 2.1.1(xvii)). The restriction of $\rho_{\ell}$ to Tate motives,

$$
\rho_{\ell}: \operatorname{DTM}(X) \rightarrow \mathrm{D}_{\text {ét }}\left(X, \mathbf{Q}_{\ell}\right)
$$

is conservative and creates the motivic $t$-structure from the perverse $t$-structure on $\mathrm{D}_{\text {ét }}\left(X, \mathbf{Q}_{\ell}\right)$ (that is, $M \in \mathrm{DTM}(X) \leqslant 0$ is equivalent to $\rho_{\ell}(M) \in \mathrm{D}_{\text {ét }}\left(X, \mathbf{Q}_{\ell}\right) \leqslant 0$ and likewise for ' $\geqslant 0$ '). It also creates the classical $t$-structure on $\operatorname{DTM}(X)$ from the classical $t$-structure on the right hand category. In particular, for $M \in \mathrm{DTM}(X)$ the following properties $(a)-(b)$ are equivalent:

(a) $M \in \operatorname{MTM}(X)$ (respectively $M$ is in the heart of the classical $t$-structure),

(b) $\rho_{\ell}(M)$ is an $\ell$-adic perverse sheaf (respectively $\rho_{\ell}(M)$ is an $\ell$-adic 'honest' sheaf).

Proof. Since $\iota^{*}$ is conservative and creates the $t$-structure, we may replace $X$ by a connected component of $X^{+}$and assume $X$ is a cellular scheme. Now, the 
restriction to compact objects, $\left.\rho_{\ell}\right|_{\operatorname{DTM}(X)^{\mathrm{c}}}$ is exact (both for the motivic and the classical $t$-structure), which follows right from the definitions. This implies the exactness also on the Ind-completion of $\operatorname{DTM}(X)^{\mathrm{c}}$, which is $\operatorname{DTM}(X)$.

To check conservativity of $\rho_{\ell}$ we note that the $t$-structure on $\operatorname{DTM}(X)^{\mathrm{c}}$ is nondegenerate. This implies that the $t$-structure on its Ind-completion is again nondegenerate. By [BBD82, Proposition 1.3.7], the family of the cohomology functors $\mathrm{H}^{i}: \operatorname{DTM}(X) \rightarrow \operatorname{MTM}(X)(i \in \mathbf{Z})$ is therefore conservative, so it is enough to show the conservativity of $\left.\rho_{\ell}\right|_{\operatorname{MTM}(X)}$. Any mixed motive $M \in \operatorname{MTM}(X)$ is the filtered colimit of its compact subobjects $N \subset M$. Using the exactness of $\rho_{\ell}, \rho_{\ell}(N) \subset \rho_{\ell}(M)$, so the conservativity of $\rho_{\ell}$ on $\operatorname{MTM}(X)^{\mathrm{c}}$ implies the one on $\operatorname{MTM}(X)$. Using Lemma 3.2.4(ii), the conservativity of $\left.\rho_{\ell}\right|_{\operatorname{MTM}(X)^{\mathrm{c}}}$ can be shown as in [Wil08, Theorem 3.9] (which considers the special case $X=\operatorname{Spec} k$ ).

REMARK 3.2.9. Since no $\ell$ is invertible in $\mathbf{Z}$, Lemma 3.2.8 does not literally apply to $S=\operatorname{Spec} \mathbf{Z}$. However, Lemma 3.2.8 can be extended to such cases, too. More precisely, suppose that:

- $\quad S$ is as in Notation 2.0.1, and satisfies the Beilinson-Soulé vanishing conjecture;

- $\quad$ there are primes $\ell_{1} \neq \ell_{2}$ such that $S\left[\ell_{1}^{-1}\right], S\left[\ell_{2}^{-1}\right]$ satisfy the Beilinson-Soulé vanishing conjecture.

Then the functor

$$
\begin{aligned}
& \operatorname{DTM}(X) \longrightarrow \operatorname{DTM}\left(X\left[\ell_{1}^{-1}\right]\right) \times \operatorname{DTM}\left(X\left[\ell_{2}^{-1}\right]\right) \\
& \stackrel{\rho \ell_{2} \times \rho_{\ell_{1}}}{\longrightarrow} \mathrm{D}_{\text {ét }}\left(X\left[\ell_{1}^{-1}\right], \mathbf{Q}_{\ell_{1}}\right) \times \mathrm{D}_{\text {ét }}\left(X\left[\ell_{2}^{-1}\right], \mathbf{Q}_{\ell_{2}}\right)
\end{aligned}
$$

has the same properties as $\rho_{\ell}$ in the statement above: it is conservative, and creates the motivic $t$-structure from the perverse $t$-structures on the right hand categories. Note that the first functor is conservative by Zariski descent for DM and creates the motivic $t$-structure.

By a slight abuse of language, we still refer to the situation above by saying that ' $S$ admits an $\ell$-adic realization functor'.

COROLlary 3.2.10. Suppose $S$ admits an $\ell$-adic realization functor. Let $\pi: X \rightarrow Y$ be a Whitney-Tate map of cellular Whitney-Tate stratified indschemes. If $\pi$ is quasifinite, then $\pi^{*}$ and $\pi_{!}$(respectively $\pi^{!}$and $\pi_{*}$ ) are right $t$-exact (respectively left $t$-exact) for the motivic $t$-structure.

Proof. This follows from Remark 3.2.9 and the classical statement for $\ell$-adic sheaves [BBD82, Section 4.2.4]. 
The following statement is a refinement of the detection of Tate motives (Lemma 3.1.20).

Lemma 3.2.11. Suppose $\pi: X \rightarrow Y$ is a stratified map of cellular Whitney-Tate stratified ind-schemes such that $\tilde{\pi}_{\sharp} 1 \rightarrow 1$ has a section. If $\pi$ is smooth of constant relative dimension $d$, then $\pi^{*}[d]$ preserves and detects the motivic $t$-structure in the following sense:

$$
\begin{aligned}
& \pi^{*} M[d] \in \operatorname{DTM}(X)^{\geqslant 0} \Leftrightarrow M \in \operatorname{DTM}(Y)^{\geqslant 0} \\
& \pi^{*} M[d] \in \operatorname{DTM}(X)^{\leqslant 0} \Leftrightarrow M \in \operatorname{DTM}(Y)^{\leqslant 0} .
\end{aligned}
$$

Proof. Using smooth base change, we immediately reduce to $Y=Y^{+}$(and hence $\pi=\tilde{\pi})$, and further to the case where $Y$ is a cellular scheme. The implication ' $\Leftarrow$ ' then reduces to the observation that $\pi^{*}[d] 1_{Y}\left[d_{Y}\right]=1_{X}\left[d_{X}\right]$ lies in $\operatorname{MTM}(X)$ where $d_{Y}$ (respectively $d_{X}$ ) is the relative dimension of $Y$ (respectively $X$ ).

Conversely, assume $\pi^{*} M[d] \in \operatorname{DTM}(X)^{\leqslant 0}$ or equivalently, $\pi^{*} M[d]$ is left orthogonal to all objects in $\operatorname{DTM}(X)^{\geqslant 1}$. Since $\pi^{*}[d]$ is exact, this includes in particular all $\pi^{*}[d] M^{\prime}$ with $M^{\prime} \in \operatorname{DTM}(Y)^{\geqslant 1}$. Then, $\operatorname{Hom}\left(M, M^{\prime}\right) \rightarrow$ $\operatorname{Hom}\left(\pi^{*} M, \pi^{*} M^{\prime}\right)=\operatorname{Hom}\left(\pi_{\sharp} \pi^{*} M, M^{\prime}\right)$ is injective since $\pi_{\sharp} \pi^{*} M=\pi_{\sharp} 1 \otimes M$ contains $M$ as a direct summand by assumption. Therefore, $M$ is orthogonal to $M^{\prime}$, that is, $M \in \mathrm{DTM}(Y)^{\leqslant 0}$. An analogous argument works for $\geqslant 0$ which implies the lemma.

LEMMA 3.2.12. Let $\pi: X \rightarrow Y$ be a smooth surjective map of schemes of relative dimension $d$ with connected fibers. Let $Y$ be equipped with a cellular Whitney-Tate stratification, and equip $X$ with the preimage stratification (Example 3.1.17(i)). If $X$ is a cellular $Y$-scheme (Definition 3.1.5), then

$$
\pi^{!}[-d]=\pi^{*}[d](d): \operatorname{MTM}(Y) \rightarrow \operatorname{MTM}(X)
$$

is a fully faithful functor.

Proof. We may assume that $Y$ (and hence $X$ [Sta17, Tag 0378]) is connected. We proceed by induction on the number of cells in $X$. If $X$ is a single cell (over $Y$ ), then localization, homotopy invariance, and the Beilinson-Soule vanishing for $Y$ show the following isomorphism for $M, N \in \operatorname{MTM}(Y)$ :

$$
\operatorname{Hom}_{\mathrm{DM}(X)}\left(\pi^{*} M, \pi^{*} N\right)=\operatorname{Hom}_{\mathrm{DM}(Y)}(M, N) .
$$

For the inductive step consider a minimal cell $Z \stackrel{i}{\rightarrow} X \stackrel{j}{\leftarrow} U:=X \backslash Z$. Since $X$ is connected, and has at least two strata by assumption, the codimension 
$c:=\operatorname{codim}_{X} Z>0$ is positive. We use relative purity to compute the localization triangle: $i_{i} i^{!} \pi^{*} N=i_{*} i^{*} \pi^{*} N(-c)[-2 c] \rightarrow \pi^{*} N \rightarrow j_{*} j^{*} \pi^{*} N$. Let $\pi_{Z}:=\pi \circ i$, $\pi_{U}:=\pi \circ j$. Applying $\operatorname{Hom}_{X}\left(\pi^{*} M,-\right)$ gives a 4-term exact sequence

$$
\begin{aligned}
& \operatorname{Hom}_{Z}\left(\pi_{Z}^{*} M, \pi_{Z}^{*} N(-c)[-2 c]\right) \rightarrow \operatorname{Hom}_{X}\left(\pi^{*} M, \pi^{*} N\right) \rightarrow \operatorname{Hom}_{U}\left(\pi_{U}^{*} M, \pi_{U}^{*} N\right) \\
& \quad \rightarrow \operatorname{Hom}_{Z}\left(\pi_{Z}^{*} M, \pi_{Z}^{*} N(-c)[-2 c+1]\right) .
\end{aligned}
$$

The outer terms vanish by the Beilinson-Soulé condition for $Z$ since $-2 c+1<0$ which follows from the corresponding condition for $Y$ by Lemma 3.2.4.

\subsubsection{Equivariant mixed Tate motives.}

DEFINITION 3.2.14. Let $G$ be an ordinary presheaf of groups acting on $X$ over $S$. We define the category of $G$-equivariant mixed Tate motives as the homotopy pullback

$$
\operatorname{MTM}_{G}(X) \stackrel{\text { def }}{=} \operatorname{MTM}(X) \times_{\mathrm{DM}(X)} \operatorname{DM}(G \backslash X),
$$

or equivalently as the full subcategory of $\operatorname{DM}(G \backslash X)$ (Definition 2.2.6) of those objects whose underlying motive is a mixed Tate motive, with respect to the given stratification on $X$.

The preceding definition works even if the $G$-action is incompatible with the stratification. However, to prove that $\operatorname{MTM}_{G}(X)$ is abelian we need that the $G$-action respects the stratification:

Proposition 3.2.15. In the situation of Proposition 3.1.27, assume in addition that each $G_{i}$ is cellular. Then $\mathrm{DTM}_{G}(X)$ admits a t-structure such that the forgetful functor $\mathrm{DTM}_{G}(X) \rightarrow \operatorname{DTM}(X)$ is t-exact. Its heart identifies with the category $\mathrm{MTM}_{G}(X)$ defined above. It is a $\mathbf{Q}$-linear abelian category and can be computed as

$$
\operatorname{MTM}_{G}(X)=\underset{i}{\operatorname{colim}} \lim _{j \geqslant i} \operatorname{MTM}_{G_{j}}\left(X_{i}\right) .
$$

The colimit is taken in the bicategory of cocomplete categories and continuous functors (or in $\mathrm{DGCat}_{\text {cont }}$ ). Transition functors are the pushforwards along $X_{i} \rightarrow$ $X_{i^{\prime}}$. The limit is formed using the restriction functors along $G_{j} \rightarrow G_{j^{\prime}}$.

The category $\operatorname{MTM}_{G}(X)$ is compactly generated, that is, $\operatorname{MTM}_{G}(X)=$ $\operatorname{Ind}\left(\mathrm{MTM}_{G}(X)^{\mathrm{c}}\right)$ is the ind-completion of the subcategory of compact objects. The latter is given by a similar formula, namely

$$
\operatorname{MTM}_{G}(X)^{\mathrm{c}}=\underset{i}{\operatorname{colim}} \lim _{j \geqslant i} \operatorname{MTM}_{G_{j}}\left(X_{i}\right)^{\mathrm{c}},
$$

where now, however, the colimit is taken in the bicategory of categories with not necessarily continuous functors. 
REMARK 3.2.16. Colloquially speaking, a compact mixed $G$-equivariant Tate motive on $X$ is therefore simply a $G_{j}$-equivariant mixed Tate motive on some $X_{i}$, where $j \geqslant i$ is arbitrary.

Proof. By Proposition 3.1.27, we have

$$
\operatorname{DTM}_{G}(X)=\text { colim } \lim _{j \geqslant i} \lim \operatorname{DTM}^{!}\left(\operatorname{Bar}\left(G_{j}, X_{i}\right)\right) .
$$

As explained there, the outer colimit is using *-pushforward along the closed immersions $t_{i, i^{\prime}}: X_{i} \rightarrow X_{i^{\prime}}$. The middle limit uses the restriction functors (which are equivalences) and the functors in the limit are !-pullbacks (along the maps $f$ in $\operatorname{Bar}\left(G_{j}, X_{i}\right)$, which are smooth maps). The latter limit does not change up to equivalence if we replace these pullback functors $f^{!}$by $f^{!}[-d]$, where $d$ is the relative dimension of $f$ (which is finite, since $G_{j}$ is of finite type). Both these shifted transition functors and also the $\left(t_{i j}\right)_{*}$ are t-exact by Lemma 3.2.11 and Corollary 3.2.10, respectively. Using Lemma 3.2.18, we get a $t$-structure whose heart is as claimed above.

Lemma 3.2.18. Consider a diagram of stable $\infty$-categories $\mathcal{C}_{i}$ and exact functors $F_{i j}: \mathcal{C}_{i} \rightarrow \mathcal{C}_{j}$ between them. We suppose that all $\mathcal{C}_{i}$ are equipped with $t$-structures $\mathcal{C}_{i}^{\geqslant 0}$ (we use cohomological notation) and that the functors $F_{i j}$ are left t-exact, that is, preserve the ' $\geqslant 0$ '-subcategories. Let

$$
\mathcal{C}:=\lim _{F_{i j}} \mathcal{C}_{i}
$$

(i) The subcategory $\mathcal{C} \geqslant 0:=\lim _{F_{i j}} \mathcal{C}_{i}^{\geqslant 0}$ on $\mathcal{C}$ determines a $t$-structure. (Thus, $M \in$ $\mathcal{C} \geqslant 0$ if and only if all the projections $p_{i}(M) \in \mathcal{C}_{i}$ under the canonical maps $p_{i}: \mathcal{C} \rightarrow \mathcal{C}_{i}$ lie in $\mathcal{C}_{i}^{\geqslant 0}$.) If the $F_{i j}$ are in addition right $t$-exact, then the $p_{i}$ also create the ' $\leqslant 0$ ' part of the $t$-structure.

(ii) Suppose in addition that $I$ is filtered, that the $\mathcal{C}_{i}$ are presentable, and that the $F_{i j}$ have left adjoints $G_{i j}: \mathcal{C}_{j} \rightarrow \mathcal{C}_{i}$ which are t-exact and fully faithful. Using (i) and Lemma 2.3.2, we consider the induced t-structure on colim $_{G_{i j}} \mathcal{C}_{i} \stackrel{\cong}{\rightarrow}$. Here, colim denotes the colimit in the $\infty$-category of presentable $\infty$-categories with continuous functors. Then the canonical functors $\mathcal{C}_{i} \rightarrow \operatorname{colim}_{G_{i j}} \mathcal{C}_{i}$ are t-exact.

Proof. (i): The adjunctions $\left(\tau_{i}^{\geqslant 0}\right.$, incl $_{i}$ ) for the $\mathcal{C}_{i}$ 's propagate to one on $\mathcal{C}$, and the limit of the inclusion functors is fully faithful since the counit map of the adjunction for $\mathcal{C}$ is the limit of the counit maps for the $\mathcal{C}_{i}$, hence an equivalence, so that incl is indeed fully faithful. The subcategory $\mathcal{C} \geqslant 0 \subset \mathcal{C}^{0}$ is closed under 
extensions since the $p_{i}$ preserve finite (co)limits, in particular extensions. If the $F_{i j}$ are right exact, then the full subcategory $\mathcal{C}^{\leqslant-1}:=\lim _{F_{i j}} \mathcal{C}_{i}^{\leqslant-1} \subset \mathcal{C}$ agrees with $\operatorname{ker}\left(\tau^{\geqslant 0}=\lim \tau_{i}^{\geqslant 0}: \mathcal{C} \rightarrow \mathcal{C}^{\geqslant 0}\right)$.

(ii): By Lemma 2.3.2(ii) and the full faithfulness assumption, the composition ins $_{i}: \mathcal{C}_{i} \rightarrow \operatorname{colim}_{G_{i j}} \mathcal{C}_{i} \cong \lim _{F_{i j}} \mathcal{C}_{i}$ satisfies $p_{j}\left(\operatorname{ins}_{i}(M)\right)=F_{i j}(M)$ for $j<i$ and $G_{i j}(M)$ for $j \geqslant i$. If $M \in \mathcal{C}_{i}^{\geqslant 0}$, then both $F_{i j}(M)$ and $G_{i j}(M)$ are in $\mathcal{C}_{j}^{\geqslant 0}$ using the left exactness assumptions. Thus ins $s_{i}$ is left t-exact. To show the right t-exactness of ins ${ }_{i}$, we show that $\operatorname{Hom}_{\mathcal{C}}\left(\operatorname{ins}_{i} M, N\right)=\lim _{j} \operatorname{Hom}_{\mathcal{C}_{j}}\left(p_{j}\left(\operatorname{ins}_{i} M\right), p_{j}(N)\right)=0$ for any $M \in \mathcal{C}_{i}^{\leqslant-1}$ and $N \in \mathcal{C} \geqslant 0$. We may restrict the limit to $j>i$, in which case the $j$ th term reads $\operatorname{Hom}_{\mathcal{C}_{j}}\left(G_{i j} M, p_{j}(N)\right)$ which is contractible since $p_{j}(N) \in \mathcal{C}_{j}^{\geqslant 0}$ and $G_{i j}(M) \in \mathcal{C}_{j}^{\leqslant-1}$.

REMARK 3.2.19. To connect the category $\operatorname{MTM}_{G}(X)$ more closely to classical notions, we suppose for simplicity of notation that $G$ is algebraic and $X$ a scheme. The proof above shows that $\operatorname{MTM}_{G}(X)$ is equivalent to

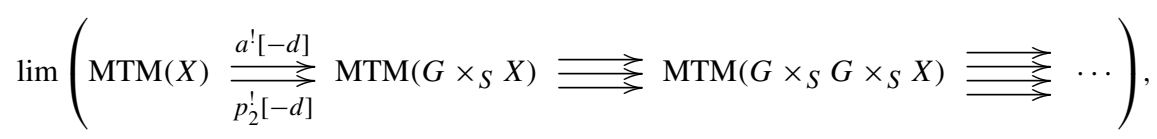

where $d=\operatorname{dim} G / S$. Using the t-exactness of $e^{!}[d], e: X \rightarrow G \times{ }_{S} X$, and the cofinality of the subcategory $\left(\Delta^{+}\right)^{\text {op }}$ of injective maps in $\Delta^{\text {op }}$, we may equivalently form the limit over the full cosimplicial diagram also involving the maps built using the unit sections. On the other hand, since MTM is an ordinary category, the limit does not change if we drop all terms $\operatorname{MTM}\left(G^{\times n} \times X\right)$ for $n \geqslant 3$. Thus, an object in $\operatorname{MTM}_{G}(X)$ is a datum

$$
\left(M_{0}, M_{1}, M_{2}, \varphi_{e}, \varphi_{a}, \varphi_{p_{2}}, \varphi_{\mathrm{id} \times a}, \varphi_{m \times \mathrm{id}}, \varphi_{p_{23}}\right)
$$

where $M_{0} \in \operatorname{MTM}(X), M_{i} \in \operatorname{DM}\left(G^{i} \times X\right)$ for $i=1,2$ and $\varphi_{a}: a^{!}[d] M_{0} \rightarrow M_{1}$, $\varphi_{\mathrm{id} \times a}:(\mathrm{id} \times a)^{!}[d] M_{1} \rightarrow M_{2}$, and so on are equivalences. Up to equivalence, we may further replace $a^{!}[d]$ by $a^{*}[-d]$, which description is equivalent to the standard definition of say equivariant perverse sheaves in [KW01, III.15, page 187].

The following proposition is a motivic variant of a well-known statement about equivariant perverse $\ell$-adic sheaves [KW01, III.15, page 188]. It allows us to easily construct equivariant mixed Tate objects, since we only have to check the existence of an isomorphism, as opposed to verifying higher coherences. 
Proposition 3.2.20. Let $G$ be a fiberwise connected smooth $S$-affine $S$-group whose underlying scheme is cellular. Let $X$ be a cellular Whitney-Tate stratified scheme with a stratified $G$-action. Then the forgetful functor

$$
\operatorname{MTM}_{G}(X) \rightarrow \operatorname{MTM}(X)
$$

is fully faithful and its image consists precisely of those motives $M \in \operatorname{MTM}(X)$ such that there is an isomorphism $a ! M[-\operatorname{dim} G] \cong p ! M[-\operatorname{dim} G]$ in $\operatorname{MTM}\left(G \times_{S}\right.$ $X$ ) where $a: G \times{ }_{S} X \rightarrow X$ (respectively $p: G \times{ }_{S} X \rightarrow X$ ) is the action (respectively projection). We call this condition the naive equivariance condition.

Proof. Fix $n$, and denote $H:=G^{\times s^{n}}$. Applying Lemma 3.2.12 to the projection map $p: H \times{ }_{S} X \rightarrow X$ we see that $p^{!}: \operatorname{MTM}(X) \rightarrow \operatorname{DM}\left(H \times{ }_{S} X\right)$ is fully faithful. This implies that !-pullback along the unit map $X \rightarrow H \times{ }_{S} X$ is fully faithful, hence our claim by Lemma 2.2.12.

COROLlary 3.2.21. In the situation of Proposition 3.2.20, suppose $G$ acts trivially on $X$. Then there is an equivalence $\operatorname{MTM}_{G}(X) \rightarrow \operatorname{MTM}(X)$. We therefore get

$$
\begin{aligned}
\mathrm{H}_{\mathrm{mot}}^{1}(B G, \mathbf{Q}) & \stackrel{\text { def }}{=} \operatorname{Hom}_{\mathrm{DM}(G \backslash S)}(1,1[1])=\operatorname{Ext}_{\mathrm{MTM}_{G}(X)}^{1}(1,1)=\operatorname{Ext}_{\mathrm{MTM}(S)}^{1}(1,1) \\
& =\left(K_{-1}(S) \otimes \mathbf{Q}\right)^{(0)}=0 .
\end{aligned}
$$

Proof. For a trivial action, the condition $a^{!} M \cong p^{!} M$ is vacuous. We are done using that $\mathrm{Ext}^{1}$ in the heart of an $t$-structure agrees with homomorphisms in the original triangulated category [DG05, (1.1.5)]. The identification with $K$-theory uses the regularity of $S$.

In the following proposition we do not need to assume that $G$ or $H$ is cellular since we just work with the definition of $\operatorname{MTM}_{G}(X)$ and only use the $t$-structure on nonequivariant motives.

Proposition 3.2.22. Suppose we are in the situation of Proposition 3.1.23: $H \subset G$ is an inclusion of ordinary $\tau$-sheaves of $S$-groups, $X:=(G / H)^{\tau}$ be the quotient of $\tau$-sheaves which we assume to be a smooth finite type $S$-scheme equipped with a cellular Whitney-Tate stratification such that the base point $S \rightarrow X$ factors over $X^{+}$. Let $d:=\operatorname{dim} X / S$, and denote by $e: S / H \rightarrow G \backslash X$ be the map induced from the base point. Then the equivalence in Proposition 3.1.23 restricts to an equivalence

$$
e^{!}[d]: \operatorname{MTM}_{G}(X) \stackrel{\simeq}{\longrightarrow} \operatorname{MTM}_{H}(S) .
$$


Proof. We construct the inverse of the equivalence. Let $\pi: X \rightarrow S$ be the structure map. The functor $\pi^{!}[-d]$, being $t$-exact and conservative, creates the motivic $t$-structure, that is, for $M \in \operatorname{DTM}(S)$ we have $\pi^{!}[-d] M \in \operatorname{MTM}(X)$ if and only if $M \in \operatorname{MTM}(S)$. We then conclude using Proposition 3.1.23.

We now obtain a convenient description of generators of certain equivariant categories of Tate motives.

Proposition 3.2.23. Let $H \subset G$ be an inclusion of smooth $S$-affine $S$-groups, and suppose that the étale sheaf quotient $X=(G / H)^{\text {ét }}$ is a scheme equipped with a cellular Whitney-Tate stratification such that the base point $S \rightarrow X$ factors over $X^{+}$. Further, assume that $H$ is fiberwise connected and that its underlying scheme is cellular. Then the shifted!-pullback along the map $S \rightarrow H \backslash S \rightarrow G / X$ induces an equivalence

$$
\operatorname{MTM}_{G}(X) \stackrel{\cong}{\rightarrow} \operatorname{MTM}(S) .
$$

In particular, $\operatorname{MTM}_{G}(X)$ (respectively $\operatorname{DTM}_{G}(X)$ ) is generated by means of coproducts and extensions (respectively by means of colimits and arbitrary shifts) by motives of the form $1_{X}(n)[d], n \in \mathbf{Z}, d:=\operatorname{dim} X / S$.

Proof. This follows from Proposition 3.2.22, Corollary 3.2.21 and the fact that $\operatorname{MTM}(S)$ is generated by the motives $1(n)$.

We obtain the following corollary in the case $X$ has several strata.

COROLlary 3.2.24. Suppose a smooth $S$-affine $S$-group $G$ acts on a finite type scheme $X$ over $S$. Suppose that it carries a cellular Whitney-Tate stratification $X^{+}=\bigsqcup_{w \in W} X_{w} \rightarrow X$ where each stratum has the form $X_{w}=\left(G / H_{w}\right)^{\text {ét }}$ and satisfies all conditions in Proposition 3.2 .23 (in particular $H_{w}$ is fiberwise connected). We write $\iota_{w}: G \backslash X_{w} \rightarrow G \backslash X$ for the map of prestacks (whose étale sheafifications are Artin stacks) induced by the strata inclusions. Then $\operatorname{DTM}_{G}(X)$ is generated, by means of colimits and shifts by the objects $\left(l_{w}\right) ! 1(n)$, where $\iota_{w}: X_{w} \rightarrow X$ is the inclusion and $n \in \mathbf{Z}$, and $\left(\iota_{w}\right)$ ! is the left adjoint of $\iota_{w}^{\text {. }}$.

Proof. Let $X_{0} \subset X$ be an open stratum, and its complement $X_{1}$ stratified by the remaining strata. By Lemma 2.2.9, we have the following adjoints (left adjoints are depicted above their right adjoints)

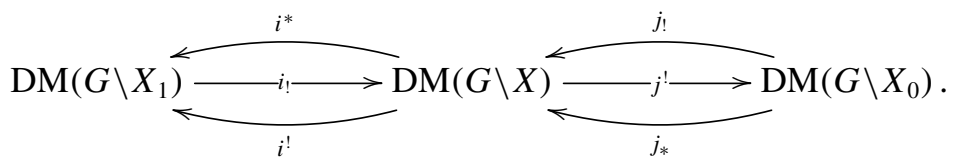


Moreover, $\left(i^{!}, j^{!}\right)$is conservative and $i^{*} i_{!}=\mathrm{id}, j^{!} j_{!}=\mathrm{id}$, so that the localization cofiber sequence in (2.1.5) carries over to the equivariant setting. By construction, the functors have their usual meaning if we forget the $G$-equivariance, so they preserve the subcategories $\operatorname{DTM}_{G}(-) \subset \mathrm{DM}(G \backslash-)$. Thus $\operatorname{DTM}_{G}(X)$ is generated by $j ! \mathrm{DTM}_{G}\left(X_{0}\right)$ and $i_{!} \mathrm{DTM}_{G}\left(X_{1}\right)$. This allows an induction on the number of strata, the case of a single stratum being Proposition 3.2.23.

3.3. Simple objects. In this section, $S$ is a scheme satisfying the conditions in Notation 2.0.1 which is moreover regular, satisfies the Beilinson-Soule vanishing conjecture as in (3.2.2) and admits an $\ell$-adic realization functor $\rho_{\ell}$ in the sense of Remark 3.2.9.

In order to describe the simple objects in the category $\operatorname{MTM}(X)$, we need to introduce the middle extension functor $j_{\text {!** }}$. This follows closely the classical theory [BBD82].

Let $\iota: X^{+}=\bigsqcup_{w \in W} X_{w} \rightarrow X$ be a cellular Whitney-Tate stratified $S$-scheme of finite type, and denote $\iota_{w}:=\left.\iota\right|_{X_{w}}$. Let $j: U \rightarrow X$ be an open immersion, and assume $U^{+}:=U \times_{X} X^{+}=\bigsqcup_{w \in W_{U}} X_{w}$ for some subset $W_{U} \subset W$. In particular, $U$ is Whitney-Tate, and $j$ is a Whitney-Tate map of cellular stratified Whitney-Tate schemes.

For an object $A \in \operatorname{MTM}(U)$, we have $j_{!} A \in \mathrm{DTM}^{\leqslant 0}(X)$ (respectively $j_{*} A \in$ $\mathrm{DTM}^{\geqslant 0}(X)$ ) by definition of the $t$-structures. Hence, the natural map $j_{!} A \rightarrow j_{*} A$ factors as $j_{!} A \rightarrow{ }^{\mathrm{m}} \mathrm{H}^{0} j_{!} A \rightarrow{ }^{\mathrm{m}} \mathrm{H}^{0} j_{*} A \rightarrow j_{*} A$ where ${ }^{\mathrm{m}} \mathrm{H}^{0}$ denotes the 0th truncation with respect to the motivic $t$-structure on $\operatorname{DTM}(X)$.

Definition 3.3.1. The middle extension of $A$ along $j$ is defined as the image

$$
j_{! *} A \stackrel{\text { def }}{=} \operatorname{im}\left({ }^{\mathrm{m}} \mathrm{H}^{0} j_{!} A \rightarrow{ }^{\mathrm{m}} \mathrm{H}^{0} j_{*} A\right) \in \operatorname{MTM}(X) .
$$

By Corollary 3.2.6, $j_{\text {!* }}$ preserves compact objects.

LEMMA 3.3.3. Under the $\ell$-adic realization (cf. Lemma 3.2.8), one has $\rho_{\ell}\left(j_{! *} A\right) \simeq j_{! *}\left(\rho_{\ell}(A)\right)$. In particular, the middle extension $j_{! *} A$ is the unique extension $B \in \mathrm{DTM}(X)$ of $A$ with the property

$$
\begin{aligned}
& { }^{\mathrm{cl}} \mathrm{H}^{i}\left(\iota_{w}^{*} \rho_{\ell}(B)\right)=0 \quad \text { for } i \geqslant-\operatorname{dim}\left(X_{w} / S\right) \\
& \text { and }{ }^{\mathrm{cl}} \mathrm{H}^{i}\left(\iota_{w}^{!} \rho_{\ell}(B)\right)=0 \text { for } i \leqslant-\operatorname{dim}\left(X_{w} / S\right) \text {, }
\end{aligned}
$$

where ${ }^{\mathrm{cl}} \mathrm{H}^{i}$ denotes the ith cohomology with respect to the classical $t$-structure on $\mathrm{D}_{\text {ét }}\left(X, \mathbf{Q}_{\ell}\right)$. 
Proof. This follows from the parallel $\ell$-adic statement [BBD82, Proposition 2.1.9] and Remark 3.2.9 because the functors ${ }^{\mathrm{m}} \mathrm{H}^{0}$, $j_{\text {! }}$ and $j_{*}$ commute with $\rho_{\ell}$, as does the formation of images and kernels in the abelian category $\operatorname{MTM}(X)$.

LEMMA 3.3.4. If the $S$-scheme $X$ is cellular (hence smooth) of relative dimension $d$, then one has $1_{X}[d]=j_{! *} 1_{U}[d] \in \operatorname{MTM}(X)$. In addition, if $X$ is irreducible, then $1_{X}[d]$ is a simple object in $\operatorname{MTM}(X)$.

Proof. Since $X$ (and all the strata $X_{w}$ ) are smooth, one has $\iota_{w}^{!} 1=$ $1\left(-\operatorname{codim}_{X} X_{w}\right)\left[-2 \operatorname{codim}_{X} X_{w}\right]$, so the first claim follows from Lemma 3.3.3. If $X$ is irreducible, then $1_{X}[d]$ is simple by adapting [BBD82, Lemma 4.3.3] to our set-up.

Let $\iota: X^{+}=\bigsqcup_{w \in W} X_{w} \rightarrow X$ be a stratified $S$-ind-scheme. As in (3.1.4), we write $X$ as the colimit of the stratified $S$-schemes ${\overline{X_{w}}}^{+}=\bigsqcup_{v \leqslant w} X_{v} \rightarrow \overline{X_{w}}$. The $\left.\operatorname{map} \iota\right|_{X_{w}}: X_{w} \rightarrow X$ factors as

$$
X_{w} \stackrel{j_{w}}{\longrightarrow} \overline{X_{w}} \stackrel{i_{w}}{\longrightarrow} X,
$$

where $j_{w}$ is a dense open immersion and $i_{w}$ a closed immersion. Note that if $X^{+} \rightarrow X$ is cellular Whitney-Tate stratified, then ${\overline{X_{w}}}^{+} \rightarrow \overline{X_{w}}$ is cellular WhitneyTate stratified.

DEFINITION 3.3.5. In the above situation, the intersection motive is defined for each $w \in W, n \in \mathbf{Z}$ as

$$
\mathrm{IC}_{w}(n) \stackrel{\text { def }}{=} i_{w, *}\left(j_{w, ! *} 1_{X_{w}}(n)\left[d_{w}\right]\right) \in \operatorname{MTM}(X)^{\mathrm{c}},
$$

where $d_{w}$ is the relative dimension of the cellular $S$-scheme $X_{w}$. (Corollary 3.2.10 and $i_{w, *}=i_{w, !}$ shows $\operatorname{IC}_{w}(n)$ is a mixed Tate motive.)

REMARK 3.3.7. We emphasize that the existence of intersection motives (without assuming any standard conjectures) in this special situation is guaranteed by the cellularity assumptions and the Beilinson-Soule conjecture. Another, somewhat orthogonal case where the intersection motive exists is the case of singular proper surfaces [Wil16].

THEOREM 3.3.8. Let $X^{+}=\bigsqcup_{w \in W} X_{w} \rightarrow X$ be a cellular Whitney-Tate stratified ind-scheme.

(i) The category of compact objects $\operatorname{MTM}(X)^{\mathrm{c}}$ is Artinian and Noetherian: every object is of finite length. 
(ii) If $X_{w}$ is irreducible for each $w \in W$, then the twisted intersection motives $\operatorname{IC}_{w}(n) \in \operatorname{MTM}(X)^{\mathrm{c}}$ are simple. In addition, if $X_{w}$ is a cell for each $w \in W$, then the simple objects in $\operatorname{MTM}(X)^{\mathrm{c}}$ are precisely the intersection motives $\mathrm{IC}_{w}(n)$ for $w \in W, n \in \mathbf{Z}$.

Proof. Again (i) is immediate from $\rho_{\ell}$ being conservative, cf. Remark 3.2.9. For (ii), we reduce to the case where $X$ is a cellular Whitney-Tate stratified $S$-scheme. The intersection motives $\operatorname{IC}_{w}(n)$ are simple because they map to simple objects under the $\ell$-adic realization. As in [BBD82, Section 4.3.4] one can proceed by Noetherian induction to see that every simple object is obtained in this way if each $X_{w}$ is a cell: Let $j: U \rightarrow X$ be an open stratum with closed complement $i: X \backslash U \rightarrow X$, and assume by induction that (ii) holds for objects in $i_{*} \operatorname{MTM}(X \backslash U)^{\mathrm{c}} \subset \operatorname{MTM}(X)^{\mathrm{c}}$. If $A \in \operatorname{MTM}(X)^{\mathrm{c}}$, then $j^{*} A \in \operatorname{MTM}(U)^{\mathrm{c}}$ is by construction a successive extension of twisted $1_{U}[d](n)$ with $d$ being the relative dimension of $U$ and $n \in \mathbf{Z}$ arbitrary (the category $\operatorname{MTM}(U)$ is a category of Tate type, then apply [Lev93, Theorem 1.4(iii)]). Hence, the simple constituents of $j_{! *} j^{*} A$ are of the desired form. The exact sequences in $\operatorname{MTM}(X)$,

$$
\begin{aligned}
& 0 \rightarrow i_{*}{ }^{\mathrm{m}} \mathrm{H}^{-1}\left(i^{*} A\right) \rightarrow{ }^{\mathrm{m}} \mathrm{H}^{0} j_{!} j^{*} A \rightarrow A \rightarrow i_{*}{ }^{\mathrm{m}} \mathrm{H}^{0}\left(i^{*} A\right) \rightarrow 0 \\
& 0 \rightarrow i_{*}{ }^{\mathrm{m}} \mathrm{H}^{0}\left(i^{!} A\right) \rightarrow A \rightarrow{ }^{\mathrm{m}} \mathrm{H}^{0} j_{*} j^{*} A \rightarrow i_{*}{ }^{\mathrm{m}} \mathrm{H}^{1}\left(i^{!} A\right) \rightarrow 0
\end{aligned}
$$

give that the cokernel of $j_{! *} j^{*} A \subset \operatorname{im}\left(A \rightarrow{ }^{\mathrm{m}} \mathrm{H}^{0} j_{*} j^{*} A\right)$ lies in the category $i_{*} \operatorname{MTM}(X \backslash U)^{\mathrm{c}}$. Part (ii) follows by induction.

\section{Loop groups and their flag varieties}

In this section, we study loop groups and their flag varieties associated with Chevalley groups $G$ over $\mathbf{Z}$. We then gather some results about the partial affine flag variety $\mathrm{Fl}=L G / \mathcal{P}$ associated with a parahoric subgroup $\mathcal{P} \subset L G$. A final goal is to show that $\mathrm{Fl}$ has the structure of a cellular stratified ind-scheme in the sense of Definition 3.1.1. Results over general base schemes $S$ are deduced in Section 4.4 by base change.

4.1. Group-theoretic notation. We fix a Chevalley group scheme $G$ over $\mathbf{Z}$, that is, a smooth affine $\mathbf{Z}$-group scheme whose geometric fibers are connected reductive groups, and which admits a maximal torus defined over $\mathbf{Z}$, cf. [Con14, Section 6.4]. We fix a maximal $\mathbf{Z}$-torus $T \subset G$ which is automatically split, cf. [Con14, Example 5.1.4] (because the Galois module $X^{*}\left(T_{\overline{\mathbf{Q}}}\right)$ is necessarily unramified, and hence trivial). Let $B \subset G$ be a Borel subgroup defined over $\mathbf{Z}$ 
and containing $T$. We obtain a Borel triple of smooth $\mathbf{Z}$-group schemes

$$
T \subset B \subset G \text {. }
$$

(i) Cocharacters. There is the natural pairing of finitely generated free $\mathbf{Z}$-modules $\langle-,-\rangle: X^{*}(T) \times X_{*}(T) \rightarrow \mathbf{Z}$ where $X^{*}(T):=\operatorname{Hom}\left(T, \mathbf{G}_{m, \mathbf{Z}}\right)$ (respectively $X_{*}(T):=\operatorname{Hom}\left(\mathbf{G}_{m, \mathbf{Z}}, T\right)$ ) is the group of characters (respectively of cocharacters) defined over $\mathbf{Z}$.

(ii) Roots. Let $R \subset X^{*}(T)$ be the roots associated with $(G, T)$, and let $R_{+}$the subset of positive roots defined by $B$.

(iii) Affine roots. Let $\mathscr{A}:=X_{*}(T) \otimes \mathbb{R}$. The roots $R$ are regarded as linear maps on $\mathscr{A}$. Adding integers to their values gives the set $\mathcal{R}:=R+\mathbf{Z}$ of affine roots which are then affine linear maps $\mathscr{A} \rightarrow \mathbb{R}$.

(iv) Standard apartment. The vector space $\mathscr{A}$ equipped with the simplicial structure defined by the hyperplanes $\operatorname{ker}(\alpha)$ for $\alpha \in \mathcal{R}$ is called the standard apartment. The connected components of $\mathscr{A} \backslash\left(\bigcup_{\alpha \in \mathcal{R}} \operatorname{ker}(\alpha)\right)$ are called alcoves. For any alcove $\mathbf{a}$, its closure $\overline{\mathbf{a}}$ is a disjoint union of facets $\mathbf{f}$ which are locally closed by convention and may have dimension ranging from 0 to $\operatorname{dim}_{\mathbb{R}}(\mathscr{A}$ ) (for example if $\overline{\mathbf{a}}$ is a triangle, then it is decomposed into three vertices, three edges and one alcove). Thus, $\mathscr{A}$ decomposes into a disjoint union of facets. There is a unique alcove $\mathbf{a}_{0}$ called the base alcove which lies in the chamber of $\mathscr{A}$ defined by $R_{+}$, and which contains 0 in its closure. A point in $\mathscr{A}$ is called special if every hyperplane $\operatorname{ker}(\alpha), \alpha \in \mathcal{R}$ is parallel to a hyperplane passing through that point. The base point $0 \in \mathscr{A}$ is always special. Note that if $\operatorname{ker}(\alpha)$ contains a point $x \in \mathscr{A}$, then it contains the unique facet $\mathbf{f}$ with $x \in \mathbf{f}$.

(v) Weyl groups. Let $W_{0}$ denote the Weyl group of the root system $R$ which acts on $\mathscr{A}$ by linear transformations. The Iwahori-Weyl group (or extended affine Weyl group) $W:=X_{*}(T) \rtimes W_{0}$ acts on $\mathscr{A}$ by affine linear transformations permuting transitively the set of alcoves in $\mathscr{A}$. For each $\alpha \in \mathcal{R}$, we have the reflection $s_{\alpha} \in W$ along the hyperplane $\operatorname{ker}(\alpha)$. The group $W$ acts on $\mathcal{R}$ via $w \alpha: \mathscr{A} \rightarrow \mathbb{R}$, $x \mapsto \alpha\left(w^{-1} x\right)$. We have the relation $w s_{\alpha} w^{-1}=s_{w \alpha}$ for all $\alpha \in \mathcal{R}$. For each facet $\mathbf{f} \subset \mathscr{A}$, we denote by $W_{\mathbf{f}} \subset W$ the subgroup generated by the reflections $s_{\alpha}$ such that $\mathbf{f} \subset \operatorname{ker}(\alpha)$, that is, $\left.\alpha\right|_{\mathbf{f}} \equiv 0$. We remark that $W_{\mathbf{f}}$ is finite.

(vi) Dominant cocharacters. The monoid of dominant cocharacters is

$$
X_{*}(T)_{+} \stackrel{\text { def }}{=}\left\{\lambda \in X_{*}(T) \mid\langle a, \lambda\rangle \geqslant 0 \forall a \in R_{+}\right\} .
$$

This monoid is equipped with the dominance partial order defined by: $\lambda \leqslant \mu$ if and only if $\mu-\lambda$ is a sum of positive coroots with coefficients in $\mathbf{Z}_{\geqslant 0}$. 
4.2. Parahoric subgroups. The loop group $L G$ is the group functor on the category of rings

$$
L G: R \longmapsto G(R((\varpi))),
$$

where $R((\varpi))$ denotes the ring of Laurent series in the formal variable $\varpi$. Since $G$ is affine and of finite type, the loop group $L G$ is representable by an ind-affine indscheme, cf. [PR08, Section 1(a)] (or [HR18b, Lemma 3.2] in greater generality). In particular, it is an fpqc sheaf on the category of rings, cf. Appendix A.1 below.

We are interested in certain pro-algebraic closed subgroups $\mathcal{P} \subset L G$, called parahoric subgroups. These subgroups should be regarded as infinite-dimensional analogues of parabolic subgroups in linear algebraic groups. We first give the guiding examples. The general notion defined in Lemma 4.2.4 is needed in the proof of Theorem 5.1.1. It requires some Bruhat-Tits theory [BT84].

EXAMPLE 4.2.2. (i) The positive loop group $L^{+} G$ is the group functor

$$
L^{+} G: R \longmapsto G(R \llbracket \varpi \rrbracket),
$$

where $R \llbracket \varpi \rrbracket \subset R((\varpi))$ is the subring of formal power series. Then as presheaves $L^{+} G=\lim _{i \geqslant 0} G_{i}$ with $G_{i}(R)=G\left(R[\varpi] /\left(\varpi^{i+1}\right)\right)$, and hence $L^{+} G$ is represented by a pro-algebraic $\mathbf{Z}$-group, see Appendix A.2 for conventions on pro-algebraic groups. The inclusion $L^{+} G \subset L G$ is relatively representable by a closed immersion, and makes $L^{+} G$ a closed $\mathbf{Z}$-subgroup functor of $L G$.

(ii) Example (i) is generalized as follows. For a standard parabolic subgroup $P \subset G$ (that is $P$ contains $B$ ), let $\mathcal{P} \subset L^{+} G$ (respectively $\mathcal{P}_{i} \subset G_{i}$ ) be the preimage of $P \subset G$ under the reduction map $L^{+} G \rightarrow G, \varpi \mapsto 0$ (respectively $G_{i} \rightarrow G_{0}=G$ ). Then $\mathcal{P}=\lim _{i \geqslant 0} \mathcal{P}_{i}$ is a pro-algebraic closed $\mathrm{Z}$-subgroup scheme of $L G$. If $P=B$, then the parahoric subgroup $\mathcal{B}:=\mathcal{P}$ is called the standard Iwahori subgroup.

Let $k$ be a field. Recall the classical notion of parahoric subgroups in $L G \otimes k$. Here and below $L G \otimes k$ is the restriction of $L G$ to the category of $k$-algebras; it can be computed as $L(G \otimes k)$. Let $\mathbf{f} \subset \mathscr{A}$ be a facet, and let $\mathcal{G}_{\mathbf{f}, k}$ be the associated parahoric $k \llbracket \varpi \rrbracket$-group scheme, that is, the neutral component of the unique algebraic $k \llbracket \varpi \rrbracket$-group scheme such that the generic fiber is $G \otimes k((\varpi))$, and such that the $k \llbracket \varpi \rrbracket$-points are the pointwise fixer of $\mathbf{f}$ in $G(k((\varpi)))$ (under its action on the Bruhat-Tits building). In particular, this defines for any $k$-algebra $R$ a subgroup $\mathcal{G}_{\mathbf{f}, k}(R \llbracket \varpi \rrbracket) \subset G(R((\varpi)))$. The parahoric $k$-subgroup $\mathcal{P}_{\mathbf{f}, k} \subset L G \otimes k$ associated with $\mathbf{f}$ is the group functor on the category of $k$-algebras defined by

$$
\mathcal{P}_{\mathbf{f}, k}: R \mapsto \mathcal{G}_{\mathbf{f}, k}(R \llbracket \varpi \rrbracket) .
$$


Again $\mathcal{P}_{\mathbf{f}, k}$ is a pro-algebraic $k$-group given by the inverse limit of the Weil restriction of scalars $\mathcal{P}_{\mathbf{f}, k, i}:=\operatorname{Res}_{k_{i} / k}\left(\mathcal{G}_{\mathbf{f}, k} \otimes_{k \llbracket \varpi \rrbracket} k_{i}\right)$ for $k_{i}=k[\varpi] /\left(\varpi^{i+1}\right)$. In particular, to any facet $\mathbf{f} \subset \mathscr{A}$ we have associated the family of pro-algebraic groups $\mathbf{f} \mapsto\left\{\mathcal{P}_{\mathbf{f}, k}\right\}_{k}$ where $k$ ranges over all fields. For the notion of strictly proalgebraic groups, we refer the reader to Section A.2.

LEMMA 4.2.4. For any facet $\mathbf{f} \subset \mathscr{A}$, there exists a unique flat closed subscheme $\mathcal{P}_{\mathbf{f}} \subset L G$ such that for every field $k$ one has $\mathcal{P}_{\mathbf{f}} \otimes k=\mathcal{P}_{\mathbf{f}, k}$ as subgroups of $L G \otimes k$. The group scheme $\mathcal{P}_{\mathrm{f}}$ is a strictly pro-algebraic $\mathbf{Z}$-group scheme with connected fibers. It is called the parahoric subgroup of $L G$ associated with $\mathbf{f}$.

Proof. By [PZ13, Section 4.2.2] applied with $\mathbf{Z} \llbracket \varpi \rrbracket$ as a base ring (cf. also [HR18a, Lemma 2.1]), there exists an algebraic $\mathbf{Z} \llbracket \varpi \rrbracket$-group scheme $\mathcal{G}_{\mathbf{f}}$ with connected fibers such that $\mathcal{G}_{\mathbf{f}} \otimes \mathbf{Z}((\varpi))=G \otimes \mathbf{Z}((\varpi))$, and $\mathcal{G}_{\mathbf{f}} \otimes k \llbracket \varpi \rrbracket=\mathcal{G}_{\mathbf{f}, k}$ for any field $k$. We define $\mathcal{P}_{\mathbf{f}}$ as the functor on the category of rings given by $R \mapsto \mathcal{G}_{\mathbf{f}}(R \llbracket \varpi \rrbracket)$. Then $\mathcal{P}_{\mathbf{f}}=\lim _{i \geqslant 0} \mathcal{P}_{\mathbf{f}, i}$ is a pro-algebraic Z-group with connected fibers where

$$
\mathcal{P}_{\mathbf{f}, i} \stackrel{\text { def }}{=} \operatorname{Res}_{\mathbf{Z}_{i} / \mathbf{Z}}\left(\mathcal{G}_{\mathbf{f}} \otimes_{\mathbf{Z} \llbracket \varpi} \mathbf{Z}_{i}\right)
$$

for $\mathbf{Z}_{i}=\mathbf{Z}[\varpi] /\left(\varpi^{i+1}\right)$. Note that each $\mathcal{P}_{\mathbf{f}, i}$ is an algebraic $\mathbf{Z}$-group with connected fibers, cf. the proof of [Ric16a, Lemma 2.11(ii)], and hence $\mathcal{P}_{\mathbf{f}}$ has connected fibers as well, cf. Lemma A.1(i).

In particular, $\mathcal{P}_{\mathbf{f}} \subset L G$ is a flat closed subscheme such that $\mathcal{P}_{\mathbf{f}} \otimes k=\mathcal{P}_{\mathbf{f}, k}$ for any field $k$. This shows existence. Being a flat closed subscheme, $\mathcal{P}_{\mathbf{f}}$ agrees with the flat closure (=scheme-theoretic image) of $\mathcal{P}_{\mathbf{f}, \mathbf{Q}} \subset L G \otimes \mathbf{Q}$ inside $L G$. This shows uniqueness, and the lemma follows.

REMARK 4.2.6. More generally, for every subset $\Omega \subset \mathscr{A}$ whose projection onto the semisimple part $\mathscr{A}_{\mathrm{ss}}$ is bounded (cf. [Tit79, Section 3.4.1]), there exists a smooth affine $\mathbf{Z} \llbracket \varpi \rrbracket$-group scheme $\mathcal{G}_{\Omega}$ with connected fibers by [PZ13, Section 4.2.2], respectively [HR18a, Lemma 2.1]. The associated $\mathbf{Z}$-subgroup schemes $\mathcal{P}_{\Omega} \subset L G$ are strictly pro-algebraic with connected fibers, and satisfy the favorable property $\mathcal{P}_{\Omega} \cap \mathcal{P}_{\Omega^{\prime}}=\mathcal{P}_{\Omega \cup \Omega^{\prime}}$, cf. the proof of Lemma 4.3.7(i) below.

The general notion of parahoric groups relates to Example 4.2.2 as follows. We have $\mathcal{P}_{0}=L^{+} G$ for the base point $0 \in \mathscr{A}$. The group $\mathcal{P}_{\mathbf{a}_{0}}=\mathcal{B}$ is the standard Iwahori, and the parahoric subgroups from Example 4.2.2(ii) correspond to the finitely many facets $\mathbf{f} \subset \mathscr{A}$ whose closure contains 0 , and which are itself contained in the closure of $\mathbf{a}_{0}$. More generally, we have $\mathcal{P}_{\mathbf{f}} \subset \mathcal{P}_{\mathbf{f}^{\prime}}$ if and only if $\mathbf{f}^{\prime}$ is 
contained in the closure of $\mathbf{f}$. The following lemma relates to Proposition 2.2.11, and we record it for later use.

LeMma 4.2.7. Let $\mathbf{f} \subset \mathscr{A}$ be a facet. For each $i \geqslant 0$, the kernel $\operatorname{ker}\left(\mathcal{P}_{\mathbf{f}, i+1} \rightarrow\right.$ $\left.\mathcal{P}_{\mathbf{f}, i}\right)$ is a vector group of dimension $\operatorname{dim}(G / \mathbf{Z})$. In particular, $\operatorname{ker}\left(\mathcal{P}_{\mathbf{f}} \rightarrow \mathcal{P}_{\mathbf{f}, 0}\right)$ is split pro-unipotent in the sense of Definition A.5, and each $\mathcal{P}_{\mathbf{f}, i}$ is a cellular $\mathbf{Z}$-scheme in the sense of Definition 3.1.5.

Proof. By Proposition A.9 (cf. also Example A.12(iii(a))), each kernel $U_{i}:=$ $\operatorname{ker}\left(\mathcal{P}_{\mathbf{f}, i+1} \rightarrow \mathcal{P}_{\mathbf{f}, i}\right)$ is a vector group of dimension $d:=\operatorname{dim}(G / \mathbf{Z})$, and thus $\operatorname{ker}\left(\mathcal{P}_{\mathbf{f}} \rightarrow \mathcal{P}_{\mathbf{f}, 0}\right)$ is split pro-unipotent.

In order to show that each $\mathcal{P}_{\mathbf{f}, i}$ is cellular, we consider the projection $\mathcal{P}_{\mathbf{f}, i} \rightarrow \mathcal{P}_{\mathbf{f}, 0}$. This is isomorphic to a relative affine space: by induction on $i$ it is enough to show that the $U_{i}$-torsor $\mathcal{P}_{\mathbf{f}, i+1} \rightarrow \mathcal{P}_{\mathbf{f}, i}$ is trivial which holds by Proposition A.6. Hence, the map is on the underlying schemes isomorphic to $\mathcal{P}_{\mathbf{f}, i} \times_{\mathbf{Z}} U_{i} \rightarrow \mathcal{P}_{\mathbf{f}, i}$. As every vector bundle on $\operatorname{Spec} \mathbf{Z}$ can be trivialized, we get $U_{i} \simeq \mathbf{A}_{\mathbf{Z}}^{d}$. Hence, it is enough to show that $\mathcal{P}_{\mathbf{f}, 0}=\mathcal{G}_{\mathbf{f}} \otimes_{\mathbf{Z} \llbracket \varpi \rrbracket} \mathbf{Z}$ is cellular. By construction [PZ13, Section 4.2.2(a)], the special fiber $\mathcal{G}_{\mathbf{f}} \otimes_{\mathbf{Z} \llbracket \varpi \rrbracket} \mathbf{Z}$ is also given by base changing the schematic root data defining $\mathcal{G}_{\mathbf{f}}$, and thus admits a semidirect product (Levi) decomposition into a split unipotent $\mathbf{Z}$-group scheme and a split reductive $\mathbf{Z}$-group scheme. As every split reductive $\mathbf{Z}$-group scheme is cellular by the Bruhat decomposition (for example [Jan03, Section 13] over $\mathbf{Z}$ ), the lemma follows.

REMARK 4.2.8. Lemma 4.2.7 holds with the same proof for the more general subgroups $\mathcal{P}_{\Omega} \subset L G$ from Remark 4.2.6. This is needed in Lemma 4.3.7 below in order to control the stabilizers, for example, of the $L^{+} G$-action on the affine Grassmannian.

For two facets $\mathbf{f}, \mathbf{f}^{\prime} \subset \mathscr{A}$ and for any field $k$, the combinatorics of the double coset $\mathcal{P}_{\mathbf{f}^{\prime}}(k) \backslash L G(k) / \mathcal{P}_{\mathbf{f}}(k)$ are determined by the double classes $W_{\mathbf{f}^{\prime}} \backslash W / W_{\mathbf{f}}$ in the Iwahori-Weyl group as follows, cf. Section 4.1(v) for notation. We have an identification as abstract groups

$$
W=\operatorname{Norm}_{G}(T)(\mathbf{Z}((\varpi))) / T(\mathbf{Z} \llbracket \varpi \rrbracket) .
$$

This identification is compatible with the decomposition $W=X_{*}(T) \rtimes W_{0}$, and works as follows. We have $X_{*}(T)=T(\mathbf{Z}((\varpi))) / T(\mathbf{Z} \llbracket \varpi \rrbracket), \lambda \mapsto \varpi^{-\lambda}$ as subgroups of (4.2.9) where we refer to [dCHL18, Section 3.2] for a discussion of the sign in the identification. Further, as $(G, T)$ is split, the Weyl group scheme $\operatorname{Norm}_{G}(T) / T=\underline{W}_{0}$ is constant by [Con14, Proposition 5.1.6]. Thus, its $\mathbf{Z} \llbracket \varpi \rrbracket$-valued (respectively $\mathbf{Z}((\varpi))$-valued) points identify with $W_{0}$ as a subgroup 
(respectively quotient) of (4.2.9) which gives the above identification compatible with the semidirect product structure.

Further, the subgroup $W_{\mathbf{f}} \subset W$ associated with a facet $\mathbf{f} \subset \mathscr{A}$ is identified with

$$
W_{\mathbf{f}}=\left(\operatorname{Norm}_{G}(T)(\mathbf{Z}((\varpi))) \cap \mathcal{P}_{\mathbf{f}}(\mathbf{Z})\right) / T(\mathbf{Z} \llbracket \varpi \rrbracket) .
$$

For example, if $\mathbf{f}$ is an alcove, then $W_{\mathbf{f}}=\{*\}$ is trivial. On the other extreme, if $\mathbf{f}=0$ is the base point (or any other special point), then $W_{0}$ is the finite Weyl group.

Lemma 4.2.11. Let $\mathbf{f}, \mathbf{f}^{\prime} \subset \mathscr{A}$ be facets. For any field $k$, there is a bijection of sets

$$
W_{\mathbf{f}^{\prime}} \backslash W / W_{\mathbf{f}} \rightarrow \mathcal{P}_{\mathbf{f}^{\prime}}(k) \backslash L G(k) / \mathcal{P}_{\mathbf{f}}(k), \quad W_{\mathbf{f}^{\prime}} w W_{\mathbf{f}} \mapsto \mathcal{P}_{\mathbf{f}^{\prime}}(k) \dot{w} \mathcal{P}_{\mathbf{f}}(k),
$$

where $\dot{w} \in L G(k)$ denotes the image of a representative of $w \in W$ under the map $L G(\mathbf{Z}) \rightarrow L G(k)$.

Proof. Since $T$ is split, the natural map $X_{*}(T)=\operatorname{Hom}\left(\mathbf{G}_{m, \mathbf{Z}}, T\right) \rightarrow \operatorname{Hom}\left(\mathbf{G}_{m, k}\right.$, $T \otimes k)=: X_{*}(T \otimes k)$ is an isomorphism for any field $k$. The discussion above implies that the natural map

$$
W \rightarrow\left(\operatorname{Norm}_{G}(T)(k((\varpi))) \cap \mathcal{P}_{\mathbf{f}}(k)\right) / T(k \llbracket \varpi \rrbracket)=: W_{k}
$$

is an isomorphism. For each facet $\mathbf{f}$, we also have the subgroup $W_{\mathbf{f}, k} \subset W_{k}$ which under the isomorphism $W=W_{k}$ is identified with $W_{\mathbf{f}}$. Thus, we obtain $W_{\mathbf{f}^{\prime}} \backslash W / W_{\mathbf{f}}=W_{\mathbf{f}^{\prime}, k} \backslash W_{k} / W_{\mathbf{f}, k}$. Now the lemma follows from the work of BruhatTits, cf. [PR08, Appendix, Proposition 8] (or [Ric16b, Theorem 1.4]).

The following important special cases of these double cosets relate to the partial affine flag varieties introduced in Definition 4.3.1 below.

EXAMPLE 4.2.12. (i) Let $\mathbf{f}^{\prime}=\mathbf{a}_{0}$, that is, $\mathcal{P}_{\mathbf{f}^{\prime}}=\mathcal{B}$ is the standard Iwahori subgroup. The $\mathcal{B}$-orbits on $\mathrm{Fl}_{\mathbf{f}}$ are parametrized by the infinite set $W / W_{\mathbf{f}}$. In particular, if $\mathbf{f}=0$ is the base point, that is, $\mathrm{Fl}_{0}=\mathrm{Gr}_{G}$ is the affine Grassmannian, then $W / W_{0}=X_{*}(T)$ is the group of cocharacters.

(ii) Let $\mathbf{f}^{\prime}=\mathbf{f}=0$, that is, $\mathcal{P}_{\mathbf{f}^{\prime}}=\mathcal{P}_{\mathbf{f}}=L^{+} G$. The $L^{+} G$-orbit on $\mathrm{Gr}_{G}$ are parametrized by the set $W_{0} \backslash W / W_{0}=W_{0} \backslash X_{*}(T)=X_{*}(T)_{+}$of dominant cocharacters defined in (4.1.2).

Let us recall some more structure of the group $W$. The choice of the base alcove $\mathbf{a}_{0}$ equips $W$ with the structure of a quasi-Coxeter group with length function 
$l: W \rightarrow \mathbf{Z}_{\geqslant 0}$ and Bruhat-Chevalley partial order ' $\leqslant$ ' as follows. The group $W$ acts on the apartment $\mathscr{A}$ by affine linear transformations permuting transitively all alcoves. According to the choice of base alcove $\mathbf{a}_{0}$, we have the finite set of simple affine reflections $\mathbb{S} \subset W$ which are given by the reflections along the walls of $\mathbf{a}_{0}$. The subgroup $W_{\text {aff }} \subset W$ generated by $\mathbb{S}$ is the affine Weyl group in the sense of [Bou68, VI, Section 2.1] associated with the based root system $R$. In particular, the pair $\left(W_{\text {aff }}, \mathbb{S}\right)$ is a Coxeter group. If $\operatorname{Stab}_{\mathbf{a}_{0}}=\left\{w \in W \mid w \cdot \mathbf{a}_{0}=\mathbf{a}_{0}\right\}$ viewed as a subgroup of $W$, then there is a semidirect product decomposition

$$
W=W_{\text {aff }} \rtimes \operatorname{Stab}_{\mathbf{a}_{0}} .
$$

Hence, every element $w \in W$ admits a decomposition

$$
w=s_{1} \cdot \ldots \cdot s_{q} \cdot \tau_{w},
$$

for some $s_{1}, \ldots, s_{q} \in \mathbb{S}$ and unique $\tau_{w} \in \operatorname{Stab}_{\mathbf{a}_{0}}$. The decomposition (4.2.14) is called reduced whenever the number $q \in \mathbf{Z}_{\geqslant 0}$ is minimal among all decompositions of $w$. Reduced decompositions are not unique, but $q$ only depends on $w$, and not on the choice of reduced decomposition.

The length of $w \in W$ is defined to be the unique number $l(w):=q \in \mathbf{Z}_{\geqslant 0}$ in some reduced decomposition (4.2.14). The partial order on $W$ is defined by the requirement $v \leqslant w$ if and only if $\tau_{v}=\tau_{w}$, and $v$ arises by deleting some of the $s_{i}$ in a reduced decomposition of $w$.

Let $\mathbf{f}, \mathbf{f}^{\prime} \subset \mathscr{A}$ be facets contained in the closure of $\mathbf{a}_{0}$. Then the quasi-Coxeter structure of $W$ induces on the double classes

$$
W_{\mathbf{f}^{\prime}} \backslash W / W_{\mathbf{f}}
$$

a length function $l=l\left(\mathbf{f}^{\prime}, \mathbf{f}\right)$ and a partial order $\leqslant={ }^{\mathbf{f}^{\prime}} \leqslant$ f , cf. [Ric13, Lemma $\left.1.6 \mathrm{ff}\right]$.

EXAMPLE 4.2.16. If $\mathbf{f}^{\prime}=\mathbf{f}=0$, then $W_{0} \backslash W / W_{0}=X_{*}(T)_{+}$, cf. Example 4.2.12(ii). By for example [Ric13, Corollary 1.8], the length function is computed as

$$
l: X_{*}(T)_{+} \rightarrow \mathbf{Z}_{\geqslant 0}, \quad \mu \mapsto\langle 2 \rho, \mu\rangle,
$$

where $2 \rho:=\sum_{a \in R_{+}} a \in X^{*}(T)$ is the sum of the positive roots. The partial order on $X_{*}(T)_{+}$specializes to the dominance order described in (4.1.2). Similarly, if $\mathbf{f}^{\prime}=\mathbf{a}_{0}$, and $\mathbf{f}=0$, then $W / W_{0}=X_{*}(T)$, and the length function is computed as

$$
l: X_{*}(T) \rightarrow \mathbf{Z}_{\geqslant 0}, \quad \mu \mapsto\left\langle 2 \rho, \mu^{\mathrm{dom}}\right\rangle-\#\left\{a \in R^{+} \mid\langle a, \mu\rangle<0\right\},
$$

where $\mu^{\text {dom }}$ is the unique dominant representative in $W_{0} \cdot \mu$. 
4.3. Stratifications on affine flag varieties. Fix two facets $\mathbf{f}, \mathbf{f}^{\prime} \subset \mathscr{A}$, and denote by $\mathcal{P}:=\mathcal{P}_{\mathbf{f}}, \mathcal{P}^{\prime}:=\mathcal{P}_{\mathbf{f}^{\prime}}$ the associated parahoric subgroups.

DEFINITION 4.3.1. The (partial) affine flag variety $\mathrm{Fl}=\mathrm{Fl}_{\mathbf{f}}$ associated with $\mathbf{f}$ is the étale sheaf quotient

$$
\mathrm{Fl} \stackrel{\text { def }}{=}(L G / \mathcal{P})^{\text {ét }} .
$$

Let us note that the quotient map $L G \rightarrow \mathrm{Fl}$ admits sections Zariski locally which follows from [Fal03, Definition $5 \mathrm{ff}$.]. Thus $\mathrm{Fl}(R)=L G(R) / \mathcal{P}(R)$ for any local ring $R$, and $\mathrm{Fl}$ agrees with the Zariski sheafification of the functor $R \longmapsto$ $L G(R) / \mathcal{P}(R)$. Here we use that the group $G$ is split.

By [HR18a, Lemma $2.1 \mathrm{ff}$, the sheaf $\mathrm{Fl}$ is representable by an ind-projective ind-scheme over $\mathbf{Z}$. If $\mathbf{f}=\mathbf{a}_{0}$ is the base alcove, then $\mathcal{P}=\mathcal{B}$ is the standard Iwahori subgroup, and $\mathrm{Fl}$ is the full affine flag variety. If $\mathbf{f}=0$ is the base point, then $\mathcal{P}_{0}=L^{+} G$, and $\mathrm{Fl}=\mathrm{Gr}_{G}$ is the affine Grassmannian. Further, the affine flag variety is equipped with a transitive left action of the loop group

$$
L G \times \mathrm{Fl} \rightarrow \mathrm{Fl}, \quad(g, x) \mapsto g \cdot x
$$

The restriction of the $L G$-action to the parahoric subgroup $\mathcal{P}^{\prime}$ is well-behaved in the following sense.

LEMMA 4.3.3. There exists a $\mathcal{P}^{\prime}$-stable presentation $\mathrm{Fl}=\operatorname{colim}_{i} \mathrm{Fl}_{i}$ where $\mathrm{Fl}_{i}$ are projective $\mathbf{Z}$-schemes, and the $\mathcal{P}^{\prime}$-action on each $\mathrm{Fl}_{i}$ factors through some $\mathcal{P}_{j}^{\prime}$ for $j \gg 0$.

Proof. The lemma immediately follows from Lemma A.5 because Fl is indprojective over $\mathbf{Z}$, and in particular of ind-finite type.

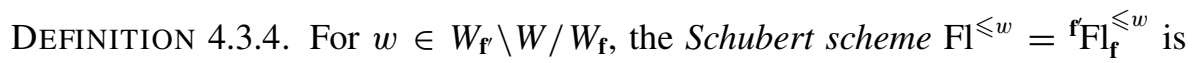
the scheme-theoretic image of the map

$$
\mathcal{P}^{\prime} \rightarrow \mathrm{Fl}, \quad p^{\prime} \mapsto p^{\prime} \cdot \dot{w} \cdot e,
$$

where $e \in \operatorname{Fl}(\mathbf{Z})$ is the base point, and $\dot{w} \in L G(\mathbf{Z})$ is a representative of $w$.

Let us justify the definition. It is clear from (4.2.10) that $\mathrm{Fl}^{\leqslant w}$ is independent of the choice of $\dot{w}$. Write $\mathrm{Fl}=\operatorname{colim}_{i} \mathrm{Fl}_{i}$ as in Lemma 4.3.3. Then there exists an $i \gg 0$ with $\dot{w} \in \mathrm{Fl}_{i}(\mathbf{Z})$, and hence the map (4.3.5) factors through $\mathrm{Fl}_{i}$ defining a map $\mathcal{P}^{\prime} \rightarrow \mathrm{Fl}_{i}$ of quasicompact separated schemes (hence a quasicompact map). 
By [Sta17, Tag 01R8], its scheme-theoretic image $\mathrm{Fl}^{\leqslant w}$ is the closed subscheme of $\mathrm{Fl}$ defined by the quasicoherent ideal sheaf $\operatorname{ker}\left(\mathcal{O}_{\mathrm{Fl}_{i}} \rightarrow \mathcal{O}_{\mathcal{P}^{\prime}}\right)$, and in particular, a projective $\mathbf{Z}$-scheme.

Usually, Schubert schemes (respectively Schubert varieties) in partial affine flag varieties are defined over fields as reduced orbit closures [PR08, Definition 8.3]. In the case of fields, the definition of Schubert varieties as reduced orbit closures agrees with the definition via scheme-theoretic images as in Definition 4.3.4. However, it is the latter notion which behaves well over more general base schemes, cf. Section 4.4 below. Here is the relation between Schubert varieties over fields with Schubert schemes over the integers.

LEMMA 4.3.6. For any field $k$, the underlying reduced locus of the base change $\mathrm{Fl}^{\leqslant w} \otimes_{\mathrm{Z}} k$ is the Schubert variety over $k$ associated with the element $w \in$ $W_{\mathbf{f}^{\prime}} \backslash W / W_{\mathbf{f}}$.

Proof. By Lemma 4.2.11, we obtain for each class $w$ a unique Schubert variety $\mathrm{Fl}_{k}^{\leqslant w}$ over $k$. We claim that the natural closed immersion $\mathrm{Fl}_{k}^{\leqslant w} \subset \mathrm{Fl}^{\leqslant w} \otimes_{\mathrm{z}} k$ is an isomorphism on reduced loci. We need to show that this map is an equality on the underlying topological spaces. For this fix a reduced expression of $w$ as in (4.2.14), and consider the Demazure resolution $D(w) \rightarrow \mathrm{Fl}^{\leqslant w}$ over the integers [Fal03, Definition $5 \mathrm{ff}$.]. Since $D(w) \rightarrow \mathrm{Fl}^{\leqslant w}$ is surjective, the base change $D(w) \otimes_{\mathbf{Z}} k \rightarrow \mathrm{Fl}^{\leqslant w} \otimes_{\mathbf{Z}} k$ is surjective as well [Sta17, Tag 01S1]. Since $D(w) \rightarrow \operatorname{Spec}(\mathbf{Z})$ is smooth, its formation commutes with base change so that $D(w) \otimes_{\mathbf{Z}} k \rightarrow \mathrm{Fl}_{k}^{\leqslant w}$ is the Demazure resolution over $k$. Thus, $\mathrm{Fl}_{k}^{\leqslant w} \subset \mathrm{Fl}^{\leqslant w} \otimes_{\mathbf{Z}} k$ is surjective, that is, an equality on the underlying topological spaces.

The following lemma is basic for the study of $\mathcal{P}^{\prime}$-orbits in $\mathrm{Fl}^{\leqslant w}$.

LEMMA 4.3.7. Let $w \in W_{\mathbf{f}^{\prime}} \backslash W / W_{\mathbf{f}}$.

(i) The stabilizer of $\mathcal{P}^{\prime}$ in $\dot{w} \cdot e \in \mathrm{Fl}(\mathbf{Z})$ is representable by a closed $\mathbf{Z}$-subgroup $\mathcal{P}_{w}^{\prime} \subset \mathcal{P}^{\prime}$. It is strictly pro-algebraic $\mathcal{P}_{w}^{\prime}=\lim _{i} \mathcal{P}_{w, i}^{\prime}$, and the scheme underlying each $\mathcal{P}_{w, i}^{\prime}$ is fiberwise connected and cellular.

(ii) The étale sheaf-theoretic image

$$
\mathrm{Fl}^{w}:=\mathcal{P}^{\prime} \cdot \dot{w} \cdot e \subset \mathrm{Fl}^{\leqslant w}
$$

is representable by an open subscheme which is smooth, fiberwise geometrically connected and dense over $\mathbf{Z}$. It agrees with the étale quotients $\mathrm{Fl}^{w}=\left(\mathcal{P}^{\prime} / \mathcal{P}_{w}^{\prime}\right)^{\text {ét }}=\left(\mathcal{P}_{i}^{\prime} / \overline{\mathcal{P}}_{w, i}^{\prime}\right)^{\text {ét }}$ for $i \gg 0$ where $\overline{\mathcal{P}}_{w, i}^{\prime} \subset \mathcal{P}_{i}^{\prime}$ is a fiberwise connected smooth cellular closed $\mathbf{Z}$-subgroup scheme. 
(iii) For each $v \leqslant w$, there is a quasicompact immersion $\mathrm{Fl}^{v} \rightarrow \mathrm{Fl}^{\leqslant w}$, and one has as sets

$$
\mathrm{Fl}^{\leqslant w}=\bigsqcup_{v \leqslant w} \mathrm{Fl}^{v},
$$

where ' $\leqslant$ ' denotes the partial order on $W_{\mathbf{f}^{\prime}} \backslash W / W_{\mathbf{f}}$.

Proof. For (i), the stabilizer is given by the closed $\mathbf{Z}$-subgroup $\mathcal{P}_{\mathbf{f}^{\prime}} \cap \mathcal{P}_{w \mathbf{f}} \subset L G$ where we used that $\mathcal{P}_{w \mathbf{f}}=\dot{w} \mathcal{P}_{\mathbf{f}} \dot{w}^{-1}$. As in Remark 4.2.6, we have for any subset $\Omega \subset \mathscr{A}$ whose projection onto the semisimple part $\mathscr{A}_{\mathrm{ss}}$ is bounded, an algebraic $\mathbf{Z} \llbracket \varpi \rrbracket$-group scheme $\mathcal{G}_{\Omega}$ with connected fibers. We apply this to $\Omega=\mathbf{f}^{\prime} \cup w \mathbf{f}$, and we claim that one has $\mathcal{P}_{\mathbf{f}^{\prime}} \cap \mathcal{P}_{w \mathbf{f}}=\mathcal{P}_{\mathbf{f}^{\prime} \cup w \mathbf{f}}$ as closed subgroups of $L G$. This implies (i) because $\mathcal{P}_{\Omega}$ is the strictly pro-algebraic $\mathbf{Z}$-group scheme given by the functor $R \mapsto \mathcal{G}_{\Omega}(R \llbracket \varpi \rrbracket)$, and satisfies the asserted properties by Lemma 4.2.7 and Remark 4.2.8. It remains to prove the claim. Recall that, in fact for any $\Omega$, the group scheme $\mathcal{G}_{\Omega}$ is constructed from the rational group law on the big open cell

$$
\mathcal{G}_{\Omega}^{o}:=\mathcal{U}_{\Omega}^{-} \times \mathcal{T} \times \mathcal{U}_{\Omega}^{+} \rightarrow \mathcal{G}_{\Omega}, \quad\left(u^{-}, t, u^{+}\right) \mapsto u^{-} \cdot t \cdot u^{+},
$$

where $\mathcal{T}:=T \otimes_{\mathbf{Z}} \mathbf{Z} \llbracket \varpi \rrbracket$, and $\mathcal{U}_{\Omega}^{ \pm}$are split unipotent algebraic $\mathbf{Z} \llbracket \varpi \rrbracket$-group schemes, cf. [PZ13, Section 4.2.2] applied with $\mathbf{Z} \llbracket \varpi \rrbracket$ as a base ring. Here $\mathcal{G}_{\Omega}^{o} \subset \mathcal{G}_{\Omega}$ is an open subscheme which is fiberwise dense over $\mathbf{Z} \llbracket \varpi \rrbracket$ and carries a rational group law compatible with the group law on $\mathcal{G}_{\Omega}$. Denote by $\mathcal{P}_{\Omega}^{o}$ the functor $R \mapsto \mathcal{G}_{\Omega}^{o}(R \llbracket \varpi \rrbracket)$. We claim that $\mathcal{P}_{\Omega}^{o} \subset \mathcal{P}_{\Omega}$ is relatively representable by an open immersion which is fiberwise dense over $\mathbf{Z}$. Indeed, the same construction as in (4.2.5) applies so that $\mathcal{P}_{\Omega}^{o}=\lim _{i \geqslant 0} \mathcal{P}_{\Omega, i}^{o}$ is an inverse limit of smooth affine $\mathbf{Z}$-schemes. Note that each $\mathcal{P}_{\Omega, i}^{o} \subset \mathcal{P}_{\Omega, i}$ is an open subscheme which is fiberwise dense over $\mathbf{Z}$. In passing to the limit, we need to make sure that the open subsets are not getting too small. Since $\mathcal{P}_{\Omega, i}^{o}$ contains the unit section, the construction in the proof of Proposition A.9 below applies to show that $\operatorname{ker}\left(\mathcal{P}_{\Omega, i}^{o} \rightarrow \mathcal{P}_{\Omega, i-1}^{o}\right)=\operatorname{ker}\left(\mathcal{P}_{\Omega, i} \rightarrow \mathcal{P}_{\Omega, i-1}\right)$ for all $i \geqslant 1$ (the left hand side are by definition the sections which map to the unit section). Here we used that under the open immersion $\mathcal{G}_{\Omega}^{o} \subset \mathcal{G}_{\Omega}$ the associated vector bundles (A.11) agree. It follows that $\operatorname{ker}\left(\mathcal{P}_{\Omega}^{o} \rightarrow \mathcal{P}_{\Omega, 0}^{o}\right)=\operatorname{ker}\left(\mathcal{P}_{\Omega} \rightarrow \mathcal{P}_{\Omega, 0}\right)$ which implies the claim. Now from the construction of $\mathcal{G}_{\Omega}^{o}$ it is immediate that $\mathcal{P}_{\mathbf{f}^{\prime}}^{o} \cap \mathcal{P}_{w \mathbf{f}}^{o}=\mathcal{P}_{\mathbf{f}^{\prime} \cup w \mathbf{f}}^{o}$. Thus, the natural closed immersion $\mathcal{P}_{\mathbf{f}^{\prime} \cup w \mathbf{f}} \subset \mathcal{P}_{\mathbf{f}^{\prime}} \cap \mathcal{P}_{w \mathbf{f}}$ is an equality over the open and fiberwise dense subscheme $\mathcal{P}_{\mathbf{f}^{\prime} \cup w \mathbf{f}}^{o}$. This immediately implies $\mathcal{P}_{\mathbf{f}^{\prime} \cup w \mathbf{f}}=\mathcal{P}_{\mathbf{f}^{\prime}} \cap \mathcal{P}_{w \mathbf{f}}$, and shows (i).

Part (ii) is proven the same way as [Ric16a, Corollary 3.14]. In the reference, the base is a discrete valuation ring, but the same argument works over the Dedekind $\operatorname{ring} \mathbf{Z}$ as well. Also we note that for $i \gg 0$, the split pro-unipotent kernel $U_{i}=\operatorname{ker}\left(\mathcal{P}^{\prime} \rightarrow \mathcal{P}_{i}^{\prime}\right)$ lies in $\mathcal{P}_{w}^{\prime}$, so that $\overline{\mathcal{P}}_{w, i}^{\prime}:=\left(\mathcal{P}_{w}^{\prime} / U_{i}\right)^{\text {ét }} \subset \mathcal{P}_{i}^{\prime}$ is fiberwise connected, cellular and smooth over $\mathbf{Z}$. 
Part (iii) is deduced the same way as for example in [Ric13, Proposition 2.8(i)] (which is over fields) using the existence of Demazure resolutions.

Now assume that $\mathbf{f}, \mathbf{f}^{\prime} \subset \mathscr{A}$ are contained in the closure of the base alcove $\mathbf{a}_{0}$, and consider the double classes $W_{\mathbf{f}^{\prime}} \backslash W / W_{\mathbf{f}}$ equipped with its length function $l$ and partial order $\leqslant$ as in (4.2.15) above. By Lemma 4.3.7, there is a presentation of the underlying reduced ind-scheme

$$
\mathrm{Fl}_{\text {red }}=\operatorname{colim}_{w} \mathrm{Fl}^{\leqslant w}
$$

where $w$ runs over the partial ordered set $W_{\mathbf{f}^{\prime}} \backslash W / W_{\mathbf{f}}$. The following proposition equips Fl with a cellular stratification.

Proposition 4.3.9. Assume $\mathbf{f}^{\prime}=\mathbf{a}_{0}$, and let $w \in W / W_{\mathbf{f}}$.

(i) There is an isomorphism of schemes $\mathrm{Fl}^{w} \simeq \mathbf{A}_{\mathbf{Z}}^{l(w)}$ where $l(w) \in \mathbf{Z}_{\geqslant 0}$ is the length function on $W / W_{\mathbf{f}}$.

(ii) The quotient map $L G \rightarrow \mathrm{Fl}$ has sections over $\mathrm{Fl}^{w}$.

Proof. For any affine root $\alpha=a+k \in \mathcal{R}$, there is the root homomorphism $u_{\alpha}: \mathbf{G}_{a} \rightarrow L G, x \mapsto u_{a}\left(x z^{k}\right)$, see for example [dCHL18, Section 3.5]. The reference is written over a field, but the same formulas work over $\mathbf{Z}$ as well, cf. also [Con14, Section 5.1] for root subgroups in the relative set-up. Then $u_{\alpha}$ is a closed immersion, and we let $U_{\alpha} \subset L G$ be its image which is a closed $\mathbf{Z}$-subgroup scheme of $L G$ isomorphic to the additive group $\mathbf{G}_{a}=\mathbf{A}_{\mathbf{Z}}^{1}$. We consider the map

$$
\pi: \prod_{\alpha} U_{\alpha} \rightarrow \mathrm{Fl}^{w}, \quad\left(u_{\alpha}\right)_{\alpha} \mapsto\left(\prod_{\alpha} u_{\alpha}\right) \cdot \dot{w} \cdot e,
$$

where the product (taken in any fixed order) ranges over all affine roots $\alpha \in \mathcal{R}$ such that $\left.(w \alpha)\right|_{\mathbf{a}_{0}}$ takes positive values and $\left.\alpha\right|_{\mathbf{f}}$ takes negative values. We claim that $\pi$ is an isomorphism of $\mathbf{Z}$-schemes. Indeed, as source and target are smooth $\mathbf{Z}$-schemes of finite type, the fibral isomorphism criterion from [SGA03, I.5, Proposition 5.7] reduces us to prove that $\pi \otimes k$ is an isomorphism for any (prime) field $k$. This is well known, cf. for example [dCHL18, Proposition 3.7.4, (3.32)]. Also note that the number of such roots as above is $l(w)$ for the length taken on $W / W_{\mathbf{f}}$. This implies (i). Part (ii) also follows because the sections are given by $\pi^{-1}$ composed with the closed immersion $\prod_{\alpha} U_{\alpha} \rightarrow L G,\left(u_{\alpha}\right)_{\alpha} \mapsto\left(\prod_{\alpha} u_{\alpha}\right) \cdot \dot{w}$. This concludes the proof of the proposition.

REMARK 4.3.11. Proposition 4.3.9(ii) fails if $\mathbf{f}^{\prime}$ is not an alcove, that is, $\mathcal{P}_{\mathbf{f}^{\prime}}$ strictly contains the standard Iwahori $\mathcal{B}$. Indeed, if the map $L G \rightarrow \mathrm{Fl}$ has a section 
over $\mathrm{Fl}^{w}$, then $\mathrm{Fl}^{w}$ must necessarily be affine (because $L G$ is ind-affine). However, whenever $\mathbf{f}^{\prime}$ is not an alcove, there exists $w \in W$ such that $\mathrm{Fl}^{w}$ is not affine. As an example consider $G=\mathrm{GL}_{2}$, and take $\mathbf{f}^{\prime}=\mathbf{f}=0$. Then, for $\mu=(1,0) \in X_{*}(T)_{+}$, we have $\mathrm{Gr}_{G}^{\leqslant \mu}=\mathbf{P}_{\mathbf{Z}}^{1}$.

Corollary 4.3.12. Let $\mathrm{Fl}^{+}:=\bigsqcup_{w \in W_{\mathrm{f}^{\prime} \backslash W / W_{\mathrm{f}}}} \mathrm{Fl}^{w}$. Then the inclusion $\iota: \mathrm{Fl}^{+} \rightarrow$ Fl is a cellular stratified ind-scheme in the sense of Definition 3.1.5. For $\mathbf{f}^{\prime}=\mathbf{a}_{0}$, we refer to this stratification on $\mathrm{Fl}$ as the Iwahori stratification.

Proof. Each $\mathcal{P}^{\prime}$-orbit $\mathrm{Fl}^{w}$ is a smooth $\mathbf{Z}$-scheme with geometrically connected fibers by Lemma 4.3.7(ii), and it decomposes in Iwahori orbits as

$$
\mathrm{Fl}^{w}=\bigsqcup_{v \in W_{\mathbf{f}^{\prime}} w W_{\mathbf{f}} / W_{\mathbf{f}}} \mathrm{Fl}^{v},
$$

where each $\mathrm{Fl}^{v}$ is isomorphic to an affine space, cf. Proposition 4.3.9(i). Thus each $\mathrm{Fl}^{w}$ is a cellular $\mathbf{Z}$-scheme. Further, by (4.3.8) together with Lemma 4.3.7(iii), the map $\iota$ is bijective on the underlying topological spaces. As each restriction $\left.\iota\right|_{\mathrm{Fl}^{w}}$ is the composition $\mathrm{Fl}^{w} \subset \mathrm{Fl}^{\leqslant w} \subset \mathrm{Fl}$ of a quasicompact open immersion followed by a closed immersion, it is a quasicompact immersion. Also we have for the closure $\overline{\mathrm{Fl}}^{w}=\mathrm{Fl}^{\leqslant w}$ by Lemma 4.3.7(ii). This implies the corollary.

Now assume further that $\mathcal{B} \subset \mathcal{P}^{\prime} \subset \mathcal{P}$, that is, $\mathbf{f}$ is contained in the closure of $\mathbf{f}^{\prime}$. We end this subsection by investigating the behavior of the Iwahori stratification under the projection $\pi: \mathrm{Fl}_{\mathbf{f}^{\prime}} \rightarrow \mathrm{Fl}_{\mathbf{f}}$. The following proposition relates to Lemmas 3.1.19 and 3.1.20.

PROPOSITION 4.3.13. (i) The projection $\pi: \mathrm{Fl}_{\mathbf{f}^{\prime}} \rightarrow \mathrm{Fl}_{\mathbf{f}}$ is representable by a smooth proper surjective map which is étale-locally on the target isomorphic to the projection $\mathcal{P} / \mathcal{P}^{\prime} \times \mathrm{Fl}_{\mathbf{f}} \rightarrow \mathrm{Fl}_{\mathbf{f}}$.

(ii) The induced map on the Iwahori stratifications $\pi^{+}: \mathrm{Fl}_{\mathbf{f}^{\prime}}^{+} \rightarrow \mathrm{Fl}_{\mathbf{f}}^{+}$is a Tate map, and admits a section $s^{+}: \mathrm{Fl}_{\mathbf{f}}^{+} \rightarrow \mathrm{Fl}_{\mathbf{f}^{\prime}}^{+}$which is an open and closed immersion.

Proof. For (i) we refer to the proof of [HR18c, Lemma 4.9(i)] for details. For (ii), first let $\mathbf{f}^{\prime}=\mathbf{a}_{0}$ be the base alcove, and abbreviate $\mathrm{Fl}=\mathrm{Fl}_{\mathbf{f}^{\prime}}$. For $w \in W / W_{\mathbf{f}}$, we have

$$
\left(\pi^{+}\right)^{-1}\left(\mathrm{Fl}_{\mathbf{f}}^{w}\right)=\bigsqcup_{v \in w W_{\mathrm{f}}} \mathrm{Fl}^{v}
$$

There exists a unique element $w_{\min } \in w W_{\mathrm{f}}$ of minimal length, cf. for example [Ric13, Lemma 1.6(i)]. Thus, every $v \in w W_{\mathbf{f}}$ can be written uniquely in the 
form $v=v_{0} \cdot w_{\min }$. It follows from (4.3.10) that the restriction $\left.\pi^{+}\right|_{\mathrm{Fl}^{v}}: \mathrm{Fl}^{v} \rightarrow \mathrm{Fl}_{\mathbf{f}}^{w}$ has the structure of a relative affine space of relative dimension $l\left(v_{0}\right)$. In particular, $\pi^{+}$is a Tate map, and $\left.\pi^{+}\right|_{\mathrm{Fl}^{w_{\text {min }}}}$ is an isomorphism. The desired section is given by

$$
s^{+}: \mathrm{Fl}_{\mathbf{f}}^{+}=\bigsqcup_{w \in W / W_{\mathbf{f}}} \mathrm{Fl}_{\mathbf{f}}^{w} \stackrel{\simeq}{\longleftarrow} \bigsqcup_{w \in W / W_{\mathbf{f}}} \mathrm{Fl}^{w_{\min }} \subset \bigsqcup_{w \in W} \mathrm{Fl}^{w}=\mathrm{Fl}^{+} .
$$

The case of more general facets $\mathbf{f}^{\prime}$ is reduced to this case by considering the projections $\mathrm{Fl}_{\mathbf{a}_{0}} \rightarrow \mathrm{Fl}_{\mathbf{f}^{\prime}} \rightarrow \mathrm{Fl}_{\mathbf{f}}$. This implies (ii), and proves the proposition.

EXAMPLE 4.3.14. Let $s \in \mathbb{S}$ be a simple affine reflection. Then there is a unique facet $\mathbf{f}_{s}$ of maximal dimension in the closure of $\mathbf{a}_{0}$ such that $s\left(\mathbf{f}_{s}\right)=\mathbf{f}_{s}$, that is, $W_{\mathbf{f}_{s}}$ is the subgroup generated by $s$. We specialize Proposition 4.3.13 to the case $\pi: \mathrm{Fl} \rightarrow \mathrm{Fl}_{\mathbf{f}_{s}}$ so that $\mathcal{B}=\mathcal{P} \subset \mathcal{P}^{\prime}=\mathcal{P}_{\mathbf{f}_{s}}$. In this case, the map $\pi$ has general fiber $\left(\mathcal{P}_{\mathbf{f}_{s}} / \mathcal{B}\right)^{\text {ét }}=\mathbf{P}_{\mathbf{Z}}^{1}$. If $w=v \cdot s$ is a reduced decomposition so that $v=w_{\min }$, then

$$
\left(\pi^{+}\right)^{-1}\left(\mathrm{Fl}_{\mathbf{f}_{s}}^{v}\right)=\mathrm{Fl}^{v} \sqcup \mathrm{Fl}^{v s} .
$$

Here $\left.\pi^{+}\right|_{\mathrm{Fl}^{v}}$ is an isomorphism, and $\left.\pi^{+}\right|_{\mathrm{Fl}^{v s}}$ is an affine space of relative dimension 1 .

4.4. Changing the base scheme. Let $S$ be any nonempty scheme. We change notation, and let $G$ be a split reductive $S$-group scheme, that is, a smooth $S$ affine $S$-group whose fibers are connected reductive groups, and which admits a maximal split torus, cf. [Con14, Definition 5.1.1] for a precise definition. Recall that by the Isomorphism Theorem [Con14, Theorem 6.1.17] the group $G$ is already defined over $\mathbf{Z}$, that is, there exists a Chevalley group $G_{\mathbf{Z}}$ such that $G=G_{\mathbf{Z}} \times_{\operatorname{Spec} \mathbf{Z}} S$ (we fix the isomorphism). We also fix $T_{\mathbf{Z}} \subset B_{\mathbf{Z}} \subset G_{\mathbf{Z}}$ as in (4.1.1), and let $T \subset B \subset G$ be the base change to $S$. We denote by $\mathscr{A}$ (respectively $W)$ the apartment (respectively Iwahori-Weyl group) associated with $\left(G_{\mathbf{Z}}, T_{\mathbf{Z}}\right)$. The definitions and constructions from Sections 4.2 and 4.3 generalize to general base schemes as follows.

The loop group $L G$ is the functor given by $L G(R)=G(R((\varpi)))$ for $\operatorname{Spec}(R)$ $\in \operatorname{AffSch}_{S}$. Clearly, we have $L G=L G_{\mathbf{Z}} \times_{\operatorname{Spec}(\mathbf{Z})} S$. Likewise, for any facet $\mathbf{f} \subset \mathscr{A}$ the parahoric subgroup $\mathcal{P}_{\mathbf{f}} \subset L G$ is defined by base change from $\mathbf{Z}$. In particular, $\mathcal{P}_{\mathbf{f}}=\lim _{i \geqslant 0} \mathcal{P}_{\mathbf{f}, i}$ is a strictly pro-algebraic $S$-group with geometrically connected fibers. The partial affine flag variety $\mathrm{Fl}_{\mathbf{f}}$ is the étale sheaf associated with the functor $R \mapsto L G(R) / \mathcal{P}_{\mathbf{f}}(R)$ for $\operatorname{Spec}(R) \in \operatorname{AffSch}_{S}$. Since sheafification commutes with base change, we see that $\mathrm{Fl}_{\mathbf{f}}=\mathrm{Fl}_{\mathbf{f}, \mathbf{Z}} \times{ }_{\operatorname{Spec}(\mathbf{Z})} S \rightarrow S$ is the base change from $\mathbf{Z}$ as well, and in particular ind-projective.

Let $\mathbf{f}, \mathbf{f}^{\prime} \subset \mathscr{A}$ be two facets, and abbreviate $\mathcal{P}^{\prime}:=\mathcal{P}_{\mathbf{f}^{\prime}}$ and $\mathrm{Fl}:=\mathrm{Fl}_{\mathbf{f}}$. For any $S$-scheme $T$, we write $L G(T):=\operatorname{Hom}_{S}(T, L G)$, and likewise for Fl. 
Definition 4.4.1. For $w \in W_{\mathbf{f}^{\prime}} \backslash W / W_{\mathbf{f}}$, the Schubert scheme $\mathrm{Fl}^{\leqslant w}={ }^{\mathbf{f}^{\prime}} \mathrm{Fl}_{\mathbf{f}}^{\leqslant w}$ over $S$ is the scheme-theoretic image of the map

$$
\mathcal{P}^{\prime} \rightarrow \mathrm{Fl}, \quad p^{\prime} \mapsto p^{\prime} \cdot \dot{w} \cdot e,
$$

where $e \in \operatorname{Fl}(S)$ is the base point, and $\dot{w} \in L G(S)$ is the image of a representative of $w$ under the map $L G(\mathbf{Z}) \rightarrow L G(S)$.

As in Definition 4.3.4 one sees that $\mathrm{Fl}^{\leqslant w} \subset \mathrm{Fl}$ defines a closed subscheme whose underlying topological space coincides with the closure of the topological image of (4.4.2).

Proposition 4.4.3. Let $\mathrm{Fl}_{\mathbf{Z}}^{\leqslant w} \subset \mathrm{Fl}_{\mathbf{Z}}$ be the Schubert scheme over $\mathbf{Z}$. Then the natural closed immersion $\mathrm{Fl}^{\leqslant w} \subset \mathrm{Fl}_{\mathbf{Z}}^{\leqslant w} \times_{\operatorname{Spec}(\mathbf{Z})} S$ is a Nil thickening.

Proof. We first note that the proposition is obvious whenever $S \rightarrow \operatorname{Spec}(\mathbf{Z})$ is flat because scheme-theoretic images along quasicompact maps commute with flat base change, cf. [Sta17, Tag 01R8]. If $S \rightarrow \operatorname{Spec}(\mathbf{Z})$ is not necessarily flat, we have to show that $\mathrm{Fl}^{\leqslant w} \subset \mathrm{Fl}_{0}^{\leqslant w} \times_{\operatorname{Spec}(\mathbf{Z})} S$ is an equality on topological spaces. By functoriality of the scheme-theoretic image [Sta17, Tag 01R9], for every field $\operatorname{Spec}(k) \rightarrow S$ we have closed immersions $\mathrm{Fl}_{k}^{\leqslant w} \subset \mathrm{Fl}^{\leqslant w} \times_{\operatorname{Spec}(S)} \operatorname{Spec}(k) \subset$ $\mathrm{Fl}_{\mathbf{Z}}^{\leqslant w} \times \operatorname{Spec(\mathbf {Z})} \operatorname{Spec}(k)$ where $\mathrm{Fl}_{k}^{\leqslant w}$ denotes the Schubert variety over $k$. Hence, Lemma 4.3.6 implies the claim.

The canonical closed immersion

$$
\operatorname{colim}_{w} \mathrm{Fl}^{\leqslant w} \hookrightarrow \mathrm{Fl},
$$

is a Nil thickening, and hence an isomorphism on the underlying reduced loci. In particular, (4.4.4) induces an equivalence on the categories of motives. For completeness, we remark that the Schubert scheme $\mathrm{Fl}^{\leqslant w}$ is nonreduced if $S$ is nonreduced so that we need to pass to the underlying reduced loci on both sides in (4.4.4) to get an isomorphism.

For $w \in W_{\mathbf{f}^{\prime}} \backslash W / W_{\mathbf{f}}$, we define $\mathrm{Fl}^{w}$ as the étale sheaf image of (4.4.2). Since sheaf-theoretic images commute with base change, we see that $\mathrm{Fl}^{w}$ is the base change from $\mathbf{Z}$. In particular, $\mathrm{Fl}^{w} \subset \mathrm{Fl}^{\leqslant w}$ is an open subscheme which is smooth, fiberwise dense and geometrically connected over $S$. Now all results of Lemma 4.3.7, Proposition 4.3.9, Corollary 4.3.12, Proposition 4.3.13 and Example 4.3.14 translate literally to the general context by base change $S \rightarrow \operatorname{Spec}(\mathbf{Z})$. 


\section{Mixed Tate motives on affine flag varieties}

Notation 5.0.1. Throughout Section 5, we assume $S$ is as in Notation 2.0.1 and satisfies furthermore the Beilinson-Soulé vanishing conjecture as in (3.2.2). We also assume $S$ admits an $\ell$-adic realization functor in the sense of Remark 3.2.9. Examples include finite fields $\mathbf{F}_{q}$, function fields $\mathbf{F}_{q}(t)$, number fields $F$, and their algebraic (separable/perfect) closures. Further examples are the ring of algebraic integers $\mathcal{O}_{F}$, and smooth curves over finite fields.

W fix $T \subset B \subset G$ over $S$ as in Section 4.4. We let $\mathbf{f}, \mathbf{f}^{\prime} \subset \mathscr{A}$ be two facets which are contained in the closure of the base alcove $\mathbf{a}_{0}$. Their associated parahoric groups are denoted $\mathcal{P}_{\mathbf{f}}, \mathcal{P}_{\mathbf{f}^{\prime}} \subset L G$. In the following, we use Section 4.4 without explicit reference in order to apply the results from Sections 4.2-4.3. Throughout, $w$ denotes an element in $W_{\mathbf{f}^{\prime}} \backslash W / W_{\mathbf{f}}$; thus, $w$ parametrizes the orbits of the left action of the pro-algebraic $S$-group $\mathcal{P}_{\mathbf{f}^{\prime}}$ on the ind-scheme $\mathrm{Fl}_{\mathbf{f}}$. The inclusion of such an orbit is denoted $\iota_{w}: \mathrm{Fl}_{\mathbf{f}}^{w} \rightarrow \mathrm{Fl}_{\mathbf{f}}$. By Corollary 4.3.12, these orbits yield a cellular stratification which is denoted

$$
\iota: \bigsqcup_{w \in W_{\mathbf{f}^{\prime}} \backslash W / W_{\mathbf{f}}} \mathrm{Fl}_{\mathbf{f}}^{w} \rightarrow \mathrm{Fl}_{\mathbf{f}} .
$$

In this section, we apply the results of Section 3 to partial affine flag varieties and obtain a category of Tate motives on the double quotient $\mathcal{P}_{\mathbf{f}^{\prime}} \backslash L G / \mathcal{P}_{\mathbf{f}}$. Also, if $\mathbf{f}^{\prime}=\mathbf{f}$, then we obtain an abelian subcategory of mixed motives on $\mathcal{P}_{\mathbf{f}} \backslash L G / \mathcal{P}_{\mathbf{f}}$. The latter category contains the intersection motives $\mathrm{IC}_{w} \in \mathrm{DM}\left(\mathcal{P}_{\mathbf{f}} \backslash L G / \mathcal{P}_{\mathbf{f}}\right)$ that will be used in Section 6 to construct the intersection motive of the moduli stack of $G$-shtukas.

\subsection{Whitney-Tate stratifications on partial affine flag varieties.}

THEOREM 5.1.1. The stratification (5.0.2) is a Whitney-Tate stratification.

In [SW18, Proposition 4.10], Soergel and Wendt prove the analogous statement for the Borel orbit stratification in partial flag varieties over fields. Thus, Theorem 5.1.1 generalizes their result in three ways. We work with infinitedimensional partial affine flag varieties in which the finite-dimensional partial flag varieties can be embedded compatibly with the Iwahori respectively Borel stratifications. We allow the stratification into $\mathcal{P}_{\mathbf{f}^{\prime}}$-orbits instead of merely Iwahori orbits. We work over more general base schemes; for example $S=\operatorname{Spec}(\mathbf{Z})$ is allowed. The last feature will be used in [RS19] to transfer the purity of the intersection motives $\mathrm{IC}_{w}$ from the case that $S$ has positive characteristic to the 
case that $S$ has characteristic zero. The proof of Theorem 5.1.1 proceeds in three steps; the first two, that is, the case of the Iwahori stratification, are an extension of the arguments [SW18, Proposition 4.10] to the affine flag variety. We also point out that Habibi [Hab11, Corollary 5.4.12] has shown that the motive of affine Schubert varieties $\mathrm{Fl}_{\mathbf{f}} \leqslant w$ is a Tate motive provided that the Demazure resolution is semismall.

Proof of Theorem 5.1.1. First step: $\mathbf{f}^{\prime}=\mathbf{f}=\mathbf{a}_{0}$ is the base alcove. Write $\mathrm{Fl}:=\mathrm{Fl}_{\mathbf{a}_{0}}$, and $\mathcal{B}:=\mathcal{P}_{\mathbf{a}_{0}}$ for the Iwahori group. We have to show $\iota^{!} \iota_{!} 1 \in \operatorname{DTM}\left(\mathrm{Fl}^{+}\right)$, that is, the restriction to each stratum $\mathrm{Fl}^{w}$ is a Tate motive. By induction on the length $l(w)$, we show that

$$
\iota^{!}\left(\iota_{w}\right) ! 1 \in \mathrm{DTM}\left(\mathrm{Fl}^{+}\right) .
$$

If $l(w)=0$, then necessarily $w \in \operatorname{Stab}_{\mathbf{a}_{0}}$ by (4.2.13) so that $\iota_{w}$ is a closed immersion.

If $l(w)>0$, there is a decomposition $w=v s$ for some simple reflection $s \in \mathbb{S}$ such that $l(v)=l(w)-1$. As in Example 4.3.14 we denote by $\mathcal{B} \subset \mathcal{P}_{\mathbf{f}_{s}} \subset L G$ the parahoric subgroup associated with $s \in \mathbb{S}$, and by $\mathrm{Fl}_{\mathbf{f}_{s}}$ the corresponding partial affine flag variety. The projection $\pi: \mathrm{Fl} \rightarrow \mathrm{Fl}_{\mathbf{f}_{s}}$ is smooth and proper with fiber étale-locally isomorphic to $\left(\mathcal{P}_{\mathbf{f}_{s}} / \mathcal{B}\right)^{\text {ét }}=\mathbf{P}_{S}^{1}$, and the induced map on the Iwahori stratifications $\pi^{+}: \mathrm{Fl}^{+} \rightarrow \mathrm{Fl}_{\mathbf{f}_{s}}^{+}$is a Tate map which admits a section, cf. Proposition 4.3.13 and Example 4.3.14. We consider the commutative diagram

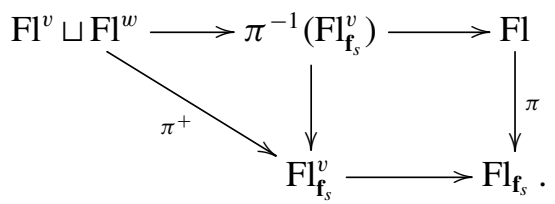

Then $\left.\pi^{+}\right|_{\mathrm{Fl}^{v}}$ is an isomorphism, and $\left.\pi^{+}\right|_{\mathrm{Fl}}$ is a relative 1-dimensional affine space. The localization sequence for $\mathrm{Fl}^{v} \rightarrow \pi^{-1}\left(\mathrm{Fl}_{\mathbf{f}_{s}}^{v}\right) \leftarrow \mathrm{Fl}^{w}$ therefore gives a fiber sequence

$$
\left(\iota_{w}\right) ! 1 \rightarrow \pi^{*} \pi_{*}\left(\iota_{v}\right) ! 1 \rightarrow\left(\iota_{v}\right) ! 1 .
$$

If we apply $\iota^{!}$, the right hand term lies in $\operatorname{DTM}\left(\mathrm{Fl}^{+}\right)$by induction, the middle term therefore also, by Lemma 3.1.18 and the smoothness of $\pi$ (which allows to replace $\pi^{*}$ by $\pi^{!}$). Hence the left hand term is also a Tate motive.

Second step: $\mathbf{f}^{\prime}=\mathbf{a}_{0}$ is the base alcove, and $\mathbf{f}$ arbitrary (but contained in the closure of $\mathbf{a}_{0}$ ). We consider the canonical projection $\pi: \mathrm{Fl}_{\mathbf{a}_{0}} \rightarrow \mathrm{Fl}_{\mathbf{f}}$ where both affine flag varieties are equipped with the Iwahori stratification. By Proposition 4.3.13 and the first step, we may apply Lemma 3.1.19 to $\pi$ and conclude. 
Third step: $\mathbf{f}^{\prime}, \mathbf{f}$ are arbitrary. We have to show that

$$
M:=\left(\iota_{w}\right)^{!}\left(\iota_{v}\right) ! 1 \in \operatorname{DTM}\left(\mathrm{Fl}_{\mathbf{f}}^{w}\right),
$$

for each $v, w \in W_{\mathbf{f}^{\prime}} \backslash W / W_{\mathbf{f}}$, where $\iota_{w}=\left.\iota\right|_{\mathrm{F}_{\mathrm{f}}^{w}}$ (respectively $\iota_{v}=\left.\iota\right|_{\mathrm{F}_{\mathrm{f}}^{v}}$ ). Note that $M$ is $\mathcal{P}_{\mathbf{f}^{\prime}}$-equivariant because $\iota_{w}$ and $\iota_{v}$ are $\mathcal{P}_{\mathbf{f}^{\prime}}$-equivariant. By Step 2 ) we know that !-restricting further to the Iwahori orbits gives Tate motives, and we use the equivariance to prove (5.1.3) as follows: By Lemma 4.3.7(i) and (ii), the map $\mathcal{P}^{\prime}:=\mathcal{P}_{\mathbf{f}^{\prime}} \rightarrow \mathrm{Fl}_{\mathbf{f}}^{w}, p^{\prime} \mapsto p^{\prime} \cdot \dot{w} \cdot e$ induces an isomorphism $\mathrm{Fl}_{\mathbf{f}}^{w}=\mathcal{P}^{\prime} / \mathcal{P}_{w}^{\prime}=$ $\mathcal{P}_{i}^{\prime} / \overline{\mathcal{P}}_{w, i}^{\prime}$ for $i \gg 0$ where both $\mathcal{P}_{i}^{\prime}$ and $\overline{\mathcal{P}}_{w, i}^{\prime}$ are fiberwise connected and cellular. Let $e_{w}: S \rightarrow \mathrm{Fl}_{\mathrm{f}}^{w}$ be the inclusion of the base point. By Proposition 3.1.23, $M$ is Tate if and only if $\left(e_{w}\right)^{!} M$ is a Tate motive on $S$. But this holds true since $M$ is a Tate motive with respect to the Iwahori stratification.

5.2. Tate motives on partial affine flag varieties. Given the cellular Whitney-Tate stratification of $\mathrm{Fl}_{\mathbf{f}}$ by $\mathcal{P}_{\mathbf{f}^{\prime}}$-orbits we can apply Definition and Lemma 3.1.11 to get a well-defined subcategory of stratified Tate motives

$$
\operatorname{DTM}\left(\mathrm{Fl}_{\mathbf{f}}\right) \subset \operatorname{DM}\left(\mathrm{Fl}_{\mathbf{f}}\right) .
$$

It is the subcategory generated (by arbitrary shifts and colimits) by the objects $\left(\iota_{w}\right) ! 1_{\mathrm{F}_{\mathrm{f}}^{w}}(n)$ for $n \in \mathbf{Z}, w \in W_{\mathbf{f}^{\prime}} \backslash W / W_{\mathbf{f}}$. This category admits the following characterization, similarly to [Soe00, Lemma 3.2.1]:

PROPOSITION 5.2.2. We equip all categories with the Iwahori stratification.

(i) The category $\mathrm{DTM}\left(\mathrm{Fl}_{\mathbf{a}_{0}}\right)$ is the smallest cocomplete full subcategory of $\operatorname{DM}\left(\mathrm{Fl}_{\mathbf{a}_{0}}\right)$ which contains the twists of the unit motives supported at the base points $\{\tau\}$ for each $\tau \in \operatorname{Stab}_{\mathbf{a}_{0}}$ (cf. (4.2.13)), and which is stable under the operation $\pi_{s}^{*} \pi_{s, *}$ (equivalently $\pi_{s}^{!} \pi_{s, !}$ ) along the smooth proper projection maps $\pi_{s}: \mathrm{Fl}_{\mathbf{a}_{0}} \rightarrow \mathrm{Fl}_{\mathbf{f}_{s}}$ for all $s \in \mathbb{S}$ (in the notation of Example 4.3.14).

(ii) Consider $\pi: \mathrm{Fl}_{\mathbf{a}_{0}} \rightarrow \mathrm{Fl}_{\mathbf{f}}$. The functor $\pi_{!}=\pi_{*}: \operatorname{DTM}\left(\mathrm{Fl}_{\mathbf{a}_{0}}\right) \rightarrow \operatorname{DTM}\left(\mathrm{Fl}_{\mathbf{f}}\right)$ is well-defined and the images of the generators as in (i) generate the target category.

Proof. For (i), it is immediate from (5.1.2) that the generators $\left(\iota_{w}\right) ! 1$ (for $w \in W$ ) are obtained inductively by writing $w$ as a product of simple reflections as in (4.2.14). Part (ii) is immediate from Proposition 4.3.13(ii) and Example 3.1.17.

THEOREM 5.2.3. (i) The category $\mathrm{DTM}\left(\mathrm{Fl}_{\mathbf{f}}\right)$ admits a nondegenerate 'motivic' $t$-structure. Its heart is the abelian category of mixed stratified Tate motives

$\operatorname{MTM}\left(\mathrm{Fl}_{\mathbf{f}}\right) \subset \operatorname{DTM}\left(\mathrm{Fl}_{\mathbf{f}}\right)$. 
If in addition $S$ is irreducible, the simple objects in $\mathrm{MTM}\left(\mathrm{Fl}_{\mathbf{f}}\right)$ are precisely the intersection motives $\operatorname{IC}_{w}(n)$ for $n \in \mathbf{Z}, w \in W_{\mathbf{f}^{\prime}} \backslash W / W_{\mathbf{f}}$ (see (3.3.6)).

(ii) The restriction of the $\ell$-adic realization functor

$$
\rho_{\ell}: \operatorname{DM}\left(\mathrm{Fl}_{\mathbf{f}}\right) \rightarrow \mathrm{D}_{\text {ét }}\left(\mathrm{Fl}_{\mathbf{f}}, \mathbf{Q}_{\ell}\right),
$$

(cf. Synopsis 2.1.1(xvii), Theorem 2.4.2) to the subcategory $\mathrm{DTM}\left(\mathrm{Fl}_{\mathbf{f}}\right)$ is conservative. Moreover, for $M \in \mathrm{DTM}\left(\mathrm{Fl}_{\mathbf{f}}\right)$ the following are equivalent: (a) $M$ lies in $\operatorname{MTM}\left(\mathrm{Fl}_{\mathbf{f}}\right)$, and $(b) \rho_{\ell}(M)$ is a perverse sheaf. Finally, $\rho_{\ell}\left(\mathrm{IC}_{w}(n)\right)$ is the $\ell$-adic intersection complex normalized relative to $S$ for all $w, n \in \mathbf{Z}$.

Proof. For (i), we combine Corollary 3.2.6 and Theorem 3.3.8. Part (ii) is also immediate from (i) and Lemma 3.2.8.

5.3. Tate motives on double quotients of the loop group. We now turn to (Tate) motives on the prestack

$$
\mathcal{P}_{\mathbf{f}^{\prime}} \backslash L G / \mathcal{P}_{\mathbf{f}}
$$

Here we view (5.3.1) as a prestack in the sense of Section 2.2 where we may choose $\kappa=\omega$ to be the countable cardinal. By Proposition 2.2.25, its étale stackification $\left(\mathcal{P}_{\mathbf{f}^{\prime}} \backslash L G / \mathcal{P}_{\mathbf{f}}\right)^{\text {ét }}$ is given by the prestack which sends $T \in \operatorname{AffSch}_{S}$ to the groupoid $\left(\mathcal{P}_{\mathbf{f}^{\prime}} \backslash L G / \mathcal{P}_{\mathbf{f}}\right)^{\text {ét }}(T)$ of diagrams of ind-schemes $T \stackrel{a}{\leftarrow} P \stackrel{b}{\rightarrow}$ $L G$ where $a$ is an étale-locally trivial torsor under the pro-algebraic group $\left(\mathcal{P}_{\mathbf{f}^{\prime}} \times \mathcal{P}_{\mathbf{f}}\right) \times{ }_{S} T$ (in particular an affine scheme), and $b$ is equivariant for the action of this group. (Here 'groupoid' is understood in the sense of an ordinary category whose morphisms are invertible. We regard it as an $\infty$-groupoid in the natural way.) The following lemma shows that the category of motives does not change when viewing (5.3.1) either as a prestack or an honest stack.

LEMMA 5.3.2. (i) The stack $\left(\mathcal{P}_{\mathbf{f}^{\prime}} \backslash L G / \mathcal{P}_{\mathbf{f}}\right)^{\text {ét }}$ is a sheaf of groupoids for the fpqc topology.

(ii) Étale sheafification of prestacks (or alternatively, in the above description of

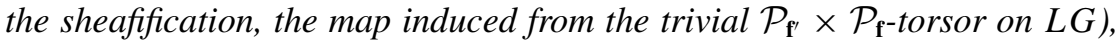
yields an equivalence of categories of motives

$$
\operatorname{DM}\left(\left(\mathcal{P}_{\mathbf{f}^{\prime}} \backslash L G / \mathcal{P}_{\mathbf{f}}\right)^{\text {ét }}\right) \stackrel{\simeq}{\longrightarrow} \operatorname{DM}\left(\mathcal{P}_{\mathbf{f}^{\prime}} \backslash L G / \mathcal{P}_{\mathbf{f}}\right) .
$$

In particular, $\operatorname{DM}\left(\mathcal{P}_{\mathbf{f}^{\prime}} \backslash L G / \mathcal{P}_{\mathbf{f}}\right)$ is also equivalent to either of the categories $\operatorname{DM}\left(\mathcal{P}_{\mathbf{f}^{\prime}} \backslash \mathrm{Fl}_{\mathbf{f}}\right), \operatorname{DM}\left(\mathrm{Fl}_{\mathbf{f}^{\prime}}^{\mathrm{op}} / \mathcal{P}_{\mathbf{f}}\right)$. 
Proof. For (i), let $T^{\prime} \rightarrow T$ be a faithfully flat map in AffSch $_{S}$. Let $T^{\prime} \leftarrow P^{\prime} \rightarrow$ $L G$ be an object in $\left(\mathcal{P}_{\mathbf{f}^{\prime}} \backslash L G / \mathcal{P}_{\mathbf{f}}\right)^{\text {et }}\left(T^{\prime}\right)$ together with a descent datum along $T^{\prime} \rightarrow T$. By effectivity of descent for affine schemes [Sta17, Tag 0244], the torsor $T^{\prime} \leftarrow P^{\prime}$ descends to a fpqc-locally trivial torsor $T \leftarrow P$ represented by affine schemes. The map $P^{\prime} \rightarrow L G$ descends as well because every ind-scheme is an fpqc sheaf, cf. Section A.1. By Proposition A.9 (cf. also Lemma 4.2.7 and Example A.12(iii(a))) every fpqc-locally trivial torsor under $\mathcal{P}_{\mathbf{f}^{\prime}} \times \mathcal{P}_{\mathbf{f}}$ is étalelocally trivial. Thus, $T \leftarrow P \rightarrow L G$ is an object of $\left(\mathcal{P}_{\mathbf{f}^{\prime}} \backslash L G / \mathcal{P}_{\mathbf{f}}\right)^{\text {ét }}(T)$. Part (ii) follows from Proposition 2.2.25 applied with $\tau=$ ét.

All the above also applies, by symmetry, to $\mathrm{Fl}_{\mathbf{f}^{\prime}}^{\text {op }}:=\left(\mathcal{P}_{\mathbf{f}^{\prime}} \backslash L G\right)^{\text {ét }}$ equipped with its stratification by orbits for the right $\mathcal{P}_{\mathbf{f}}$-action. Combining the WhitneyTate stratification with the group action, Definition 3.1.21 yields two full subcategories

$$
\operatorname{DTM}_{\mathcal{P}_{\mathbf{f}^{\prime}}}\left(\mathrm{Fl}_{\mathbf{f}}\right) \text { and } \quad \operatorname{DTM}_{\mathcal{P}_{\mathbf{f}}}\left(\mathrm{Fl}_{\mathbf{f}^{\prime}}^{\text {op }}\right) \subset \operatorname{DM}\left(\mathcal{P}_{\mathbf{f}^{\prime}} \backslash L G / \mathcal{P}_{\mathbf{f}}\right) .
$$

We now address this seeming asymmetry and also upgrade Theorem 5.2.3 to equivariant motives. Let $L G^{w}:=\mathcal{P}_{\mathbf{f}^{\prime}} w \mathcal{P}_{\mathbf{f}}$ as a closed subscheme of $L G$. Formally, $L G^{w}$ is the scheme-theoretic image of the map $\mathcal{P}_{\mathbf{f}^{\prime}} \times \mathcal{P}_{\mathbf{f}} \rightarrow L G,\left(p^{\prime}, p\right) \mapsto p^{\prime} \dot{w} p$ where $\dot{w} \in L G(S)$ is any representative. We write $\iota_{w}$ for all maps of prestacks stemming from the inclusion of $L G^{w} \subset L G$, in particular $\iota_{w}: \mathcal{P}_{\mathbf{f}^{\prime}} \backslash L G^{w} / \mathcal{P}_{\mathbf{f}} \rightarrow$ $\mathcal{P}_{\mathbf{f}^{\prime}} \backslash L G / \mathcal{P}_{\mathbf{f}}$ and $\iota_{w}: \mathcal{P}_{\mathbf{f}^{\prime}} \backslash \mathrm{Fl}_{\mathbf{f}}^{w} \rightarrow \mathcal{P}_{\mathbf{f}^{\prime}} \backslash \mathrm{Fl}_{\mathbf{f}}$.

THEOREM 5.3.4. Let $S$ be irreducible.

(i) The functor $\iota_{w}^{!}: \operatorname{DM}\left(\mathcal{P}_{\mathbf{f}^{\prime}} \backslash L G / \mathcal{P}_{\mathbf{f}}\right) \rightarrow \operatorname{DM}\left(\mathcal{P}_{\mathbf{f}^{\prime}} \backslash L G^{w} / \mathcal{P}_{\mathbf{f}}\right)$ has a left adjoint denoted $\left(\iota_{w}\right)$ !

(ii) The two full categories in (5.3.3) both agree with the full subcategory of $\operatorname{DM}\left(\mathcal{P}_{\mathbf{f}^{\prime}} \backslash L G / \mathcal{P}_{\mathbf{f}}\right)$ generated (under shifts and colimits) by the objects $\left(\iota_{w}\right) ! 1(n)$ for $n \in \mathbf{Z}, w \in W_{\mathbf{f}^{\prime}} \backslash W / W_{\mathbf{f}}$. This category is denoted $\operatorname{DTM}\left(\mathcal{P}_{\mathbf{f}^{\prime}} \backslash L G / \mathcal{P}_{\mathbf{f}}\right)$.

(iii) The motivic t-structures on the categories $\mathrm{DTM}_{\mathcal{P}_{\mathrm{f}^{\prime}}}\left(\mathrm{Fl}_{\mathbf{f}}\right)$ and $\mathrm{DTM}_{\mathcal{P}_{\mathbf{f}}}\left(\mathrm{Fl}_{\mathbf{f}^{\prime}}^{\text {op }}\right)$ yield two $t$-structures on $\operatorname{DTM}\left(\mathcal{P}_{\mathbf{f}^{\prime}} \backslash L G / \mathcal{P}_{\mathbf{f}}\right)$. For $\mathbf{f}^{\prime}=\mathbf{f}$, these two $t$-structures agree in which case its heart is denoted

$$
\operatorname{MTM}\left(\mathcal{P}_{\mathbf{f}} \backslash L G / \mathcal{P}_{\mathbf{f}}\right) \subset \operatorname{DTM}\left(\mathcal{P}_{\mathbf{f}} \backslash L G / \mathcal{P}_{\mathbf{f}}\right) .
$$

It is generated by the intersection motives $\mathrm{IC}_{w}(n)$ for $n \in \mathbf{Z}, w \in W_{\mathbf{f}} \backslash W / W_{\mathbf{f}}$ from Theorem 5.2.3. Moreover, the forgetful functor $\operatorname{MTM}\left(\mathcal{P}_{\mathbf{f}} \backslash L G / \mathcal{P}_{\mathbf{f}}\right) \rightarrow$ $\operatorname{MTM}\left(\mathrm{Fl}_{\mathbf{f}}\right)$ is fully faithful, and induces a bijection on the isomorphism classes of simple objects. 
Proof. For (i), we may replace $L G / \mathcal{P}_{\mathbf{f}}$ and $L G^{w} / \mathcal{P}_{\mathbf{f}}$ by their étale sheafification (cf. Theorem 2.2.16), which are $\mathrm{Fl}_{\mathbf{f}}$ and its stratum $\mathrm{Fl}_{\mathbf{f}}^{w}$, respectively. These are ind-schemes, so we are done by Theorem 2.4.2 and Lemma 2.2.9.

For (ii), the objects $\left(\iota_{w}\right) ! 1(n)$ are independent of the role of $\mathbf{f}^{\prime}$ vs. $\mathbf{f}$. So it is enough to show that DTM $\mathcal{P}_{\mathcal{f}^{\prime}}\left(\mathrm{Fl}_{\mathbf{f}}\right)$ is generated by these objects as a subcategory of $\operatorname{DM}\left(\mathcal{P}_{\mathbf{f}^{\prime}} \backslash L G / \mathcal{P}_{\mathbf{f}}\right)$. By Proposition 3.1.27, which is applicable to $\mathcal{P}_{\mathbf{f}^{\prime}}$ acting on $\mathrm{Fl}_{\mathbf{f}}$ by Lemmas 4.2.7 and 4.3.7, we can reduce this claim to the case of the action of some algebraic quotient $\mathcal{P}_{\mathbf{f}^{\prime}, i}$ of $\mathcal{P}_{\mathbf{f}^{\prime}}$ on some subscheme (in $\mathrm{Sch}_{S}^{\mathrm{ft}}$ ) $\mathrm{Fl}_{\mathbf{f}, i} \subset \mathrm{Fl}_{\mathbf{f}}$. The stratification of $\mathrm{Fl}_{\mathbf{f}, i}$ by $\mathcal{P}_{\mathbf{f}^{\prime}, i}$-orbits is cellular by Corollary 4.3 .12 and the stabilizers are connected by Lemma 4.3.7(i), so we are done by Corollary 3.2.24.

For (iii), the $t$-structure on $\operatorname{DTM}_{\mathcal{P}_{\mathbf{f}^{\prime}}}\left(\mathrm{Fl}_{\mathbf{f}}^{w}\right)$ is characterized by the property that its $\leqslant 0$-part is generated by means of arbitrary colimits by the objects $1_{\mathrm{F}_{\mathrm{f}}^{w}}(n)\left[\operatorname{dim} \mathrm{Fl}_{\mathrm{f}}^{w}\right]$. Indeed, by Proposition 2.2.11 and Example 2.2.13(ii), we may replace $\mathcal{P}_{\mathbf{f}^{\prime}}$ by some algebraic quotient acting on $\mathrm{Fl}_{\mathbf{f}}^{w}$ and then apply Proposition 3.2.23. By construction of the glued $t$-structure in Corollary 3.2.6,

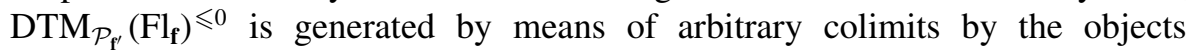
$\left(\iota_{w}\right) ! 1_{\mathrm{F}_{\mathbf{f}}^{w}}(n)\left[\operatorname{dim} \mathrm{Fl}_{\mathbf{f}}^{w}\right]$. Thus, for $\mathbf{f}^{\prime}=\mathbf{f}$, the two $t$-structures have the same $\leqslant 0$-part and therefore agree.

REMARK 5.3.5. For $\mathbf{f}^{\prime} \neq \mathbf{f}$ it may happen that $\operatorname{dim} \mathrm{Fl}_{\mathbf{f}}^{w} \neq \operatorname{dim} \mathrm{Fl}_{\mathbf{f}^{\prime}}^{\text {op } w}$, so the $t$-structures are different. As an example consider $G=\mathrm{GL}_{2}, \mathbf{f}^{\prime}=\mathbf{a}_{0}, \mathbf{f}=0$, and $w:=s_{1}$ the simple finite reflection. Then $\mathrm{Fl}_{\mathbf{f}}$ is the affine Grassmannian, and $\mathrm{Fl}_{\mathbf{f}}^{w}=\{e\}$ is the base point. On the other hand $\mathrm{Fl}_{\mathbf{f}^{\prime}}^{\text {op }}$ is the full affine flag variety, and $\mathrm{Fl}_{\mathbf{f}^{\prime}}^{\mathrm{op}, w}=\mathbf{P}_{S}^{1}$.

We end this section by pointing out the following corollary which is needed in Section 6. Specialize to $\mathbf{f}^{\prime}=\mathbf{f}=\{0\}$ being the base point, so that $W_{0} \backslash W / W_{0}=$ $X_{*}(T)_{+}$. The action of $L^{+} \mathbf{G}_{m, S}$ on $L G$ by changing the variable $\varpi$ preserves the subgroup $L^{+} G$, and thus gives an action on the double quotient $L^{+} G \backslash L G / L^{+} G$, and the affine Grassmannian $\mathrm{Gr}:=\left(L G / L^{+} G\right)^{\text {ét }}$.

Corollary 5.3.6. For each $\mu \in X_{*}(T)_{+}, n \in \mathbf{Z}$, the object $\operatorname{IC}_{\mu}(n)$ is $L^{+} \mathbf{G}_{m}{ }^{-}$ equivariant, and defines an object

$$
\operatorname{IC}_{\mu}(n) \in \operatorname{DM}\left(L^{+} \mathbf{G}_{m, S} \backslash\left(L^{+} G \backslash L G / L^{+} G\right)\right),
$$

supported on the Schubert variety $\mathrm{Gr}^{\leqslant \mu}$.

Proof. The statement about the support follows from Theorem 5.3.4(iii), and it is enough to prove that each $\mathrm{IC}_{\mu}(n)$ is $L^{+} \mathbf{G}_{m, s^{-}}$equivariant. But this is immediate from the $L^{+} \mathbf{G}_{m, S}$-invariance of the $L^{+} G$-orbits $\mathrm{Gr}^{\mu} \subset \mathrm{Gr}$ : by Proposition 3.2.20, 
we have $\operatorname{IC}_{\mu}(n) \in \mathrm{MTM}_{L^{+} G \rtimes L^{+} \mathbf{G}_{m, S}}(\mathrm{Gr})$. The latter category is a full subcategory of $\operatorname{DM}\left(L^{+} \mathbf{G}_{m, S} \backslash\left(L^{+} G \backslash L G / L^{+} G\right)\right)$ using Proposition 2.2.25 for the étale sheafifications.

\section{Intersection motives on moduli stacks of shtukas}

In this final section, we show that the intersection (cohomology) motive of the moduli stack of $G$-shtukas with bounded modification is defined independently of the standard conjectures on $t$-structures on triangulated categories of motives, cf. Corollary 6.3.5 below. Our presentation is expository in parts, and follows [Laf18, Section 2]. We put a stronger emphasis on the stack of relative positions, and the invariant which is the global function field analogue of the Grothendieck-Messing period map, cf. [SW13].

Let $X$ be a geometrically connected smooth projective curve over the finite field $k=\mathbf{F}_{q}$, and let $G$ be a split reductive $k$-group scheme.

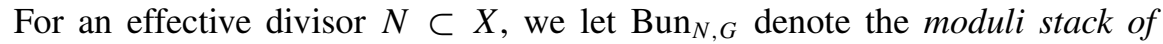
$G$-torsors on $X$ with level- $N$-structure viewed as an étale sheaf of groupoids $\left(\text { AffSch }_{k}\right)^{\text {op }} \rightarrow$ Gpd. Then $\operatorname{Bun}_{G}:=\operatorname{Bun}_{\varnothing, G}$ is representable by a quasiseparated Artin stack locally of finite type over $k$ (cf. for example [Hei10, Proposition 1]), and the forgetful map $\operatorname{Bun}_{N, G} \rightarrow \operatorname{Bun}_{G}$ is representable by a torsor under the restriction of scalars $\operatorname{Res}_{N / k}(G \times N)$ (a schematic smooth affine surjective map).

6.1. The stack of relative positions. We need the 'fusion version' of the loop group $L_{I} G \rightarrow X^{I}$ introduced in [BD99]. The relation to the loop group $L G \rightarrow S$ from Section 4 is explained in Example 6.1.3.

For a test scheme $T \in \mathrm{AffSch}_{k}$, and a relative effective Cartier divisor $D \subset$ $X_{T}$ which is finite and locally free over $T$, we denote by $\hat{D}_{T}$ the spectrum of the ring of global functions on the formal affine scheme $\left(X_{T} / D_{T}\right)^{\wedge}$ obtained as the completion of $X_{T}$ along $D_{T}$. Then $D_{T} \subset \hat{D}_{T}$ defines a Cartier divisor, and thus $\hat{D}_{T}^{o}:=\hat{D}_{T} \backslash D_{T}$ is an affine $k$-scheme as well, cf. [HR18b, Section 3.1.1] for details. For example, if $D$ is the graph of a point $x \in X(T)$, then $\hat{D}_{T}=$ $\operatorname{Spec}\left(R \llbracket \varpi_{x} \rrbracket\right)$ and $\hat{D}_{T}^{o}=\operatorname{Spec}\left(R\left(\left(\varpi_{x}\right)\right)\right)$ where $T=\operatorname{Spec}(R)$ and $\varpi_{x}$ is a local coordinate at $x \in X(T)$.

For any finite index set $I$, we consider the loop group functor $L_{I} G$ : $\left(\mathrm{AffSch}_{k}\right)^{\mathrm{op}} \rightarrow$ Sets defined by

$$
L_{I} G(T) \stackrel{\text { def }}{=}\left\{(x, g) \mid x=\left\{x_{i}\right\} \in X^{I}(T), g \in G\left(\hat{\Gamma}_{x}^{o}\right)\right\},
$$

where $\Gamma_{x} \subset X_{T}$ denotes the relative effective Cartier divisor given by the union of the graphs of the points $x_{i} \in X(T), i \in I$. Likewise, the positive loop 
group functor $L_{I}^{+} G:\left(\mathrm{AffSc}_{k}\right)^{\text {op }} \rightarrow$ Sets is defined as in (6.1.1) by replacing $\hat{\Gamma}_{x}^{o}$ with $\hat{\Gamma}_{x}$. The projection $L_{I} G \rightarrow X^{I}$ (respectively $L_{I}^{+} G \rightarrow X^{I}$ ) makes $L_{I} G$ (respectively $L_{I}^{+} G$ ) into an ind-affine $X^{I}$-group ind-scheme (respectively proalgebraic $X^{I}$-group scheme), cf. [HR18b, Lemma 3.2]. Note that $L_{I}^{+} G$ is a special case of the general set-up introduced in Proposition A.9 below by viewing $X^{I}$ as base scheme, and considering the relative curve $X^{I} \times X \rightarrow X^{I}$ together with the universal degree \#I divisor in $X^{I} \times X$, cf. also Example A.12(ii). Clearly, $L_{I}^{+} G \subset L_{I} G$ defines a subgroup functor over $X^{I}$.

DEFINITION 6.1.2. For any finite index set $I$, the étale sheaf of groupoids $\operatorname{AffSch}_{k}^{\text {op }} \rightarrow$ Gpd given by

$$
\operatorname{Pos}_{I} \stackrel{\text { def }}{=}\left(L_{I}^{+} G \backslash L_{I} G / L_{I} G^{+}\right)^{\text {ét }}
$$

is called the stack of relative positions.

This is an affine analogue of the relative position defined in Deligne-Lusztig theory, cf. [DL76, Section 1.2]. The importance of this stack lies in its relation to the Hecke stack (respectively moduli stack of shtukas) via the relative position (6.2.1) (respectively (6.3.2)).

Note that $\operatorname{Pos}_{I}$ is an fpqc sheaf of groupoids: this follows as in Lemma 5.3.2(i) using that every $L_{I}^{+} G$-torsor is étale-locally trivial by Proposition A.9.

EXAMPLE 6.1.3. Let $x: S \rightarrow X$ be a map where $S \in \mathrm{AffSch}_{k}$ is the spectrum of a local ring. Then, for $I=\{*\}$ a singleton, the fiber of $L_{I} G \rightarrow X$ (respectively $L_{I} G \rightarrow X$ ) over $x$ is the loop group $L G_{x}$ (respectively $L^{+} G_{x}$ ) considered in Section 4 formed by using as base scheme $S$, the group scheme $G_{x}:=G \times S$ and the local coordinate $\varpi=\varpi_{x}$ defined by $x$. Thus,

$$
\operatorname{Pos}_{I} \times_{X, x} S=\left(L^{+} G_{x} \backslash L G_{x} / L^{+} G_{x}\right)^{\text {ét }} .
$$

If $S$ is the spectrum of a field, the underlying topological space of $\left(L^{+} G_{x} \backslash L G_{x} / L^{+} G_{x}\right)^{\text {ét }}$ is the topological space associated with the partial ordered set $\left(X_{*}(T)_{+}, \leqslant\right)$, cf. Lemma 4.3.7 and Example 4.3.14. For $\lambda, \mu \in X_{*}(T)_{+}$, this means that $\mu$ specializes to $\lambda$ if and only if $\lambda \leqslant \mu$. For general $I$, the underlying topological space of $\operatorname{Pos}_{I}$ is a fusion version of the topological space $\left(X_{*}(T)_{+}, \leqslant\right)$with fusion structure induced by the monoid structure of $X_{*}(T)_{+}$, cf. [BD99, Section 5.3.10].

The following lemma is a slight reformulation of [MV07, Remark 5.1]. 
LEMMA 6.1.5. For $I=\{*\}$ a singleton, there is canonical map of étale sheaves of groupoids

$$
\operatorname{Pos}_{I} \rightarrow\left(L^{+} \mathbf{G}_{m} \backslash\left(L^{+} G \backslash L G / L^{+} G\right)\right)^{\text {ét }},
$$

where $L^{+} \mathbf{G}_{m}$ acts (as in Corollary 5.3.6) by changing the formal variable $\varpi$ used to form $L G, L^{+} G$.

6.2. The invariant. For any finite index set $I$, the Hecke stack Hecke ${ }_{I}$ is the étale sheaf of groupoids $\left(\mathrm{AffSch}_{k}\right)^{\text {op }} \rightarrow$ Gpd given by $T \mapsto\left(\mathcal{E}, \mathcal{E}^{\prime},\left\{x_{i}\right\}_{i \in I}, \alpha\right)$ where $\mathcal{E}, \mathcal{E}^{\prime} \in \operatorname{Bun}_{G}(T)$ are torsors, $\left\{x_{i}\right\}_{i \in I} \in X^{I}(T)$ are points, and $\alpha:\left.\mathcal{E}\right|_{X_{T} \backslash \cup x_{i}}$ $\left.\rightarrow \mathcal{E}^{\prime}\right|_{X_{T} \backslash \cup x_{i}}$ is a map (that is, an isomorphism) of torsors. A convenient notation (cf. [Hei18]) for the Hecke stack is

$$
\operatorname{Hecke}_{I}=\langle\mathcal{E} \underset{I}{\stackrel{\alpha}{\rightarrow}} \underset{\mathcal{E}}{\prime}\rangle,
$$

where $\alpha: \mathcal{E} \rightarrow \mathcal{E}^{\prime}$ is a birational map defined outside $\cup x_{i}$, that is, the torsor $\mathcal{E}^{\prime}$ differs from $\mathcal{E}$ by an 'algebraic modification' at a neighborhood of $\cup x_{i}$. The points $\left\{x_{i}\right\}_{i \in I}$ are called the paws (or legs) of the modification $\mathcal{E} \rightarrow-\mathcal{E}^{\prime}$. Further, Hecke $_{I}$ is representable by a quasiseparated ind-Artin stack, ind-(locally of finite type) over $k$ (cf. [Var04, Lemma 3.1]), and equipped with a forgetful map Hecke $_{I} \rightarrow X^{I}$.

Following the notation in [KR03] (cf. also [Zhu17, Section 1.2.1]), the relative position (or invariant)

$$
\text { inv: } \text { Hecke }_{I} \rightarrow \operatorname{Pos}_{I}, \quad\left(\mathcal{E} \stackrel{-\alpha}{I} \rightarrow \mathcal{E}^{\prime}\right) \mapsto \operatorname{inv}(\alpha),
$$

is the map of étale sheaves of groupoids over $X^{I}$ defined in terms of Proposition 2.2.25 as follows. For $T \in \operatorname{AffSch}_{X^{I}}$, and $\left(\mathcal{E} \rightarrow \mathcal{E}^{\prime}\right) \in \operatorname{Hecke}_{I}(T)$, we consider the étale sheaf $P:\left(\mathrm{AffSch}_{T}\right)^{\text {op }} \rightarrow$ Sets given by

$$
P\left(T^{\prime}\right)=\operatorname{Isom}\left(\left.\mathcal{E}^{\prime}\right|_{\hat{\Gamma}_{x}},\left.\mathcal{E}^{0}\right|_{\hat{\Gamma}_{x}}\right) \times \operatorname{Isom}\left(\left.\mathcal{E}\right|_{\hat{\Gamma}_{x}},\left.\mathcal{E}^{0}\right|_{\hat{\Gamma}_{x}}\right),
$$

where $x=\left\{x_{i}\right\}_{i \in I} \in X^{I}(T)$ are the legs of the modification, and $\mathcal{E}^{0}$ denotes the trivial torsor. The map $a: P \rightarrow T$ has the structure of a left $\left(L_{I}^{+} G \times L_{I}^{+} G\right) \times{ }_{X^{I}} T$ torsor via the rule $\left(g_{1}, g_{2}\right) *\left(\beta_{1}, \beta_{2}\right)=\left(g_{1} \beta_{1}, g_{2} \beta_{2}\right)$. It is étale-locally trivial by the approximation argument given in [HR18b, Lemma 3.4(ii)], and thus an étale torsor. We now define a map $b: P \rightarrow L_{I} G$ by sending $\left(\beta_{1}, \beta_{2}\right) \in P\left(T^{\prime}\right)$ to the element $\beta_{1} \alpha \beta_{2}^{-1} \in \operatorname{Aut}\left(\mathcal{E}_{0}\right)\left(\hat{\Gamma}_{x}^{o}\right)=G\left(\hat{\Gamma}_{x}^{o}\right)$. The map $b$ is equivariant for the left $L_{I}^{+} G \times L_{I}^{+} G$-action on $L_{I} G$ given by $\left(g_{1}, g_{2}\right) * g:=g_{1} g g_{2}^{-1}$. This defines the relative position

$$
\operatorname{inv}(\alpha):=\left(P \stackrel{b \times a}{\longrightarrow} L_{I} G \times T\right) \in\left(L_{I}^{+} G \backslash L_{I} G / L_{I} G^{+}\right)^{\text {ét }}(T)=\operatorname{Pos}_{I}(T) .
$$


DEFINITION 6.2.2 [Laf18, Definition 1.2]. For any effective divisor $N \subset X$, and any partition $I=I_{1} \sqcup \cdots \sqcup I_{r}, r \in \mathbf{Z}_{\geqslant 0}$, the iterated Hecke stack Hecke $\mathrm{I}_{N, I}^{\left(I_{1}, \ldots, I_{r}\right)}$ with level- $N$-structure is the étale sheaf of groupoids $\mathrm{AffSch}_{k} \rightarrow$ Gpd given by

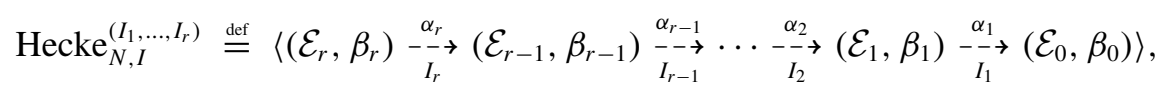

that is, Hecke $\operatorname{Hin}_{N, I}^{\left(I_{1}, \ldots, I_{r}\right)}(T)$ classifies data $\left(\left(\mathcal{E}_{j}, \beta_{j}\right)_{j=1, \ldots, r},\left\{x_{i}\right\}_{i \in I},\left(\alpha_{j}\right)_{j=1, \ldots, r}\right)$ where $\left(\mathcal{E}_{j}, \beta_{j}\right) \in \operatorname{Bun}_{N, G}(T)$ are torsors with level- $N$-structure, $\left\{x_{i}\right\}_{i \in I} \in(X \backslash N)^{I}(T)$ are points, and

$$
\alpha_{j}:\left.\left.\left(\mathcal{E}_{j}, \beta_{j}\right)\right|_{X_{T} \backslash\left(\cup_{i \in I_{j}} x_{i}\right)} \rightarrow\left(\mathcal{E}_{j-1}, \beta_{j-1}\right)\right|_{X_{T} \backslash\left(\cup_{i \in I_{j}} x_{i}\right)}
$$

are maps of torsors with level- $N$-structure.

As above Hecke ${ }_{N, I}^{\left(I_{1}, \ldots, I_{r}\right)}$ is representable by a quasiseparated ind-Artin stack indlocally of finite type over $k$, and equipped with the forgetful map Hecke $\operatorname{Hon}_{N, I}^{\left(I_{1}, \ldots, I_{r}\right)} \rightarrow$ $(X \backslash N)^{I} \subset X^{I}$. We need the following construction (cf. also [Laf18, (1.5)]): Fix a total order $I=\{1, \ldots, n\}, n=\# I$ compatible with the partition $I=I_{1} \sqcup \cdots \sqcup I_{r}$. This defines a refinement $I_{1}=\{1\} \sqcup \cdots \sqcup\left\{l_{1}\right\}, I_{2}=\left\{l_{1}+1\right\} \sqcup \cdots \sqcup\left\{l_{2}\right\}, \ldots$ and also the new partition $I=\{1\} \sqcup \cdots \sqcup\{n\}$. There are maps of étale sheaves of groupoids over $X^{I}$ given by

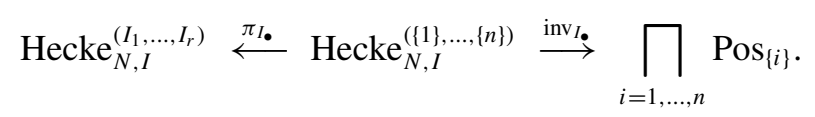

Here $\pi_{I_{0}}$ is given by forgetting certain $\left(\mathcal{E}_{j}, \beta_{j}\right)$ 's and composing the $\alpha_{j}$ 's in between as follows

$$
\begin{aligned}
& \left(\left(\mathcal{E}_{n}, \beta_{n}\right) \underset{\{n\}}{\stackrel{\alpha_{n}}{-\rightarrow}} \cdots \underset{\{1\}}{\stackrel{\alpha_{1}}{\rightarrow-\rightarrow}}\left(\mathcal{E}_{0}, \beta_{0}\right)\right)
\end{aligned}
$$

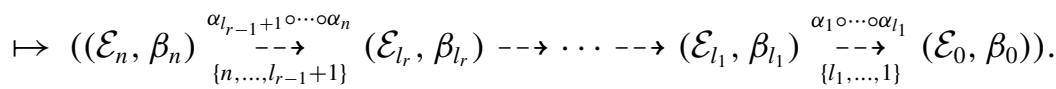

The relative position is given by $\operatorname{inv}_{I_{\bullet}}:\left(\left(\mathcal{E}_{\bullet}, \beta_{\bullet}\right), \alpha_{\bullet}\right) \mapsto\left(\operatorname{inv}\left(\alpha_{i}\right)\right)_{i=1, \ldots, n}$.

EXAMPLE 6.2.4. For $N=\varnothing$, and $I=\{1,2\}$ two elements $(r=1)$, the maps are given by

$$
\left(\mathcal{E}_{2} \underset{\{2,1\}}{\stackrel{\alpha_{1} \circ \alpha_{2}}{\rightarrow}} \mathcal{E}_{0}\right) \stackrel{\pi_{I_{\bullet}}}{\leftrightarrow}\left(\mathcal{E}_{2} \underset{\{2\}}{\stackrel{\alpha_{2}}{\longrightarrow}} \mathcal{E}_{1} \underset{\{1\}}{\stackrel{\alpha_{1}}{\rightarrow}} \mathcal{E}_{0}\right) \stackrel{\operatorname{inv}_{I_{\bullet}}}{\mapsto}\left(\operatorname{inv}\left(\alpha_{1}\right), \operatorname{inv}\left(\alpha_{2}\right)\right) .
$$


6.3. Intersection motives on moduli stacks of shtukas. The Hecke stack is used to construct the moduli stack of shtukas as follows, cf. [Laf18, Définition 2.1]. For any effective divisor $N \subset X$, and any partition $I=I_{1} \sqcup \cdots \sqcup I_{r}$, $r \in \mathbf{Z}_{\geqslant 0}$, the moduli stack of iterated $G$-shtukas $\operatorname{Sht}_{N, I}^{\left(I_{1}, \ldots, I_{r}\right)}$ with level- $N$ structure (or simply moduli stack of G-shtukas) is the étale sheaf of groupoids $\operatorname{AffSch}_{k}^{\text {op }} \rightarrow$ Gpd given by

$$
\left\langle\left(\mathcal{E}_{r}, \beta_{r}\right) \underset{I_{r}}{\stackrel{\alpha_{r}}{\rightarrow}}\left(\mathcal{E}_{r-1}, \beta_{r-1}\right) \underset{I_{r-1}}{\stackrel{\alpha_{r}}{-\rightarrow}} \cdots \underset{I_{2}}{\stackrel{\alpha_{2}}{\rightarrow}}\left(\mathcal{E}_{1}, \beta_{1}\right) \underset{I_{1}}{\stackrel{\alpha_{1}}{\rightarrow}}\left(\mathcal{E}_{0}, \beta_{0}\right)=\left({ }^{\tau} \mathcal{E}_{r},{ }^{\tau} \beta_{r}\right)\right\rangle,
$$

where ${ }^{\tau} \mathcal{E}:=\left(\operatorname{id}_{X} \times \text { Frob }_{T / k}\right)^{*} \mathcal{E}$ denotes the pullback, and Frob $_{T / k}$ is the relative Frobenius. Formally, $\operatorname{Sht}_{N, I}^{\left(I_{1}, \ldots, I_{r}\right)}$ is the fiber product of the forgetful map $\operatorname{Hecke}_{N, I}^{\left(I_{1}, \ldots, I_{r}\right)} \rightarrow \operatorname{Bun}_{G, N} \times \operatorname{Bun}_{G, N},\left(\left(\mathcal{E}_{r}, \beta_{r}\right) \rightarrow \cdots-\rightarrow\left(\mathcal{E}_{0}, \beta_{0}\right)\right) \mapsto$ $\left(\left(\mathcal{E}_{r}, \beta_{r}\right),\left(\mathcal{E}_{0}, \beta_{0}\right)\right)$ with the Frobenius correspondence id $\times$ Frob: $\operatorname{Bun}_{G, N} \rightarrow$ $\operatorname{Bun}_{G, N} \times \operatorname{Bun}_{G, N}$. There is the forgetful map $\operatorname{Sht}_{N, I}^{\left(I_{1}, \ldots, I_{r}\right)} \rightarrow(X \backslash N)^{I} \subset X^{I}$.

We fix a Borel pair $T \subset B \subset G$. By [Var04, Proposition 2.16] (cf. also [Laf18, Proposition 2.6]), there is a presentation of the reduced locus

$$
\left(\operatorname{Sht}_{N, I}^{\left(I_{1}, \ldots, I_{r}\right)}\right)_{\mathrm{red}}=\operatorname{colim}_{\underline{\mu}} \operatorname{Sht}_{N, I, \underline{\mu}}^{\left(I_{1}, \ldots, I_{r}\right)},
$$

with transition maps closed immersions. Here $\mu=\left(\mu_{i}\right)_{i \in I} \in X_{*}(T)_{+}$runs through the admissible tuples (that is, $\sum_{i \in I} \mu_{i}=0$ in $\pi_{1}(G)$ ), and each $\operatorname{Sht}_{N, I, \mu}^{\left(I_{1}, \ldots, I_{r}\right)}$ is representable by a nonempty Deligne-Mumford stack locally of finite type. Thus, (6.3.1) is an ind-Deligne-Mumford stack ind-(locally of finite type) over $k$.

Fixing a total order on $I=\{1, \ldots, n\}$ compatible with $I=I_{1} \sqcup \cdots \sqcup I_{r}$, the diagram (6.2.3) restricts to the diagram

$$
\operatorname{Sht}_{N, I}^{\left(I_{1}, \ldots, I_{r}\right)} \stackrel{\pi_{I_{\bullet}}}{\longleftarrow} \operatorname{Sht}_{N, I}^{(\{1\}, \ldots,\{n\})} \stackrel{\mathrm{inv}_{l} \bullet}{\longrightarrow} \prod_{i=1, \ldots, n} \operatorname{Pos}_{\{i\}} .
$$

A special case of Proposition 2.3.3 is the following result.

PROPOSITION 6.3.3. There exists an adjunction of functors

$$
\pi_{I_{\bullet} !}: \operatorname{DM}\left(\operatorname{Sht}_{N, I}^{(\{1\}, \ldots,\{n\})}\right) \leftrightarrows \operatorname{DM}\left(\operatorname{Sht}_{N, I}^{\left(I_{1}, \ldots, I_{r}\right)}\right): \pi_{I_{\bullet}}^{!}
$$

For any $\mu \in X_{*}(T)_{+}, m \in \mathbf{Z}$, we denote by

$$
\mathrm{IC}_{\{*\}, \mu}(m) \in \operatorname{DM}\left(\operatorname{Pos}_{\{*\}}\right)
$$

the !-pullback of $\operatorname{IC}_{\mu}(m) \in \operatorname{DM}\left(L^{+} \mathbf{G}_{m, k} \backslash\left(L^{+} G \backslash L G / L^{+} G\right)\right)$ under (6.1.6), cf. Corollary 5.3.6. 
DEFINITION 6.3.4. Fix a total order $I=\{1, \ldots, n\}$, and a compatible partition $I=I_{1} \sqcup \cdots \sqcup I_{r}$. For each effective divisor $N \subset X$, each admissible tuple $\mu=$ $\left(\mu_{i}\right)_{i \in I} \in X_{*}(T)_{+}$and each $\underline{m}=\left(m_{i}\right)_{i \in I} \in \mathbf{Z}^{I}$, one defines

$$
\mathcal{F}_{\underline{\mu}, \underline{m}}=\mathcal{F}_{N, I, \underline{\mu}, \underline{m}}^{\left(I_{1}, \ldots, I_{r}\right)} \stackrel{\text { def }}{=} \pi_{I_{\bullet} ! !}\left(\operatorname{inv}_{I_{\bullet}}^{!}\left(\bigotimes_{i=1}^{n} \operatorname{IC}_{\{i\}, \mu_{i}}\left(m_{i}\right)\right)\right) \in \operatorname{DM}\left(\operatorname{Sht}_{N, I}^{\left(I_{1}, \ldots, I_{r}\right)}\right) .
$$

(see Proposition 2.4.4 for the box product, Remark 2.2.2(ii) for the pullback and Proposition 6.3.3 for the pushforward).

COROLlary 6.3.5. Let $\ell \in \mathbf{Z}$ be a prime number invertible on $k$. For each tuple of data as in Definition 6.3.4, the motive $\mathcal{F}_{\underline{\mu}, \underline{m}}$ is supported on $\mathrm{Sht}_{N, I, \underline{\mu}}^{\left(I_{1}, \ldots, I_{r}\right)}$, and its $\ell$-adic realization

$$
\rho_{\ell}\left(\mathcal{F}_{\underline{\mu}, \underline{m}}\right) \in \mathrm{D}_{\text {ét }}\left(\operatorname{Sht}_{N, I, \underline{\mu}}^{\left(I_{1}, \ldots, I_{r}\right)}, \mathbf{Q}_{\ell}\right)
$$

is (up to twist and the choice of a lattice in the adelic center) the intersection complex defined in [Laf18, Définition 2.14]. In particular, the motives $\mathcal{F}_{\mu, \underline{m}}$ are normalized such that the $*$-restrictions of $\rho_{\ell}\left(\mathcal{F}_{\underline{\mu}, \underline{m}}\right)$ along the fibers of the map $p: \operatorname{Sht}_{N, I}^{\left(I_{1}, \ldots, I_{r}\right)} \rightarrow(X \backslash N)^{I}$ are perverse. Further, the $\ell$-adic realization of the motive

$$
p_{!}\left(\mathcal{F}_{\underline{\mu}, \underline{m}}\right) \in \mathrm{DM}\left((X \backslash N)^{I}\right)
$$

is (up to the normalizations above, and the bound of the Harder-Narasimhan slopes) the intersection cohomology complex defined in [Laf18, Définition 4.1].

REMARK 6.3.6. In [Laf18, Définition 2.14], the intersection complexes are normalized to be pure of weight zero along the fibers of the structure map $p$. For this reason, a square root of the cardinality of the residue field in a finite extension of $\mathbf{Q}_{\ell}$ is fixed there in order to define half Tate twists. Since this is not possible in the motivic setting, we have to add the Tate twists in Definition 6.3.4 as an additional datum.

Proof of Corollary 6.3.5. We need to relate the $\ell$-adic realization of $\mathcal{F}_{\underline{\mu}, \underline{m}}$ to the intersection complex of $\operatorname{Sht}_{N, I, \underline{\mu}}^{\left(I_{1}, \ldots, I_{r}\right)}$, cf. [Laf18, Definition 2.14]. There is a Cartesian diagram

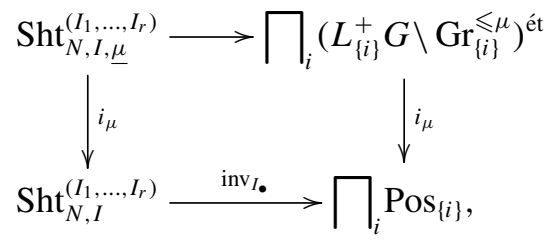


where $\operatorname{Gr}_{\{*\}}^{\leqslant \mu} \subset\left(L_{\{*\}} G / L_{\{*\}}^{+} G\right)^{\text {ét }}$ is the preimage of $\left(L^{+} \mathbf{G}_{m} \backslash \mathrm{Gr}_{G}^{\leqslant \mu}\right)^{\text {ét }}$ under (6.1.6). The $L_{\{*\}}^{+} G$-action on $\mathrm{Gr}_{\{*\}}^{\leqslant \mu}$ factors through a finite-dimensional quotient $L_{\{*\}}^{+} G \rightarrow$ $G_{j}, j \gg 0$, with split pro-unipotent kernel, cf. Proposition A.9. The top horizontal arrow induces the map onto the local model

$$
\epsilon: \operatorname{Sht}_{N, I, \underline{\mu}}^{\left(I_{1}, \ldots, I_{r}\right)} \rightarrow \prod_{i=1, \ldots, n}\left(G_{j} \backslash \mathrm{Gr}_{\{i\}}^{\leqslant \mu}\right)^{\text {ét }}
$$

constructed in [Laf18, Propositions 2.8, 2.9]. Denote $M:=\bigotimes_{i=1}^{n} \mathrm{IC}_{\{i\}, \mu_{i}}\left(m_{i}\right)$ which we view as a motive on the target of $\epsilon$ by Proposition 2.2.11. Together with Lemma 2.3.2 and base change for closed immersions (Proposition 2.3.3) it follows that there is an equivalence $\operatorname{inv}_{I_{0}}^{!} i_{\mu, !} M \simeq i_{\mu, !} \epsilon^{!} M$. Thus, [Laf18, Corollaries 2.16, 2.18] shows (the lattice in the adelic center does not affect the isomorphisms) that the $\ell$-adic realization of $\left(\pi_{I_{\bullet}} \circ i_{\mu}\right)_{!}\left(\epsilon^{!} M\right)$ is the intersection complex, cf. Proposition 6.3.3. Here we used Theorem 2.3.7 for the $\ell$-adic realization of the !-push forward. The rest of the corollary is immediate from this.

REMARK 6.3.7. (i) Similarly to [Laf18, Définition 4.1] one may also bound the Harder-Narasimhan slope of the bundles forming the shtuka in order to obtain locally constructible intersection cohomology motives.

(ii) There is an analogous version of Corollary 6.3 .5 for the fusion Grassmannians, cf. [Laf18, Theorem 1.17] for the $\ell$-adic version. We plan to improve on our result in two ways: independence of the intersection motives of the fixed total order on $I$ (and hence also in Corollary 6.3.5), and compatibility with the fusion structure coming from the motivic Satake equivalence [RS19]. Both statements rely on Whitney-Tate properties of fusion Grassmannians, and ultimately on the Tateness of the convolution morphism. This is work in progress.

(iii) Given (ii), it seems possible to obtain a $(S=T)$-Theorem in this context, cf. [Laf18, Proposition 6.2]. To proceed further, a major hurdle seems to be a variant of Drinfeld's lemma for DM.

\section{Appendix A. Ind-spaces and pro-groups}

In this appendix, we state our conventions about ind-algebraic spaces/indschemes (Appendix A.1), pro-algebraic groups (Appendix A.2) and their action on ind-algebraic spaces (Appendix A.3). In Appendix A.4, we prove that proalgebraic groups which are constructed as a 'positive loop group' (or 'jet group') satisfy a remarkable property: every torsor under such a pro-algebraic group admits sections étale-locally. 
A.1. Strict ind-spaces. Let $S$ be any scheme, and let $\mathrm{AffSch}_{S}$ be the category of affine schemes equipped with a map to $S$. A (strict) ind-algebraic space $X$ over $S$ is a presheaf $X:\left(\operatorname{AffSch}_{S}\right)^{\text {op }} \rightarrow$ Sets which admits a presentation $X=$ $\operatorname{colim}_{i} X_{i}$ where $\left\{X_{i}\right\}_{i \in I}$ is a direct system of algebraic spaces $X_{i}$ with transition maps $t_{i, j}: X_{i} \rightarrow X_{j}(j \geqslant i)$ being closed immersions. Here $I$ is a countable directed (a.k.a. filtered) index set. Every ind-algebraic space is an fpqc sheaf on $\mathrm{AffSch}_{S}$ (because every algebraic space defines a sheaf by [Sta17, Tag 03W8], and filtered colimits of sheaves on $\mathrm{AffSch}_{S}$ are computed termwise).

By definition, the category IndAlgSp $\mathrm{S}_{S}$ of ind-algebraic spaces over $S$ is a full subcategory of presheaves. Note that every map $T \rightarrow X$ from a quasicompact algebraic space factors over some $X_{i}$ (by quasicompactness of $T$ it is covered by finitely many affine schemes). Further, every map $f: \operatorname{colim}_{i \in I} X_{i} \rightarrow \operatorname{colim}_{j \in J} Y_{j}$ can be written as a colimit of maps $f_{(i, j)}: X_{(i, j)}:=X_{i} \times_{Y} Y_{j} \rightarrow Y_{j}=: Y_{(i, j)}$, $(i, j) \in I \times J$. In particular, after possibly changing the presentation every map $f: X \rightarrow Y$ is a colimit of maps $f_{i}: X_{i} \rightarrow Y_{i}$ for the same directed index set. Thus, the category IndAlgSp $\mathrm{p}_{S}$ is closed under fiber products, that is, $X \times_{Y} Z=\operatorname{colim}_{i} X_{i} \times_{Y_{i}} Z_{i}$ is an ind-algebraic space for any maps $X \rightarrow Y, Z \rightarrow Y$ in IndAlgSp ${ }_{S}$.

Let $\mathcal{P}$ be a property of algebraic spaces (or morphism of algebraic spaces). An ind-algebraic space $X$ (or a map $X \rightarrow Y$ ) is said to have ind- $\mathcal{P}$ if there exists a presentation $X=\operatorname{colim}_{i} X_{i}$ where each $X_{i}$ has property $\mathcal{P}$. A map $f: X \rightarrow Y$ of ind-algebraic spaces is said to have property $\mathcal{P}$ (respectively to be schematic and to have $\mathcal{P}$ ) if for all $T \in \operatorname{AffSch}_{S}$, the pullback $X \times_{Y} T$ is an algebraic space (respectively a scheme) and the map $f \times_{Y} T$ has property $\mathcal{P}$.

Likewise, the category $\operatorname{IndSch}_{S}$ of (strict) ind-schemes over $S$ is the full subcategory of $\operatorname{IndAlgSp} \mathrm{p}_{S}$ of those objects $X=\operatorname{colim}_{i} X_{i}$ where each $X_{i}$ is a scheme.

A.2. Strictly pro-algebraic groups. Let $S$ be any scheme. A (strictly) proalgebraic group scheme $G$ over $S$ is a presheaf $G:\left(\mathrm{AffSch}_{S}\right)^{\mathrm{op}} \rightarrow$ Grps which admits a presentation $G=\lim _{i} G_{i}$ where $\left\{G_{i}\right\}_{i \in \mathbf{N}}$ is an inverse system of smooth $S$-affine (hence finitely presented) $S$-group schemes $G_{i}$ with smooth surjective transition maps of $S$-groups $\pi_{i, j}: G_{j} \rightarrow G_{i}$ for $j \geqslant i$.

LEMMA A.1. Let $G=\lim _{i \in \mathbf{N}} G_{i}$ be a pro-algebraic $S$-group.

(i) The presheaf $G$ is representable by a faithfully flat $S$-affine $S$-group scheme.

(ii) For each $i \in \mathbf{N}$, the map $G \rightarrow G_{i}$ is faithfully flat, and hence there is a short exact sequence of flat $S$-affine $S$-group schemes $1 \rightarrow U_{i} \rightarrow G \rightarrow G_{i} \rightarrow 1$.

(iii) If all the $G_{i}$ have connected fibers over $S$, then so does $G$ in which case they are automatically geometrically connected. 
Proof. Let $\mathcal{A}_{i}$ be the Hopf $\mathcal{O}_{S}$-algebras defining $G_{i}$. Parts (i) and (ii) follow by noting that the colimit $\mathcal{A}:=\operatorname{colim}_{i} \mathcal{A}_{i}$ has a natural Hopf algebra structure. It is faithfully flat since all the $G_{j} \rightarrow G_{i}$ are smooth surjective. Part (i) and (ii) are immediate. For (iii), note that any (pro-)algebraic $S$-group $G$ with connected fibers automatically has geometrically connected fibers by [Sta17, Tag 04KV] (because the unit section always defines a rational point). Hence, we may assume that $S$ is the spectrum of an algebraically closed field, so that the topological space $\left|G_{i}\right|$ is connected for every $i \in \mathbf{N}$. Using that the map $|G| \rightarrow\left|G_{i}\right|, i \in \mathbf{N}$ is surjective and open (being quasicompact, surjective and flat [Sta17, Tag 02JY]), one checks that $|G|$ is connected.

A.3. Pro-algebraic groups acting on ind-algebraic spaces. Let $G$ be a proalgebraic group and $X$ an ind-algebraic space over $S$. Then a map of presheaves $a: G \times{ }_{S} X \rightarrow X$ which satisfies the axioms of an action map is called an action of $G$ on $X$ (over $S$ ).

Definition A.1. The action $a: G \times_{S} X \rightarrow X$ is called admissible if there exist presentations $G=\lim _{i \in \mathbf{N}} G_{i}$ and $X=\operatorname{colim}_{j \in J} X_{j}$ with the following properties:

(i) The presentation $X=\operatorname{colim}_{j} X_{j}$ is $G$-stable, that is, for each $j \in J$, the restriction $\left.a\right|_{G \times{ }_{S} X_{j}}$ factors as $G \times{ }_{S} X_{j} \stackrel{a_{j}}{\rightarrow} X_{j} \subset X$.

(ii) For each $j \in J$, the $G$-action on $X_{j}$ factors through the algebraic $S$-group $G_{i}$ for some $i \gg 0$, that is, the subgroup $U_{i}=\operatorname{ker}\left(G \rightarrow G_{i}\right)$ operates trivially on $X_{j}$.

LEMMA A.2. Let $a: G \times{ }_{S} X \rightarrow X$ be an admissible action for the presentations $G=\lim _{i \in \mathbf{N}} G_{i}, X=\operatorname{colim}_{j \in J} X_{j}$. By taking suitable finite unions of the $X_{j}$, there exists a $G$-stable presentation $X=\operatorname{colim}_{i \in \mathbf{N}} X_{i}^{\prime}$ such that the $G$-action on $X_{i}^{\prime}$ factors through $G_{i}$ for every $i \in \mathbf{N}$.

Proof. For each $i \in \mathbf{N}$, let $J_{i}=\left\{j \in J \mid\right.$ the $G$-action on $X_{j}$ factors exactly through $\left.G_{i}\right\}$. Then the sets $J_{i}$ are countable, pairwise disjoint, and one has $\bigsqcup_{i \in \mathbf{N}} J_{i}=J$ by Definition A.1(ii). Cantor's diagonal argument produces a family of finite subsets $\left\{J_{i}^{\prime}\right\}_{i \in \mathbf{N}}$ of $J$ with the following properties: for each $j \in J_{i}^{\prime}$ the $G$-action on $X_{j}$ factors through $G_{i}$, one has $J_{i}^{\prime} \subset J_{i^{\prime}}^{\prime}$ for $i \leqslant i^{\prime}$, and $\bigcup_{i \in \mathbf{N}} J_{i}^{\prime}=J$. For each $i \in \mathbf{N}$, we define the closed subspace $X_{i}^{\prime}:=\bigcup_{j \in J_{i}^{\prime}} X_{j} \subset X$, that is, the scheme-theoretic image (cf. [Sta17, Tag 082W]) of the quasicompact map $\bigsqcup_{j \in J_{i}^{\prime}} X_{j} \rightarrow X$. Note that we have a presentation $X=\operatorname{colim}_{i} X_{i}^{\prime}$. To prove that $X_{i}^{\prime}$ is $G$-stable, we note 
that the diagram of ind-algebraic spaces

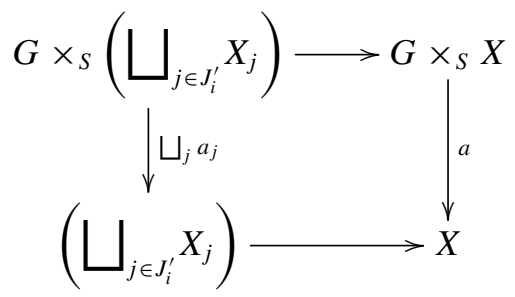

is Cartesian. Since $G$ is $S$-flat and taking the scheme-theoretic image along quasicompact maps commutes with flat base change (follows from [Sta17, Tag 082Z]), the scheme-theoretic image of the top arrow is $G \times{ }_{S} X_{i}^{\prime}$. This implies that $X_{i}^{\prime}$ is $G$-stable. By construction, the $G$-action on $X_{i}^{\prime}$ factors through $G_{i}$.

LEMmA A.3. Let $a: G \times{ }_{S} X \rightarrow X$ be an admissible action for the presentations $G=\lim _{i \in \mathbf{N}} G_{i}, X=\operatorname{colim}_{j \in J} X_{j}$. Then the action restricts to an action $a_{\mathrm{red}}: G \times{ }_{S} X_{\mathrm{red}} \rightarrow X_{\mathrm{red}}$ on the underlying reduced sub-ind-algebraic space which is admissible for the presentations $G=\lim _{i} G_{i}$ and $X_{\mathrm{red}}=\operatorname{colim}_{j} X_{j, \mathrm{red}}$.

Proof. Once we know that $X_{j, \text { red }} \subset X_{j}, j \in J$ is $G$-invariant, the admissibility of the induced action is immediate. We reduce to the case where $X=X_{j}$ is an algebraic space. We need to show that the reduced subspace $X_{\text {red }} \subset X$ is $G$-invariant. Our claim follows, by applying the functor $(-)_{\text {red }}$, from the following equality of algebraic spaces

$$
G \times{ }_{S} X_{\text {red }}=\left(G \times{ }_{S} X\right)_{\text {red }} .
$$

If $G$ is a smooth $S$-group, then (A.4) holds true because being reduced is local in the smooth topology, cf. [Sta17, Tag 034E]. The general case follows from $\mathcal{A}=$ $\operatorname{colim}_{i} \mathcal{A}_{i}$, in the notation of the proof of Lemma A.1, and the compatibility of tensor products and colimits using that $\mathcal{A}_{i} \rightarrow \mathcal{A}_{j}$ is universally injective (because faithfully flat).

LEMmA A.5. Let $X$ be of ind-finite type over a Noetherian scheme $S$, and let $G$ be a pro-algebraic $S$-group. Then every action $a: G \times{ }_{S} X \rightarrow X$ is admissible.

Proof. Let $X=\operatorname{colim}_{j \in J} X_{j}$ be a presentation by finite type $S$-algebraic spaces, and let $a: G \times{ }_{S} X \rightarrow X$ be an action. As $G$ is an $S$-affine scheme, the algebraic space $G \times{ }_{S} X_{j}, j \in J$ is quasicompact. Hence, the map $\left.a\right|_{G \times_{S} X_{j}}$ factors through $X_{j^{\prime}}$ for some $j^{\prime} \gg 0$, and we define $X_{j}^{\prime}$ as the scheme-theoretic image of $\left.a\right|_{G \times S} X_{j}$. Since $S$ is Noetherian, the closed subspace $X_{j}^{\prime} \subset X_{j^{\prime}}$ is of finite type over $S$, and 
clearly $G$-stable by construction. Also $X=\operatorname{colim}_{j} X_{j}^{\prime}$ because $X_{j} \subset X_{j}^{\prime}$. Now one verifies that every $G$-action on any finite type $S$-algebraic space $X$ factors through $G_{i}$ for some $i \gg 0$. The lemma follows.

A.4. Torsors under pro-algebraic groups. Let $G$ be a pro-algebraic $S$-group. By Lemma A.1, the map $G \rightarrow S$ is faithfully flat and affine (hence quasicompact). Let $P \rightarrow S$ be a right $G$-torsor in the fpqc topology on $S$. By fpqc descent for affine morphisms [Sta17, Tag 0245], the map $P \rightarrow S$ is also faithfully flat and affine (hence $P$ is a scheme). We denote the set of isomorphism classes of right $G$-torsors in the fpqc (respectively étale) topology on $S$ by $\mathrm{H}_{\mathrm{fpqc}}^{1}(S, G)$ (respectively $\mathrm{H}_{\mathrm{et}}^{1}(S, G)$ ). (Lemma A.3 below implies that $\mathrm{H}_{\mathrm{fpqc}}^{1}(S, G)$ is indeed a set by using the twisting trick.)

Our aim is to show that $\mathrm{H}_{\mathrm{fpqc}}^{1}(S, G)=\mathrm{H}_{\mathrm{ett}}^{1}(S, G)$ under suitable conditions on $G$ (cf. Corollary A.8), and to show that all examples we have in mind satisfy this condition, cf. Proposition A.9 and Example A.12. This generalizes the étale-local triviality of the torsors considered in [PR08, Theorem 1.4] and [HR18b, Lemma 3.4(ii)] for example.

Given a presentation $G=\lim _{i \in \mathbf{N}} G_{i}, P_{i}:=P \times{ }^{G} G_{i}$ is a $G_{i}$-torsor on $S$. For each $i \in \mathbf{N}$, the transition map $G_{i+1} \rightarrow G_{i}$ induces an identification $P_{i+1} \times{ }^{G_{i+1}}$ $G_{i}=P_{i}$. This gives a natural map

$$
\mathrm{H}_{\mathrm{fpqc}}^{1}(S, G) \rightarrow \lim \mathrm{H}_{\mathrm{et}}^{1}\left(S, G_{i}\right), \quad P \mapsto\left\{P_{i}\right\}_{i \in \mathbf{N}} .
$$

Here we use that $\mathrm{H}_{\mathrm{et}}^{1}\left(S, G_{i}\right) \subset \mathrm{H}_{\mathrm{fpqc}}^{1}\left(S, G_{i}\right)$ is a bijection: as $G_{i} \rightarrow S$ is smooth, any fpqc- $G_{i}$-torsor is also smooth, and hence admits sections étale-locally. As in [BK72, Ch. IX, Section 2] consider the group $\prod_{i \in \mathbf{N}} \mathrm{H}^{0}\left(S, G_{i}\right)$ acting on the set $\prod_{i \in \mathbf{N}} \mathrm{H}^{0}\left(S, G_{i}\right)$ via the formula

$$
\left(g_{0}, g_{1}, g_{2}, \ldots\right) *\left(x_{0}, x_{1}, x_{2}, \ldots\right):=\left(g_{0} x_{0} g_{1}^{-1}, g_{1} x_{1} g_{2}^{-1}, g_{2} x_{2} g_{3}^{-1}, \ldots\right),
$$

where $G_{i+1}$ acts on $G_{i}$ via the transition map $G_{i+1} \rightarrow G_{i}$. We denote by $\lim ^{1} \mathrm{H}^{0}\left(S, G_{i}\right):=\prod \mathrm{H}^{0}\left(S, G_{i}\right) / \sim$ its set of equivalence classes. There is a natural map

$$
\prod \mathrm{H}^{0}\left(S, G_{i}\right) \rightarrow \mathrm{H}_{\mathrm{fpqc}}^{1}(S, G), \quad\left(x_{i}\right)_{i \in \mathbf{N}} \mapsto \lim _{x_{i}} G_{i},
$$

where $G_{i}$ is considered as the trivial $G_{i}$-torsor via right multiplication with transition maps $G_{i+1} \rightarrow G_{i}, a \mapsto x_{i} \cdot a$. Then $\left\{G_{i}, x_{i}\right\}_{i \in \mathbf{N}}$ forms an inverse system,

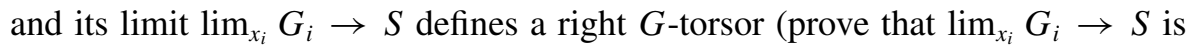
faithfully flat and affine similarly to the proof of Lemma A.1, and further that the right $G$-action is simply transitive). 
LEMmA A.3. The maps (A.1) and (A.2) induce an exact sequence of pointed sets

$$
1 \rightarrow \lim ^{1} \mathrm{H}^{0}\left(S, G_{i}\right) \rightarrow \mathrm{H}_{\mathrm{fpqc}}^{1}(S, G) \rightarrow \lim _{\mathrm{H}_{\mathrm{et}}}^{1}\left(S, G_{i}\right) \rightarrow 1 .
$$

Proof. The map $\lim ^{1} \mathrm{H}^{0}\left(S, G_{i}\right) \rightarrow \mathrm{H}^{1}(S, G)$ is well-defined. Indeed, if $x, y \in$ $\prod \mathrm{H}^{0}\left(S, G_{i}\right)$ with $y=g * x$ for some $g \in \prod \mathrm{H}^{0}\left(S, G_{i}\right)$, then there is an isomorphism of inverse systems $\left\{G_{i}, x_{i}\right\}_{i \in \mathbf{N}} \rightarrow\left\{G_{i}, y_{i}\right\}_{i \in \mathbf{N}}$ induced from the maps $G_{i} \rightarrow G_{i}, a \mapsto g_{i} \cdot a$. The exactness properties are elementary to check, and left to the reader. Note that this can also be regarded as an example of a Milnor type exact sequence [GJ09, Proposition VI.2.15].

Let $\mathcal{E}$ be a $S$-vector bundle, that is, a locally free $\mathcal{O}_{S}$-module of finite rank. Using that $\mathcal{E}$ is quasicoherent and reflexive (that is, $\left.\mathcal{E}=\left(\mathcal{E}^{\vee}\right)^{\vee}\right)$, one shows that the group-valued functor on the category of $S$-schemes $T$ given by $T \mapsto \mathcal{E}(T)$ is representable by the $S$-group scheme

$$
\mathbb{V}(\mathcal{E}):=\underline{\operatorname{Spec}}_{\mathcal{O}_{S}}\left(\operatorname{Sym}^{\otimes}\left(\mathcal{E}^{\vee}\right)\right) \rightarrow S,
$$

cf. [Gro61, Section 1.7]. Note that our notion is dual to the reference. We also write $\mathbb{V}^{\times}(\mathcal{E}):=\mathbb{V}(\mathcal{E}) \backslash S$ for the complement of the zero section. The $S$-group $\mathbb{V}(\mathcal{E})$ is algebraic, unipotent, commutative, and Zariski-locally on $S$ isomorphic to $\mathbf{G}_{a, S}^{r}, r:=\operatorname{rank}(\mathcal{E})$. A vector group is an $S$-group isomorphic to $\mathbb{V}(\mathcal{E})$ for some $S$-vector bundle $\mathcal{E}$.

DEFINITION A.5. A pro-algebraic $S$-group $G$ is called split pro-unipotent if it admits a presentation $G=\lim _{i \in \mathbf{N}} G_{i}$ such that the group $G_{0}$ and all groups $\operatorname{ker}\left(G_{i+1} \rightarrow G_{i}\right), i \in \mathbf{N}$ are vector groups. In other words, a split pro-unipotent group is an (possibly infinite) successive extension of a vector group by vector groups.

Proposition A.6. If $S$ is affine and $G$ is split pro-unipotent, then $\mathrm{H}_{\mathrm{fpqc}}^{1}(S, G)$ is trivial.

Proof. We have $\mathrm{H}_{\text {ét }}^{1}\left(S, G_{i}\right)=1$ for all $i$. Indeed, by induction on $i$ and the standard 6-term exact sequence for non-abelian group cohomology [Gir71, Ch. III, Proposition 3.3.1], this reduces to $\mathrm{H}_{\text {ét }}^{1}(S, \mathbb{V}(\mathcal{E}))=\mathrm{H}_{\text {ét }}^{1}(S, \mathcal{E})=\mathrm{H}_{\text {Zar }}^{1}(S, \mathcal{E})$ [Sta17, Tag 03P2] being trivial which holds because $S$ is affine. This argument also shows that the maps $\mathrm{H}^{0}\left(S, G_{i+1}\right) \rightarrow \mathrm{H}^{0}\left(S, G_{i}\right)$ are surjective, so $\lim ^{1} \mathrm{H}^{0}$ $\left(S, G_{i}\right)=1$ by [BK72, Ch. IX, Section 2, Proposition 2.4]. Thus, the proposition follows from Lemma A.3. 
EXAMPLE A.7. Proposition A.6 is false for general pro-algebraic groups: Let $p \in \mathbf{Z}$ be a prime, and let $G=\operatorname{Gal}\left(\mathbf{Q}_{p}\left(\zeta_{p^{\infty}}\right) / \mathbf{Q}_{p}\right) \simeq \mathbf{Z}_{p}$ considered as a proalgebraic group. Then the $G$-torsor $\operatorname{Spec}\left(\mathbf{Q}_{p}\left(\zeta_{p^{\infty}}\right)\right) \rightarrow \operatorname{Spec}\left(\mathbf{Q}_{p}\right)$ has no sections étale-locally.

COROLlary A.8. If there exists an $i_{0} \in \mathbf{N}$ such that $\operatorname{ker}\left(G \rightarrow G_{i_{0}}\right)$ is split prounipotent, then the natural map $\mathrm{H}_{\mathrm{et}}^{1}(S, G) \rightarrow \mathrm{H}_{\mathrm{fpqc}}^{1}(S, G)$ is bijective.

Proof. The map is clearly injective, and we have to show that every $G$-torsor $P \rightarrow$ $S$ for the fpqc topology admits sections étale-locally. For this we may assume $S$ is affine. Let $U:=\operatorname{ker}\left(G \rightarrow G_{i_{0}}\right)$, and consider the factorization $P \stackrel{a}{\rightarrow} P / U=$ $P \times{ }^{G} G_{i_{0}} \stackrel{b}{\rightarrow} S$. The map $b$ is a $G_{i_{0}}$-torsor, and hence admits sections étale-locally. The map $a$ is a trivial $U$-torsor by Proposition A.6, since $S$ and therefore $P / U$ is affine as well.

We end this section by proving that pro-algebraic groups which are defined as positive loop groups (or sometimes called jet groups) satisfy the assumption of Corollary A. 8 with $i_{0}=0$. All examples of these pro-algebraic groups which we encounter in the main body of this manuscript fall under the following general set-up, cf. Example A.12 below.

Let $X \rightarrow S$ be smooth and pure of relative dimension 1. Let $D \subset X$ be an effective Cartier divisor which is finite and locally free over $S$. Let $\mathcal{I}_{D} \subset \mathcal{O}_{X}$ be the ideal sheaf defined by $D$. For $i \geqslant 0$, the subscheme $D_{i} \subset X$ defined by $\mathcal{I}_{D}^{i+1}$ is again finite and locally free over $S$. Let $\hat{D}=\operatorname{colim}_{i \in \mathbf{N}} D_{i}$ considered as an ind-scheme. Let $\hat{\mathcal{G}} \rightarrow \hat{D}$ be a group functor which is relatively representable by a smooth affine group scheme of finite presentation. For each $i \geqslant 0$, let $\mathcal{G}_{i}:=$ $\hat{\mathcal{G}} \times{ }_{\hat{D}} D_{i}$. (We do not want to require that $\hat{\mathcal{G}}$ spreads to a group scheme over $X$. The weaker assumption suffices for our purposes.) We consider the strictly proalgebraic $S$-group (cf. proof of Proposition A.9)

$$
G \stackrel{\text { def }}{=} \lim _{i \geqslant 0} G_{i}
$$

where $G_{i}:=\operatorname{Res}_{D_{i} / S}\left(\mathcal{G}_{i}\right)$ denotes the Weil restriction of scalars, and $G_{i} \rightarrow G_{i-1}$ are the obvious transition maps. Further, we denote $U:=\operatorname{ker}\left(G \rightarrow G_{0}\right)$.

Proposition A.9. The pro-algebraic group $U$ is split pro-unipotent. More precisely, for each $i \geqslant 1$, there is a short exact sequence of algebraic $S$-group schemes

$$
0 \rightarrow \mathbb{V}\left(\mathcal{E}_{i}\right) \rightarrow G_{i} \rightarrow G_{i-1} \rightarrow 0
$$


for an explicit $S$-vector bundle $\mathcal{E}_{i}$ of $\operatorname{rank} \operatorname{rk}(D) \cdot \operatorname{dim}(\hat{\mathcal{G}} / \hat{D})$, viewed as a locally constant function on $S$. In particular, by Corollary A.8, we have $\mathrm{H}_{\mathrm{fpqc}}^{1}(S, G)=$ $\mathrm{H}_{\mathrm{et}}^{1}(S, G)$.

Proof. Since $D_{i} \rightarrow S$ is finite locally free, the Weil restriction of scalars $G_{i}$ is representable by an algebraic $S$-group scheme, cf. [BLR90, Section 7.6, Theorem 4, Proposition 5]. The canonical map $G_{i} \rightarrow G_{i-1}$ is locally of finite presentation (because limit preserving [Sta17, Tag 01ZC]), formally smooth, and thus is a smooth map, cf. [Sta17, Tag 02H6]. As $G_{i} \rightarrow G_{i-1}$ is also surjective, it follows that $G_{i} \rightarrow G_{i-1}$ is a surjection of étale sheaves. It remains to identify the kernel $\operatorname{ker}\left(G_{i} \rightarrow G_{i-1}\right)$ as a vector group.

We consider the following general set-up. Let $S^{\prime}$ be a base scheme, and let $Y, Z$ be $S^{\prime}$-schemes. Let $Y_{0} \subset Y$ be a closed subscheme defined by a sheaf of ideals $\mathcal{J}$ with $\mathcal{J}^{2}=0$. Let $g_{0}: Y_{0} \rightarrow Z$ be a map of $S^{\prime}$-schemes. If $Z \rightarrow S^{\prime}$ is smooth, then [SGA03, III.5, Corollary 5.2] implies that for all $T \rightarrow S^{\prime}$ we have a functorial identification

$$
\left\{g \in \operatorname{Hom}_{T}\left(Y_{T}, Z_{T}\right)|g|_{Y_{0, T}}=g_{0, T}\right\}=\left(g_{0}^{*} \mathfrak{g}_{Z / S} \otimes_{\mathcal{O}_{Y_{0}}} \mathcal{J}\right)\left(Y_{0, T}\right)
$$

where $\mathfrak{g}_{Z / S}:=\left(\Omega_{Z / S}^{1}\right)^{*}$. We apply this as follows.

Let $S^{\prime}=D_{i}$, and set $Z:=\mathcal{G}_{i}$. Let $Y_{0}:=D_{i-1} \subset D_{i}=: Y$, that is, $\mathcal{J}=\mathcal{I}_{D}^{i} / \mathcal{I}_{D}^{i+1}$. Let $g_{0}: D_{i-1} \rightarrow \mathcal{G}_{i}$ be given by the inclusion $D_{i-1} \subset D_{i}$ composed with the identity section 1: $D_{i} \rightarrow \mathcal{G}_{i}$. Also let $\pi_{i}: D_{i} \rightarrow S$ be structure map. Taking $\pi_{i, *}$ of the left hand side in (A.10), that is, restricting the functor to the category of $S$-schemes, is by definition equal to the functor $\operatorname{ker}\left(G_{i} \rightarrow G_{i-1}\right)$. We define

$$
\mathcal{E}_{i} \stackrel{\text { def }}{=} \pi_{i, *}\left(\operatorname{Lie}^{(i)}(\hat{\mathcal{G}}) \otimes_{\mathcal{O}_{D_{i-1}}}\left(\mathcal{I}_{D}^{i} / \mathcal{I}_{D}^{i+1}\right)\right),
$$

where $\operatorname{Lie}^{(i)}(\hat{\mathcal{G}}):=1^{*}\left(\Omega_{\mathcal{G}_{i-1} / D_{i-1}}^{1}\right)^{*}$. Note that $\operatorname{Lie}^{(i)}(\hat{\mathcal{G}})$ is a locally free $\mathcal{O}_{D_{i-1}}$ module of rank $\operatorname{dim}(\hat{\mathcal{G}} / \hat{D})$ because $\hat{\mathcal{G}} \rightarrow \hat{D}$ is smooth. Since $\mathcal{I}_{D}^{i} / \mathcal{I}_{D}^{i+1}$ is locally free of $\operatorname{rank} \operatorname{rk}(D)$ when considered as an $\mathcal{O}_{S}$-module, we see that $\mathcal{E}$ is a locally free $\mathcal{O}_{S}$-module of $\operatorname{rank} \operatorname{rk}(D) \cdot \operatorname{dim}(\hat{\mathcal{G}} / \hat{D})$. Further, the argument above shows that $\mathbb{V}\left(\mathcal{E}_{i}\right)=\operatorname{ker}\left(G_{i} \rightarrow G_{i-1}\right)$ as $S$-schemes, and a calculation using $\mathcal{J}^{2}=0$ shows that the identification is compatible with the group structure.

Here is a list of examples which are of interest to us.

EXAmple A.12. (i) Loop groups. Let $S=\operatorname{Spec}(k)$ for some field $k, X=\mathbf{A}_{k}^{1}$, and $D=\{0\}$. Then $\hat{D}=\operatorname{Spf}(k \llbracket \varpi \rrbracket)$ where $\varpi$ is a local parameter at 0 , and we let $\hat{\mathcal{G}}=H \times{ }_{S} \operatorname{Spf}(k \llbracket \varpi \rrbracket)$ for a smooth affine finite type $k$-group $H$. In this 
case, $G=L^{+} H$ which is the functor on the category of $k$-algebras $R$ given by $L^{+} H: R \mapsto H(R \llbracket \varpi \rrbracket)$.

(ii) Fusion loop groups. Let $Y$ be a smooth curve over the field $k$. For any finite index set $I$, let $S:=Y^{I}$, and $X:=S \times_{k} Y \rightarrow S$ the projection. Let $D \subset X$ be the universal degree \#I divisor. Let $H$ be a smooth affine finite type $k$-group, and let $\hat{\mathcal{G}}:=H \times_{k} \hat{D}$. Then $G=L_{I}^{+} H \rightarrow Y^{I}$ is the fusion loop group from (6.1.1) introduced in [BD99]. We make the case \#I=2 more explicit. Let $\Delta \subset Y^{2}$ be the diagonal with complement $U=Y^{2} \backslash \Delta$. Then $\left.L_{I}^{+} H\right|_{U}=\left(L^{+} H \times{ }_{k} L^{+} H\right) \times_{k} U$ whereas $\left.L_{I}^{+} H\right|_{\Delta}=L^{+} H \times_{k} \Delta$. We observe that the group $G_{0}=\operatorname{Res}_{D / Y^{2}}\left(H \times_{k} D\right)$ $\rightarrow Y^{2}$ is not reductive. Indeed, $\left.G_{0}\right|_{U}=H \times_{k} H$, but $\left.G_{0}\right|_{\Delta}=H \ltimes \operatorname{Lie}^{(1)}(H)$ because $D \rightarrow Y^{2}$ is ramified along $\Delta$ (for example, take $Y=\mathbf{A}_{k}^{1}$, then $D=\{f=0\}$ for $f=\left(\varpi-x_{1}\right)\left(\varpi-x_{2}\right)$ where $x_{1}, x_{2}$ are the coordinates on $S=\mathbf{A}_{k}^{2}$, and $\varpi$ is the coordinate on $\mathbf{A}_{k}^{1}$ in $\left.X=\mathbf{A}_{k}^{2} \times_{k} \mathbf{A}_{k}^{1}\right)$. This is in accordance with $\operatorname{ker}\left(G_{1} \rightarrow G_{0}\right)$ being a vector group.

(iii) Nonconstant group schemes. Let $S=\operatorname{Spec}(R)$ for some $\operatorname{ring} R$. Let $X=\mathbf{A}_{R}^{1}$, and let $D=\{f=0\}$ for some polynomial $f \in R[\varpi]$.

(iii(a)) Specialize to $f=\varpi$, and $\mathcal{G}$ a smooth affine $R \llbracket \varpi \rrbracket$-group scheme. Define $\hat{\mathcal{G}}:=\mathcal{G} \times{ }_{\operatorname{Spec}(R \llbracket \varpi \rrbracket)} \operatorname{Spf}(R \llbracket \varpi \rrbracket)$. In the case $R=k$ is a field, the group $G$ is the twisted positive loop group in the sense of [PR08].

(iii(b)) Specialize to $R=\mathbf{Z}_{p}$ for $p$ a prime number, $f=\varpi-p$ (respectively any Eisenstein polynomial). Let $\mathcal{G}$ be a smooth affine $\mathbf{A}_{\mathbf{z}_{p}}^{1}$-group scheme, for example, one of the group schemes constructed in Pappas and Zhu [PZ13, Section 4] (respectively Levin [Lev16, Section 3]). Then $G$ over $\mathbf{Z}_{p}$ is the positive loop group constructed in [PZ13, (6.4), 6.2.6] (respectively [Lev16, Proposition 4.1.4 ff]), cf. also [HR18b, Example 3.1(ii)].

\section{Acknowledgements}

The authors greatly benefited from discussions with Johannes Anschütz, DenisCharles Cisinski, Laurent Fargues, Dennis Gaitsgory, Jochen Heinloth, Simon Henry, Thomas Nikolaus, Peter Scholze, Benoît Stroh, Torsten Wedhorn and Xinwen Zhu around the subject of the manuscript, and it is a pleasure to thank all of them. They also thank Peter Scholze for pointing out a mistake in a previous version, and the anonymous referee for his/her careful reading which greatly improved the quality of the manuscript. The authors thank the University of Münster, Harvard University, Deutsche Forschungsgemeinschaft, the Institut de Mathématiques de Jussieu and the Technische Universität Darmstadt for financial and logistical support which made this research possible. Research of T.R. funded 
by the Deutsche Forschungsgemeinschaft (DFG, German Research Foundation) 394587809, Research of J.S. funded by DFG, Sonderforschungsbereich 878 and under Germany's Excellence Strategy EXC 2044-390685587, Mathematics Münster: Dynamics—Geometry-Structure.

\section{Conflict of Interest: None.}

\section{References}

[SGA73] D. M. Artin, A. Grothendieck and J. L. Verdier, Théorie des topos et cohomologie étale des schémas. Tome 3. Lecture Notes in Mathematics, 305. Springer, Berlin, 1973. Séminaire de Géométrie Algébrique du Bois-Marie 1963-1964 (SGA 4). Avec la collaboration de P. Deligne et B. Saint-Donat.

[Ayo07a] J. Ayoub, 'Les six opérations de Grothendieck et le formalisme des cycles évanescents dans le monde motivique. I', Astérisque 314 (2008), x+466 pp, 2007.

[Ayo07b] J. Ayoub, 'Les six opérations de Grothendieck et le formalisme des cycles évanescents dans le monde motivique. II', Astérisque (315) (2008), vi+364 pp, 2007.

[Ayo14] J. Ayoub, 'La réalisation étale et les opérations de Grothendieck', Annales Scientifiques de L'ens 47(1) (2014), 1-145. doi:10.24033/asens.2210.

[Bac19] T. Bachmann, 'Affine Grassmannians in $\mathbb{A}^{1}$-homotopy theory', Selecta Math. (N.S.) 25(2) (2019), Art. 25, 14. doi:10.1007/s00029-019-0471-1.

[BBD82] A. A. Beyllinson, J. Bernstein and P. Deligne, 'Faisceaux pervers', in Analysis and Topology on Singular Spaces, I (Luminy, 1981), Astérisque, 100 (Soc. Math. France, Paris, 1982), 5-171.

[BD99] A. Beilinson and V. Drinfeld, 'Quantization of Hitchin's integrable system and Hecke eigensheaves’.(1999). URL: http://www.math.utexas.edu/users/benzvi/Langlan ds.html.

[BK72] A. K. Bousfield and D. M. Kan, Homotopy Limits, Completions and Localizations, Lecture Notes in Mathematics, 304 (Springer, Berlin, 1972).

[BL16] M. V. Bondarko and A. Y. Luzgarev, 'On relative $K$-motives, weights for them, and negative $K$-groups'. Preprint, 2016, arXiv:1605.08435.

[Blo86] S. Bloch, 'Algebraic cycles and higher K-theory', Adv. Math. 61(3) (1986), 267-304.

[BLR90] S. Bosch, W. Lütkebohmert and M. Raynaud, Néron Models, Ergebnisse der Mathematik und ihrer Grenzgebiete (3) [Results in Mathematics and Related Areas (3)], 21 (Springer, Berlin, 1990), doi:10.1007/978-3-642-51438-8.

[BN19] U. Bunke and T. Nikolaus, 'Twisted differential cohomology', Algebr. Geom. Topol. 19(4) (2019), 1631-1710.

[Bon12] M. V. Bondarko, 'Weight structures and 'weights' on the hearts of $t$-structures', Homology, Homotopy Appl. 14(1) (2012), 239-261.

[Bon14] M. V. Bondarko, 'Weights for relative motives: relation with mixed complexes of sheaves', Int. Math. Res. Not. IMRN (17) (2014), 4715-4767.

[Bou68] N. Bourbaki, Éléments de mathématique. Fasc. XXXIV. Groupes et algèbres de Lie. Chapitre IV: Groupes de Coxeter et systèmes de Tits. Chapitre V: Groupes engendrés par des réflexions. Chapitre VI: systèmes de racines. Actualités Scientifiques et Industrielles, 1337. Hermann, Paris, 1968. 
[BS19] M. V. Bondarko and V. A. Sosnilo, 'On purely generated $\alpha$-smashing weight structures and weight-exact localizations', J. Algebra 535 (2019), 407-455.

[BT84] F. Bruhat and J. Tits, 'Groupes réductifs sur un corps local. II. Schémas en groupes. Existence d'une donnée radicielle valuée', Publ. Math. Inst. Hautes Études Sci. (60) (1984), 197-376. URL: http://www.numdam.org/item/PMIHES_1984_60_5_0/.

[CD09] D.-C. Cisinski and F. Déglise, 'Triangulated categories of mixed motives'. Preprint, 2009, arXiv:0912.2110.

[CD16] D.-C. Cisinski and F. Déglise, 'Étale motives', Compos. Math. 152(3) (2016), 556-666.

[Con14] B. Conrad, 'Reductive group schemes', in Autour des schémas en groupes, Vol. I, Panor. Synthèses, 42/43 (Soc. Math. France, Paris, 2014), 93-444.

[dCHL18] M. A. de Cataldo, T. J. Haines and L. Li, 'Frobenius semisimplicity for convolution morphisms', Math. Z. 289(1-2) (2018), 119-169.

[Dég08] F. Déglise, 'Around the Gysin triangle. II', Doc. Math. 13 (2008), 613-675.

[DG05] P. Deligne and A. B. Goncharov, 'Groupes fondamentaux motiviques de Tate mixte', Ann. Sci. Éc. Norm. Supér. (4) 38(1) (2005), 1-56.

[DI05] D. Dugger and D. C. Isaksen, 'Motivic cell structures', Algebr. Geom. Topol. 5 (2005), 615-652.

[DL76] P. Deligne and G. Lusztig, 'Representations of reductive groups over finite fields', Ann. of Math. (2) 103(1) (1976), 103-161.

[EG98] D. Edidin and W. Graham, 'Equivariant intersection theory', Invent. Math. 131(3) (1998), 595-634.

[EG15] M. Emerton and T. Gee, "'Scheme-theoretic images” of morphisms of stacks'. Preprint, 2015, arXiv:1506.06146.

[EK19] J. N. Eberhardt and S. Kelly, 'Mixed motives and geometric representation theory in equal characteristic', Selecta Math. (N.S.) 25(2) Art. 30, 54, 2019. doi:10.1007/s00029019-0475-x.

[Fal03] G. Faltings, 'Algebraic loop groups and moduli spaces of bundles', J. Eur. Math. Soc. (JEMS) 5(1) (2003), 41-68.

[Gai] D. Gaitsgory, 'Notes on geometric Langlands: Generalities on DG categories'. Preprint, available at the web site of the author.

[Gir71] J. Giraud, Cohomologie non abélienne, Die Grundlehren der mathematischen Wissenschaften, Band 179 (Springer, New York, 1971).

[GJ09] P. G. Goerss and J. F. Jardine, Simplicial Homotopy Theory, Modern Birkhäuser Classics (Birkhäuser Verlag, Basel, 2009), Reprint of the 1999 edition. doi:10.1007/978-3-03460189-4.

[GR17] D. Gaitsgory and N. Rozenblyum, 'A study in derived algebraic geometry', in Correspondences and Duality, Vol. I, Mathematical Surveys and Monographs, 221 (American Mathematical Society, Providence, RI, 2017).

[Gro61] A. Grothendieck, 'Éléments de géométrie algébrique: II. Étude globale élémentaire de quelques classes de morphismes', Publ. Math. Inst. Hautes Études Sci. (8) (1961), 222 URL: http://www.numdam.org/item/PMIHES_1961__8_5_0/.

[Hab11] S. Habibi, On the motive of a bundle, 2011. Thesis at the Universitá di Milano. URL: https://air.unimi.it/retrieve/handle/2434/212311/197090/phd_unimi_R08381.pdf.

[HR18a] T. J. Haines and T. Richarz, 'On the normality of Schubert varieties: remaining cases in positive characteristic'. Preprint, 2018, arXiv:1806.11001.

[HR18b] T. J. Haines and T. Richarz, 'The test function conjecture for local models of weilrestricted groups'. Preprint, 2018, arXiv:1805.07081. 
[HR18c] T. J. Haines and T. Richarz, 'The test function conjecture for parahoric local models'. Preprint, 2018. arXiv:1801.07094.

[Han95] M. Hanamura, 'Mixed motives and algebraic cycles. I', Math. Res. Lett. 2(6) (1995), 811-821.

[Hau15] R. Haugseng, 'Rectification of enriched $\infty$-categories', Algebr. Geom. Topol. 15(4) (2015), 1931-1982.

[Héb11] D. Hébert, 'Structure de poids à la Bondarko sur les motifs de Beilinson', Compos. Math. 147(5) (2011), 1447-1462.

[Hei10] J. Heinloth, 'Uniformization of $\mathcal{G}$-bundles', Math. Ann. 347(3) (2010), 499-528.

[Hei18] J. Heinloth, 'Langlands parameterization over function fields following V. Lafforgue', Acta Math. Vietnam 43(1) (2018), 45-66.

[HL18] V. Hoskins and S. P. Lehalleur, 'A formula for the Voevodsky motive of the moduli stack of vector bundles on a curve'. Preprint, 2018, arXiv:1809.02150.

[Hoy17] M. Hoyois, 'The six operations in equivariant motivic homotopy theory', Adv. Math. 305 (2017), 197-279. doi:10.1016/j.aim.2016.09.031.

[ILO12] L. Illusie, Y. Laszlo and F. Orgogozo, Travaux de Gabber sur l'uniformisation locale et la cohomologie étale des schémas quasi-excellents. Séminaire à l'École polytechnique 2006-2008. available at http://www.math.polytechnique.fr/ orgogozo/, 2012.

[Jan03] J. C. Jantzen, Representations of Algebraic Groups, 2nd edn, Mathematical Surveys and Monographs, 107 (American Mathematical Society, Providence, RI, 2003).

[Kha16] A. Khan, Motivic homotopy theory in derived algebraic geometry, 2016. PhD thesis, Universität Duisburg-Essen. URL: https://duepublico.uni-duisburg-essen.de/servlets/D erivateServlet/Derivate-42021/Diss_Khan.pdf.

[KW01] R. Kiehl and R. Weissauer, Weil Conjectures, Perverse Sheaves and l'adic Fourier Transform, Ergebnisse der Mathematik und ihrer Grenzgebiete. 3. Folge. A Series of Modern Surveys in Mathematics [Results in Mathematics and Related Areas. 3rd Series. A Series of Modern Surveys in Mathematics], 42 (Springer, Berlin, 2001), doi:10.1007/ 978-3-662-04576-3.

[KR03] R. Kottwitz and M. Rapoport, 'On the existence of F-crystals', Comment. Math. Helv. 78(1) (2003), 153-184.

[Laf18] V. Lafforgue, 'Chtoucas pour les groupes réductifs et paramétrisation de Langlands globale', J. Amer. Math. Soc. 31(3) (2018), 719-891.

[Lev16] B. Levin, 'Local models for Weil-restricted groups', Compos. Math. 152(12) (2016), 2563-2601.

[Lev93] M. Levine, 'Tate motives and the vanishing conjectures for algebraic $K$-theory', in Algebraic K-theory and Algebraic Topology (Lake Louise, AB, 1991), NATO Adv. Sci. Inst. Ser. C Math. Phys. Sci., 407 (Kluwer Academic Publishers, Dordrecht, 1993), 167-188.

[Lev98] M. Levine, Mixed Motives, Mathematical Surveys and Monographs, 57 (American Mathematical Society, Providence, RI, 1998).

[LO08] Y. Laszlo and M. Olsson, 'The six operations for sheaves on Artin stacks. II. Adic coefficients', Publ. Math. Inst. Hautes Études Sci. (107) (2008), 169-210, doi:10.1007/s10240-008-0012-5.

[LZ11] Y. Liu and W. Zheng, 'Enhanced six operations and base change theorem for higher Artin stacks'. Preprint, 2011, arXiv:1211.5948.

[LZ14] Y. Liu and W. Zheng, 'Enhanced adic formalism and perverse $t$-structures for higher Artin stacks'. Preprint, 2014, arXiv:1404.1128. 
[Lur09] J. Lurie, Higher Topos Theory, Annals of Mathematics Studies, 170 (Princeton University Press, Princeton, NJ, 2009).

[Lur17] J. Lurie, 'Higher Algebra'. 2017. URL: http://www.math.harvard.edu/ lurie/.

[MV07] I. Mirković and K. Vilonen, 'Geometric Langlands duality and representations of algebraic groups over commutative rings', Ann. of Math. (2) 166(1) (2007), 95-143.

[NSS15] T. Nikolaus, U. Schreiber and D. Stevenson, 'Principal $\infty$-bundles: general theory', J. Homotopy Relat. Struct. 10(4) (2015), 749-801.

[PR08] G. Pappas and M. Rapoport, 'Twisted loop groups and their affine flag varieties', Adv. Math. 219(1) (2008), 118-198. With an appendix by T. Haines and Rapoport.

[PZ13] G. Pappas and X. Zhu, 'Local models of Shimura varieties and a conjecture of Kottwitz', Invent. Math. 194(1) (2013), 147-254.

[Ras] S. Raskin, 'D-modules on infinite dimensional varieties'. Preprint, available at the web site of the author.

[SGA03] Revêtements étales et groupe fondamental (SGA 1). Documents Mathématiques (Paris) [Mathematical Documents (Paris)], 3. Société Mathématique de France, Paris, 2003. Séminaire de géométrie algébrique du Bois Marie 1960-61. [Algebraic Geometry Seminar of Bois Marie 1960-61], Directed by A. Grothendieck, With two papers by M. Raynaud, Updated and annotated reprint of the 1971 original [Lecture Notes in Mathematics, 224, Springer, Berlin; MR0354651 (50 \#7129)].

[Ric13] T. Richarz, 'Schubert varieties in twisted affine flag varieties and local models', J. Algebra 375 (2013), 121-147. doi:10.1016/j.jalgebra.2012.11.013.

[Ric16a] T. Richarz, 'Affine Grassmannians and geometric Satake equivalences', Int. Math. Res. Not. IMRN (12) (2016), 3717-3767. doi:10.1093/imrn/rnv226.

[Ric16b] T. Richarz, 'On the Iwahori Weyl group', Bull. Soc. Math. France 144(1) (2016), 117-124.

[RS19] T. Richarz and J. Scholbach, 'The motivic Satake isomorphism'. Preprint, 2019, arXiv: 1909.08322.

[Roz] N. Rozenblyum, Filtered colimits of $\infty$-categories. Harvard seminar notes.

[Ryd10] D. Rydh, 'Submersions and effective descent of étale morphisms', Bull. Soc. Math. France 138(2) (2010), 181-230.

[Sch17] P. Scholze, 'Etale cohomology of diamonds'. Preprint, 2017, arXiv:1709.07343.

[Soe00] W. Soergel, 'On the relation between intersection cohomology and representation theory in positive characteristic', J. Pure Appl. Algebra 152(1-3) (2000), 311-335. Commutative algebra, homological algebra and representation theory (Catania/Genoa/Rome, 1998).

[Sta17] The Stacks Project Authors. Stacks Project. http://stacks.math.columbia.edu, 2017.

[SVW18] W. Soergel, R. Virk and M. Wendt, 'Equivariant motives and geometric representation theory (with an appendix by F. Hörmann and M. Wendt)'. Preprint, 2018, arXiv: 1809.05480.

[SW13] P. Scholze and J. Weinstein, 'Moduli of p-divisible groups', Camb. J. Math. 1(2) (2013), 145-237.

[SW18] W. Soergel and M. Wendt, 'Perverse motives and graded derived category $\mathcal{O}$ ', J. Inst. Math. Jussieu 17(2) (2018), 347-395.

[Tit79] J. Tits, 'Reductive groups over local fields', in Automorphic Forms, Representations and L-functions (Proc. Sympos. Pure Math., Oregon State Univ., Corvallis, OR, 1977), Part 1, Proceedings of Symposia in Pure Mathematics, XXXIII (American Mathematical Society, Providence, RI, 1979), 29-69. 
[TV08] B. Toën and G. Vezzosi, 'Homotopical algebraic geometry. II. Geometric stacks and applications', Mem. Amer. Math. Soc. 193(902) (2008), x+224.

[Tot99] B. Totaro, 'The Chow ring of a classifying space', in Algebraic K-theory (Seattle, WA, 1997), Proceedings of Symposia in Pure Mathematics, 67 (American Mathematical Society, Providence, RI, 1999), 249-281. doi:10.1090/pspum/067/1743244.

[Tot16] B. Totaro, 'The motive of a classifying space', Geom. Topol. 20(4) (2016), 2079-2133.

[Var04] Y. Varshavsky, 'Moduli spaces of principal F-bundles', Sel. Math., New Ser. 10(1) (2004), 131-166.

[Voe00] V. Voevodsky, 'Triangulated categories of motives over a field', in Cycles, Transfers, and Motivic Homology Theories, Annals of Mathematics Studies, 143 (Princeton University Press, Princeton, NJ, 2000), 188-238.

[Wil08] J. Wildeshaus, 'Notes on Artin-Tate motives', in Autour des motifs, Vol. III, Panorama et Synthèses, 49 (2016), 101-131.

[Wil16] J. Wildeshaus, 'Pure motives, mixed motives and extensions of motives associated to singular surfaces', in Autour des motifs-École d'été Franco-Asiatique de Géométrie Algébrique et de Théorie des Nombres/Asian-French Summer School on Algebraic Geometry and Number Theory. Vol. III, Panoramas et Synthèses, 49 (Soc. Math. France, Paris, 2016), 65-100.

[Zhu17] X. Zhu, 'Affine Grassmannians and the geometric Satake in mixed characteristic', Ann. of Math. (2) 185(2) (2017), 403-492. 